\title{
BAR COMPLEXES AND EXTENSIONS OF CLASSICAL EXPONENTIAL FUNCTORS
}

\author{
ANTOINE TOUZÉ
}

\begin{abstract}
We compute Ext-groups between classical exponential functors (i.e. symmetric, exterior or divided powers) and their Frobenius twists. Our method relies on bar constructions, and bridges these Extgroups with the homology of Eilenberg-Mac Lane spaces.

Together with [T2], this article provides an alternative approach to classical Ext-computations [FS, [FFSS, C1, C2] in the category of strict polynomial functors over fields. We also obtain significant Extcomputations for strict polynomial functors over the integers.
\end{abstract}

\section{INTRODUCTION}

1.1. Given a commutative ring $\mathbb{k}$, we denote by $\mathcal{P}_{\mathbb{k}}$ the category of strict polynomial functors introduced by Friedlander and Suslin in [FS]. These strict polynomial functors are functors $F: \mathcal{V}_{\mathbb{k}} \rightarrow \mathbb{k}$-Mod from the category $\mathcal{V}_{\mathbb{k}}$ of finitely generated projective $\mathbb{k}$-modules to the category of $\mathbb{k}$-modules, equipped with an additional scheme-theoretic structure. The computation of Ext groups in $\mathcal{P}_{\mathbb{k}}$ has applications to the cohomology of algebraic groups and the Steenrod algebra, see $[\mathrm{P}]$.

The symmetric powers $S^{d}: V \mapsto S^{d}(V)$, the exterior powers $\Lambda^{d}: V \mapsto$ $\Lambda^{d}(V)$ or the divided powers $\Gamma^{d}: V \mapsto \Gamma^{d}(V)=\left(V^{\otimes d}\right)^{\mathfrak{S}_{d}}$ yield fundamental examples of strict polynomial functors. We refer to them as the 'classical exponential functors'. Indeed they are the most common graded functors $X^{*}$ satisfying an exponential formula, i.e. a graded isomorphism (see section 5 for more on exponential functors):

$$
X^{*}(V \oplus W) \simeq X^{*}(V) \otimes X^{*}(W) .
$$

If $\mathbb{k}$ is a field of positive characteristic $p$, we denote by $I^{(r)}$ the $r$-th Frobenius twist functor, for $r \in \mathbb{N}$, and by $F^{(r)}$ the precomposition of a strict polynomial functor $F$ by the $r$-th Frobenius twist functor (with the convention that $I^{(0)}$ is the identity functor, hence $F^{(0)}=F$ ). Frobenius twists are additive functors, so the twisted variant $X^{*(r)}$ of a classical exponential functor $X^{*}$ is also an exponential functor.

The classical exponential functors, and their twisted variants over a field $\mathbb{k}$ of positive characteristic, are among the most elementary examples of strict polynomial functors. However, the computation of extension groups in $\mathcal{P}_{\mathbb{k}}$ between these functors is a challenging problem, and the main topic of this article.

1991 Mathematics Subject Classification. 18G15, 57T30, 20G10.

The author was partially supported by the ANR HGRT (Projet BLAN08-2 338236). 
Let us first consider the untwisted case, over an arbitrary commutative ring $\mathbb{k}$. We order the classical exponential functors as follows: $\Gamma^{*}<\Lambda^{*}<S^{*}$. If $X^{*}$ and $Y^{*}$ are classical exponential functors with $X^{*} \leq Y^{*}$ then it is well-known 11 that $\operatorname{Ext}_{\mathcal{P}_{k}}^{i}\left(X^{*}, Y^{*}\right)$ is zero if $i>0$. The degree zero part is an elementary computation, so the only nontrivial computations to achieve are those of
(i) $\operatorname{Ext}_{\mathcal{P}_{\mathrm{k}}}^{*}\left(S^{*}, \Lambda^{*}\right)$,
(ii) $\operatorname{Ext}_{\mathcal{P}_{\mathrm{k}}}^{*}\left(\Lambda^{*}, \Gamma^{*}\right)$,
(iii) $\operatorname{Ext}_{\mathcal{P}_{\mathrm{k}}}^{*}\left(S^{*}, \Gamma^{*}\right)$.

Moreover, the first two extensions $(i)$ and (ii) are isomorphic by a duality argument. So we only consider the extension groups $(i)$ and (iii). Akin studied [A] the extension groups $(i)$. He actually performed computations in the equivalent category of modules over the Schur algebra. His results give a formula for the dimension of these Ext-groups when $\mathbb{k}$ is a field of positive characteristic, as well as the computation of $\operatorname{Ext}_{\mathcal{P}_{\mathbb{k}}}^{i}\left(S^{d}, \Lambda^{d}\right)$ for $i=1$ and $d-1$ when $\mathbb{k}$ is the ring of integers. The method of Akin uses the classical resolution of exterior powers by symmetric powers, given by the bar construction of the symmetric algebra.

Over a field $\mathbb{k}$ of positive characteristic $p$, we also consider the extensions between the classical exponential functors precomposed by Frobenius twists. Precomposition by Frobenius twists actually creates a lot of extensions, and the extension groups

$$
\operatorname{Ext}_{\mathcal{P}_{\mathrm{k}}}^{*}\left(X^{*(r)}, Y^{*(r)}\right)
$$

are non-trivial, even when the classical exponential functors $X^{*}$ is smaller than $Y^{*}$ (for the order defined before). The Ext-groups with $X^{*} \leq Y^{*}$ were computed in [FFSS]. In this article, the authors prove that these extension groups are equipped with a convolution product, as well as a coproduct of the same type and they compute these extension groups as trigraded bialgebras. Their method is different from the method of Akin, namely it relies on the analysis of some hypercohomology spectral sequences associated to Koszul and De Rham complexes. The remaining extension groups, i.e. those with $X^{*}>Y^{*}$, were computed in [C2] with the same techniques, together with a Koszul duality phenomenon. However, [C2, Thm 3.2], a key result in [C2], is false in characteristic 2 (as explained in remark 4.7).

1.2. The main goal of this article is to provide new methods to compute the extension groups in $\mathcal{P}_{\mathbb{k}}$ between classical exponential functors, and also between their twisted variants. Our methods differ from the methods used in [FFSS, C2. They rather take the same starting point as in Akin's computations [A], namely (iterated) bar complexes of the symmetric algebra. We obtain the following results.

(1) Over a field $\mathbb{k}$, our approach provides new independent computations of all the Ext-groups computed in FFSS, C2. The statements of our results are given in section 15.1. We carefully compare our results with the results given in [FFSS, C2] in section 15.3, In particular our computations agree with all the computations of [FFSS].

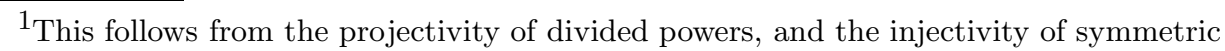
powers, except for the case of the extensions $\operatorname{Ext}_{\mathcal{P}_{\mathrm{k}}}^{i}\left(\Lambda^{*}, \Lambda^{*}\right)$. In the latter case, there are many proofs of this vanishing, and we will provide one in remark 7.6
} 
(2) Our methods also allow the computation of Ext-groups between classical exponential functors over the ring $\mathbb{k}=\mathbb{Z}$. In particular, we obtain in theorem 11.8 an explicit simple formula describing the abelian groups $\operatorname{Ext}_{\mathcal{P}_{\mathbb{Z}}}^{i}\left(S^{d}, \Lambda^{d}\right)$, for all $i$, thus extending the results of A]. Other (more complicated) descriptions are obtained in theorems 11.20 and 11.21).

To obtain our computations of extension groups in $\mathcal{P}_{\mathbb{k}}$ between (untwisted) classical exponential functors, we interpret them as the singular homology with $\mathbb{k}$ coefficients of some Eilenberg-Mac Lane spaces. The homology of these spaces was computed by Serre with $\mathbb{F}_{2}$ coefficients $[\mathrm{S}$, and by Cartan in general $\mathrm{Car}$. Then we elaborate on Cartan's results to obtain our Ext-computations. As an intermediate result, we obtain the following computation, of independent and general interest.

(3) We compute in theorem 10.14 the homology of iterated bar constructions of the divided power algebra $\Gamma^{*}(V)$, over any field $\mathbb{k}$. (See corollary 8.3 for the relation to Cartan's computations and remark 10.9 for an explanation why Cartan's computations only give the result over prime fields).

Apart from the results listed above, we feel that many technical results proved in the course of the article might be useful tools for further Extcomputations. For example, the key proposition 7.2 has a wider range of application than classical exponential functors.

1.3. We now briefly sketch the structure of our proof.

1.3.1. Structures on extension groups. Let $X^{*}$ and $Y^{*}$ be classical exponential functors, (or their Frobenius twisted variant if $\mathbb{k}$ is a field of positive characteristic). Before undertaking computations, we review in part 1 of the paper various structures which equip the extension groups $\operatorname{Ext}_{\mathcal{P}_{\mathbf{k}}}^{*}\left(X^{*}, Y^{*}\right)$, and determine which ones it is important to compute.

Functoriality. Parameterized extensions were introduced (for different reasons) in [C2 and [T2, and they are a key tool for our computations. If $V \in \mathcal{V}_{\mathbb{k}}$, we let $X^{* V}$ be the functor $\left.U \mapsto X^{*} \operatorname{Hom}_{\mathbb{k}}(V, U)\right)$. The parameterized extensions $\mathbb{E}(X, Y)$ are the (tri)graded strict polynomial functor defined by (the parameter is the variable ' $V$ ')

$$
\mathbb{E}(X, Y)(V)=\bigoplus_{i, d, e \geq 0} \mathbb{E}^{i}\left(X^{d}, Y^{e}\right)=\bigoplus_{i, d, e \geq 0} \operatorname{Ext}_{\mathcal{P}_{\mathrm{k}}}^{i}\left(X^{d V}, Y^{e}\right) .
$$

We denote by $\mathbb{H}(X, Y)$ the 'Hom-part', i.e. the summand with $i=0$, of $\mathbb{E}(X, Y)$. We will study the functors $\mathbb{E}(X, Y)$ rather than the mere extension groups $\operatorname{Ext}_{\mathcal{P}_{\mathbb{k}}}^{*}\left(X^{*}, Y^{*}\right)$ because functoriality reveals useful hidden structure (cf. the gradings and the coproducts below), and parameterized extension groups are not much more difficult to compute.

Gradings. The trigrading of $\mathbb{E}(X, Y)$ is artificial because the second and the third partial degrees are linearly dependent. For example, $\mathbb{E}^{*}\left(S^{d}, \Lambda^{e}\right)$ is zero if $d \neq e$, so we actually have to compute the bigraded extensions

$$
\mathbb{E}(S, \Lambda)=\bigoplus_{i, d \geq 0} \mathbb{E}^{i}\left(S^{d}, \Lambda^{d}\right)
$$


Moreover, since we study parameterized extension groups, even the bigrading is artificial. Indeed, the second grading (i.e. the exponent ' $d$ ') is encoded by the functoriality 2. Thus we only have to determine $\mathbb{E}(X, Y)$ as a strict polynomial functor, with a single Ext-grading.

Products. The parameterized extension groups $\mathbb{E}(X, Y)$ are equipped with a convolution product defined in [FFSS]. We say for short a ' $\mathcal{P}_{\mathbb{k}}$-graded algebra' for a graded strict polynomial functor endowed with an algebra structure. We will actually compute the $\mathcal{P}_{\mathbb{k}}$-graded algebra $\mathbb{E}(X, Y)$.

Coproducts. When the ground ring $\mathbb{k}$ is a field, $\mathbb{E}(X, Y)$ is also equipped with a coproduct defined in [FFSS]. The coproducts are computed in [FFSS, C2. However, we prove in section 5 that these parameterized extensions actually satisfy an exponential formula:

$$
\mathbb{E}(X, Y)(V \oplus W) \simeq \mathbb{E}(X, Y)(V) \otimes \mathbb{E}(X, Y)(W) .
$$

The coproduct is determined by the exponential formula, and the exponential formula is in turn determined by the product on $\mathbb{E}(X, Y)$. Thus, if we compute the $\mathcal{P}_{\mathbb{k}}$-graded algebra $\mathbb{E}(X, Y)$, we can easily deduce the coproduct from the product (see remark [5.9). That's why we limit ourselves to computing $\mathbb{E}(X, Y)$ as $\mathcal{P}_{\mathbb{k}}$-graded algebras in this article.

1.3.2. Extensions between classical exponential functors. As in the case of extension groups without parameters, the only non-trivial computations to achieve are the computations of $\mathbb{E}(S, \Lambda) \simeq \mathbb{E}(\Lambda, \Gamma)$ and $\mathbb{E}(S, \Gamma)$.

In part 2 of the paper, show that these extension groups can be computed (up to regrading) as the homology of the bar construction, resp. twice iterated bar construction, of the divided power algebra.

To prove this, we start in the same way as $[\mathrm{A}$, that is, we consider the resolutions of exterior and divided powers yield by iterated bar constructions of the symmetric algebra. Thus our extension groups may be computed (up to regrading) as the homology of the differential graded $\mathcal{P}_{\mathbb{k}}$-algebras $\mathbb{H}(S, \bar{B} S)$ and $\mathbb{H}\left(S, \bar{B}^{2} S\right)$. The regrading involves sign issues which are taken care of by the 'regrading functors' described in section 6. Then the key result is proposition 7.2, which says that in our situation (and many others), the symbols ' $\mathbb{H}$ ' and ' $\bar{B}$ ' can be interchanged. For example

$$
\mathbb{H}(S, \bar{B} S) \simeq \bar{B}(\mathbb{H}(S, S)) \simeq \bar{B} \Gamma .
$$

1.3.3. The homology of Eilenberg-Mac Lane spaces. So we are left with the problem of computing the $\mathcal{P}_{\mathbb{k}^{-}}$-graded algebra $H\left(\bar{B}^{n} \Gamma\right)$. Let $\Gamma\left(\mathbb{k}^{n}[2]\right)$ be the free divided power algebra over $\mathbb{k}$, generated by the $\mathbb{k}$-module $\mathbb{k}^{n}$ placed in degree 2 . We prove in corollary 8.3 an isomorphism of graded $\mathbb{k}$-algebras, natural with respect to $\mathbb{Z}^{m}$ :

$$
H_{*}\left(\bar{B}^{n}\left(\Gamma\left(\mathbb{Z}^{m} \otimes_{\mathbb{Z}} \mathbb{k}[2]\right)\right)\right) \simeq H_{*}^{\operatorname{sing}}\left(K\left(\mathbb{Z}^{m}, n+2\right), \mathbb{k}\right)
$$

between the homology of iterated bar constructions of divided powers, and the singular homology of Eilenberg-Mac Lane spaces. The later was computed by Cartan Car. However, Cartan's result is not enough for our purposes: we really need the left hand side as a $\mathcal{P}_{\mathbb{k}}$-graded algebra (see

\footnotetext{
${ }^{2}$ We call 'weight' the grading implicitly encoded by functoriality. See section 3 and definition 4.8
} 
more details on $\mathcal{P}_{\mathbb{k}}$-graded algebras in section [3). So we have to elaborate on Cartan's computations to get our results.

1.3.4. Frobenius twists. In part 4 of the paper, we explain how to retrieve the $\mathcal{P}_{\mathbb{k}}$-graded algebras $\mathbb{E}\left(X^{(r)}, Y^{(s)}\right)$ from $\mathcal{P}_{\mathbb{k}}$-graded algebra $\mathbb{E}(X, Y)$ in a simple way ( $\mathbb{k}$ is now a field of positive characteristic $p$ ). This part is a sequel to [T2] and some theorems of [T2] are actually used in section 13, while the other sections of part 4 are dedicated to complementary techniques.

1.4. Some conventions used in the article. In the article, we work over a commutative base ring $\mathbb{k}$, we denote by $\mathbb{k}-\bmod$ the category of $\mathbb{k}$-modules and by $\mathcal{V}_{\mathbb{k}}$ the full subcategory of finitely generated projective $\mathbb{k}$-modules. The $\mathbb{k}$-linear dual of a $\mathbb{k}$-module $V$ is $V^{\vee}=\operatorname{Hom}_{\mathbb{k}}(V, \mathbb{k})$. Otherwise explicitly stated, tensor products are taken over $\mathbb{k}$. In particular, if $V$ is a graded $\mathbb{k}$ module, the symbols $S(V), \Lambda(V)$ and $\Gamma(V)$ refer to the symmetric, the exterior and the divided power algebras over $\mathbb{k}$ (i.e. $S(V)=S_{\mathbb{k}}(V)$ ).

Otherwise explicitly stated, the word 'degree' means 'homological degree'. Homological degrees are denoted by indices, i.e. if $M$ is a complex, $M_{i}$ denotes the direct summand of degree $i$, and differentials lower the degree by one. Sometimes, cohomological degrees appear: they are denoted by exponents as in $\operatorname{Ext}_{\mathcal{P}_{\mathbb{k}}}^{i}(F, G)$. Such cohomological degrees can be converted into homological degrees by the usual formula: $M^{i}=M_{-i}$. If $M$ is an ungraded object, we denote by $M[i]$ the graded object with $M[i]_{j}=M$ if $i=j$ and zero if $i \neq j$. When cohomological degrees naturally appear in a formula, we use the notation $M\langle i\rangle$ to denote a copy of $M$ placed in cohomological degree $i$ (thus $M\langle i\rangle=M[-i]$ ).

It is well known that the abelian category $\mathcal{P}_{\mathbb{k}}$ of strict polynomial functors decomposes as a direct sum $\mathcal{P}_{\mathbb{k}}=\bigoplus_{d>0} \mathcal{P}_{d, \mathbb{k}}$. Elements of $\mathcal{P}_{d, \mathbb{k}}$ are called homogeneous functors, and if $F \in \mathcal{P}_{d, \mathbb{k}}$, the integer $d$ is often called the 'degree' of the functor [FS]. To avoid confusion with homological degrees, we do not use this terminology, we rather call $d$ the weight of the functor (cf section 2 below).

\section{Contents}

1. Introduction

Part 1. General structure results

2. Recollections of strict polynomial functors

3. A hierarchy of algebras

4. Structure of extension groups

5. The exponential property

Part 2. Extension groups between $S, \Lambda, \Gamma$, and bar constructions

6. Regrading functors

7. Bar constructions and extension groups

Part 3. Homology of Eilenberg-Mac Lane spaces

8. Bar constructions and Eilenberg-Mac Lane spaces 
9. Strict polynomial structures on divided power algebras

10. Explicit computations over a field

11. Explicit computations over the integers

\section{Part 4. Frobenius twists}

12. Décalages

13. The twisting spectral sequence and Troesch complexes

14. Splitting filtrations

15. Final results

References

\section{Part 1. General structure results}

In this part, we describe the framework and the notations for our computations.

\section{RECOLLECTIONS OF STRICT POLYNOMIAL FUNCTORS}

In this section, we briefly review basic facts of the theory of strict polynomial functors needed in the article. We refer the reader to $[\mathrm{FS}, \mathrm{SFB}, \mathrm{P}$, [11, K] for more details.

2.1. Strict polynomial functors. For $d \geq 0$, let $\Gamma^{d} \mathcal{V}_{\mathbb{k}}$ be the category with the same objects as $\mathcal{V}_{\mathbb{k}}$ (i.e. finitely generated projective $\mathbb{k}$-modules) but whose morphisms are $\mathfrak{S}_{d}$-equivariant maps:

$$
\operatorname{Hom}_{\Gamma^{d} \mathcal{V}_{\mathrm{k}}}(V, W)=\operatorname{Hom}_{\mathfrak{S}_{d}}\left(V^{\otimes d}, W^{\otimes d}\right),
$$

where $\mathfrak{S}_{d}$ acts on $V^{\otimes d}$ by permuting the factors of the tensor product, and composition in $\Gamma^{d} \mathcal{V}_{\mathbb{k}}$ is composition of $\mathfrak{S}_{d}$-equivariant maps (by convention, $V^{\otimes 0}=\mathbb{k}$, and $\left.\mathfrak{S}_{0}=\{1\}\right)$. The notation ' $\Gamma^{d} \mathcal{V}_{\mathbb{k}}$ ' is justified by the isomorphism $\Gamma^{d}\left(\operatorname{Hom}_{\mathbb{k}}(V, W)\right) \simeq \operatorname{Hom}_{\Gamma^{d} \mathcal{V}_{\mathbb{k}}}(V, W)$, induced by the isomorphism

$$
\operatorname{Hom}_{\mathfrak{S}_{d}}\left(V^{\otimes d}, W^{\otimes d}\right)=\operatorname{Hom}_{\mathbb{k}}\left(V^{\otimes d}, W^{\otimes d}\right)^{\mathfrak{S}_{d}} \simeq\left(\operatorname{Hom}_{\mathbb{k}}(V, W)^{\otimes d}\right)^{\mathfrak{S}_{d}} .
$$

The category $\mathcal{P}_{d, \mathbb{k}}$ of homogeneous strict polynomial functors of weight $d$ is the abelian category of $\mathbb{k}$-linear functors from $\Gamma^{d} \mathcal{V}_{\mathbb{k}}$ to $\mathbb{k}$-mod.

The abelian category $\mathcal{P}_{\mathbb{k}}$ of strict polynomial functors is the direct sum:

$$
\mathcal{P}_{\mathbb{k}}=\bigoplus_{d \geq 0} \mathcal{P}_{d, \mathbb{k}}
$$

So the weight is an implicit grading on strict polynomial functors. Each strict polynomial functor $F$ splits as a direct sum $F=\oplus F_{d}$ of homogeneous strict polynomial functors $F_{d}$ of weight $d$, and $\operatorname{Hom}_{\mathcal{P}_{\mathrm{k}}}\left(F_{d}, G_{e}\right)$ equals $\operatorname{Hom}_{\mathcal{P}_{d, \mathrm{k}}}\left(F_{d}, G_{e}\right)$ if $e=d$ and zero otherwise. 
2.2. Strict polynomial vs ordinary functors. Let $\mathcal{F}_{\mathbb{k}}$ denote the category of 'ordinary functors', i.e. functors from $\mathcal{V}_{\mathbb{k}}$ to $\mathbb{k}$-mod. There is a exact and faithful forgetful functor

$$
\mathcal{U}: \mathcal{P}_{\mathbb{k}} \rightarrow \mathcal{F}_{\mathbb{k}} .
$$

(The restriction $\mathcal{U}: \mathcal{P}_{d, \mathbb{k}} \rightarrow \mathcal{F}_{\mathbb{k}}$ is defined as the precomposition by the functor $\gamma_{d}: \mathcal{V}_{\mathbb{k}} \rightarrow \Gamma^{d} \mathcal{V}_{\mathbb{k}}$, where $\gamma_{d}$ is the identity on objects and $\gamma^{d}(f):=$ $f^{\otimes d}$.) Thus, we can think of strict polynomial functors as ordinary functors, equipped with a 'strict polynomial structure'. For example, the following functors

(i) the $d$-th tensor product $\otimes^{d}: V \mapsto V^{\otimes d}$,

(ii) the $d$-th exterior power $\Lambda^{d}: V \mapsto V^{\otimes d}$,

(iii) the $d$-th symmetric power $S^{d}: V \mapsto S^{d}(V)=\left(V^{\otimes d}\right)_{\mathfrak{S}_{d}}$ (coinvariants under the action of $\mathfrak{S}_{d}$ ),

(iv) the $d$-th divided power $\Gamma^{d}: V \mapsto \Gamma^{d}(V)=\left(V^{\otimes d}\right)^{\mathfrak{S}_{d}}$ (invariants under the action of $\mathfrak{S}_{d}$, the reason for the name 'divided power' is given in section (9).

have a canonical structure of homogeneous strict polynomial functors of weight $d$.

Remark 2.1. If $\mathbb{k}$ is an infinite field, the forgetful functor $\mathcal{U}: \mathcal{P}_{\mathbb{k}} \rightarrow \mathcal{F}_{\mathbb{k}}$ is full and faithful, so $\mathcal{P}_{\mathbb{k}}$ can be thought of as a full subcategory of $\mathcal{F}_{\mathbb{k}}$. In general, the behavior of the forgetful functor is more subtle. For example, if $\mathbb{k}=\mathbb{F}_{p}$, the $r$-th Frobenius twist functor $I^{(r)}$ [FS, (v) p.224] and the identity functor $I$ are nonisomorphic strict polynomial functors (they are homogeneous of different weights), but $\mathcal{U} I^{(r)}=\mathcal{U} I$. Even if we only consider homogeneous strict polynomial functors of a given weight, the restriction $\mathcal{U}: \mathcal{P}_{d, \mathbb{k}} \rightarrow \mathcal{F}_{\mathbb{k}}$ behaves badly. For example if $\mathbb{k}=\mathbb{F}_{2}$, one can show that $F=S^{2(1)} \otimes I^{(1)}$ and $G=S^{2} \otimes I^{(2)}$ are nonisomorphic objects of $\mathcal{P}_{6, \mathbb{F}_{2}}$, but $\mathcal{U}(F) \simeq \mathcal{U}(G)$.

2.3. Operations on strict polynomial functors. Usual operations on ordinary functors (composition, tensor products and duality) lift at the level of strict polynomial functors. If $F$ and $G$ are strict polynomial functors, their tensor product $(F \otimes G)(V)=F(V) \otimes G(V)$ is canonically equipped with the structure of a strict polynomial functor. If we restrict our attention to homogeneous functors, tensor product induces a functor:

$$
\otimes: \mathcal{P}_{d, \mathbb{k}} \times \mathcal{P}_{e, \mathbb{k}} \rightarrow \mathcal{P}_{d+e, \mathbb{k}}
$$

Given a strict polynomial functor $F$, the dual functor $F^{\sharp}: V \mapsto F\left(V^{\vee}\right)^{\vee}$ (where ${ }^{\vee}$ refers to the $\mathbb{k}$-linear dual) is canonically a strict polynomial functor. For example $S^{d \sharp} \simeq \Gamma^{d}, \Lambda^{d \sharp} \simeq \Lambda^{d}$ and $\Gamma^{d \sharp} \simeq S^{d}$. The functor

$$
\sharp: \mathcal{P}_{d, \mathbb{k}}^{\mathrm{op}} \rightarrow \mathcal{P}_{d, \mathbb{k}}
$$

is called 'Kuhn duality' in [FS]. If $\mathcal{P}_{d, \mathbb{k}}^{\prime}$ denotes the full subcategory of $\mathcal{P}_{d, \mathbb{k}}$ of functors with values in $\mathcal{V}_{\mathbb{k}}$, duality induces an equivalence of categories $\mathcal{P}_{d, \mathbb{k}}^{\prime \text { op }} \simeq \mathcal{P}_{d, \mathbb{k}}^{\prime}$.

Finally, the composition $G \circ F$ of a strict polynomial functor $G$ by a strict polynomial functor $F$ makes sense, provided $F$ takes finitely generated 
projective values. So composition yields a functor:

$$
\circ: \mathcal{P}_{d, \mathbb{k}} \times \mathcal{P}_{e, \mathbb{k}}^{\prime} \rightarrow \mathcal{P}_{d e, \mathbb{k}} .
$$

In particular, we can consider the precomposition by the following homogeneous functors. The functors $V \otimes: W \mapsto V \otimes W$, and $\operatorname{Hom}_{\mathbb{k}}(V,-): W \mapsto$ $\operatorname{Hom}_{\mathbb{k}}(V, W)$ (these functors have weight 1 ), and if $\mathbb{k}$ is a field of characteristic $p>0$, the $r$-th Frobenius twist $I^{(r)}$ (of weight $p^{r}$ ) [FS, (v) p.224]. Such compositions will occur frequently in the article.

Notation 2.2. For all $F \in \mathcal{P}_{\mathbb{k}}$, we use the following notations:

$$
F^{(r)}=F \circ I^{(r)}, \quad F_{V}:=F \circ(V \otimes), \quad F^{V}:=F \circ \operatorname{Hom}_{\mathbb{k}}(V,-) .
$$

The functors $F_{V}$ and $F^{V}$ are called 'parameterized functors' (the parameter is the finitely generated projective $\mathbb{k}$-module $V$ ).

2.4. Homological algebra in $\mathcal{P}_{\mathbb{k}}$. The abelian categories $\mathcal{P}_{d, \mathbb{k}}$, have enough projectives. To be more specific, a projective generator is given by the parameterized divided power

$$
\Gamma^{d, V}:=\operatorname{Hom}_{\Gamma^{d} \mathcal{V}_{\mathbb{k}}}(V,-) \simeq\left(\Gamma^{d}\right)^{V},
$$

for $V$ a free $\mathbb{k}$-module with rank greater or equal to $d$. The projectivity of $\Gamma^{d, V}$ (for all $V \in \mathcal{V}_{\mathbb{k}}$ ) is a consequence of the Yoneda isomorphism (frequently used in the sequel of the article):

$$
\operatorname{Hom}_{\mathcal{P}_{d, \mathrm{k}}}\left(\Gamma^{d, V}, F\right) \simeq F(V) .
$$

Since $\mathcal{P}_{\mathbb{k}}=\bigoplus_{d \geq 0} \mathcal{P}_{d, \mathbb{k}}$ the category $\mathcal{P}_{\mathbb{k}}$ also has enough projectives. If $F_{d}$ and $G_{e}$ are homogeneous strict polynomial functors of weight $d$, resp. $e$, then $\operatorname{Ext}_{\mathcal{P}_{\mathrm{k}}}^{*}\left(F_{d}, G_{e}\right)$ is equal to $\operatorname{Ext}_{\mathcal{P}_{d, \mathrm{k}}}^{*}\left(F_{d}, G_{e}\right)$ if $d=e$, and zero otherwise.

The following lemma explains that the parameterized symmetric powers $S_{V}^{d}$ play the role of injective functors, provided we restrict to functors with values in $\mathcal{V}_{\mathbb{k}}$ (which is the case for all the Ext-computations of the paper).

Lemma 2.3. Let $F, G \in \mathcal{P}_{d, \mathbb{k}}$. Assume that $F$ and $G$ take finitely generated projective values. Duality induces an isomorphism (natural in $F, G$ )

$$
\operatorname{Ext}_{\mathcal{P}_{\mathrm{k}}}^{*}(F, G) \simeq \operatorname{Ext}_{\mathcal{P}_{\mathrm{k}}}^{*}\left(G^{\sharp}, F^{\sharp}\right) .
$$

Moreover $G$ admits a coresolution by finite direct sums of copies of functors of the form $S_{V}^{d}$, and if $J_{G}$ is such a resolution, $\operatorname{Ext}_{\mathcal{P}_{\mathrm{k}}}^{*}(F, G)$ equals the homology of the complex $\operatorname{Hom}_{\mathcal{P}_{\mathrm{k}}}\left(F, J_{G}\right)$.

Proof. Let us denote by $\mathcal{P}_{\mathbb{k}}^{\prime}$ the full subcategory of $\mathcal{P}_{\mathbb{k}}$ whose objects are the strict polynomial functors with values in $\mathcal{V}_{\mathbb{k}}$. This is an exact subcategory of $\mathcal{P}_{\mathbb{k}}$, with projective objects $\Gamma^{d, V}$, cf. [SFB, Section 2]. A projective resolution in $\mathcal{P}_{\mathbb{k}}^{\prime}$ remains a projective resolution if we view it in $\mathcal{P}_{\mathbb{k}}$, so inclusion $\mathcal{P}_{\mathbb{k}}^{\prime} \hookrightarrow \mathcal{P}_{\mathbb{k}}$ induces an isomorphism at the level of Exts. 
The duality functor is an equivalence of categories when restricted to $\mathcal{P}_{\mathbb{k}}^{\prime}$. So the first assertion of lemma 2.3 follows from the commutative diagram:

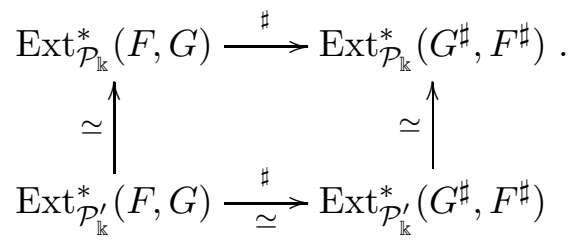

The second assertion of lemma 2.3 follows from the fact that category $\mathcal{P}_{\mathbb{k}}^{\prime}$ has enough injectives, and an injective cogenerator is provided by the functors $S_{V}^{d}$, cf. [SFB, Section 2].

\section{A hierarchy of AlgEBRAS}

In this article, the algebras considered are often endowed with an additional structure such as functoriality or an extra grading (called 'weight'). The categories of $\mathbb{k}$-algebras we will use are organized according to the following diagram.

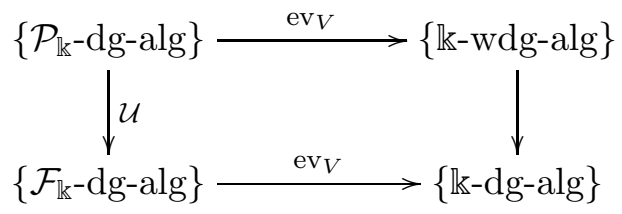

In this diagram, the arrows are functors induced by forgetting part of the structure. Thus, the category $\{\mathbb{k}-\mathrm{dg}-\mathrm{alg}\}$ on the bottom right corner of the diagram is the one having the less structured objects. In this section, we review the definitions of the various categories appearing in the hierarchy diagram $(\mathcal{H})$.

3.1. Differential graded algebras. By $\{\mathbb{k}-\mathrm{dg}$-alg $\}$, we denote the usual category of differential graded algebras over the commutative base ring $\mathbb{k}$.

If $A$ is a differential graded algebra, the degree of a homogeneous element $x \in A$ is denoted by $|x|$. Recall that the tensor product $A \otimes B$ of two dgalgebras has product $(a \otimes b) \cdot\left(a^{\prime} \otimes b^{\prime}\right)=(-1)^{\left|a^{\prime}\right||b|} a a^{\prime} \otimes b b^{\prime}$ and differential $\partial(a \otimes b)=(\partial a) \otimes b+(-1)^{|a|} a \otimes(\partial b)$.

Graded $\mathbb{k}$-algebras are viewed as dg-algebras with trivial differential, and ungraded $\mathbb{k}$-algebras are viewed as dg-algebras concentrated in degree zero.

3.2. Weighted differential graded algebras. By $\{\mathbb{k}-w d g-a l g\}$, we denote the category of weighted differential graded algebras over $\mathbb{k}$. The objects of this category are the weighted graded differential algebras over $\mathbb{k}$, that is, the dg-algebras $A$ equipped with an extra nonnegative grading, called 'weight'. The weight of a homogeneous element $x \in A$ is denoted by $w(x)$ and the weights are required to satisfy:

$$
w(x y)=w(x)+w(y), \quad w(\partial x)=w(x) .
$$

Morphisms of wdg-algebras are $\mathbb{k}$-linear maps which preserve the gradings and the weights, which are multiplicative and which commute with the differentials. 
The tensor product $A \otimes B$ of two wdg-algebras is defined by letting for all homogeneous elements $a, b$ :

(i) $|a \otimes b|=|a|+|b|$, and $w(a \otimes b)=w(a)+w(b)$,

(ii) $(a \otimes b) \cdot\left(a^{\prime} \otimes b^{\prime}\right)=(-1)^{\left|a^{\prime}\right||b|} a a^{\prime} \otimes b b^{\prime}$,

(iii) $\partial(a \otimes b)=(\partial a) \otimes b+(-1)^{|a|} a \otimes(\partial b)$.

Observe that the weights do not contribute to the signs in the definition of tensor products. Thus, forgetting the weights yields a functor, which preserves the tensor products:

$$
\{\mathbb{k}-\text { wdg-alg }\} \rightarrow\{\mathbb{k}-\text { dg-alg }\} .
$$

3.3. Functorial differential graded algebras. We denote by $\left\{\mathcal{F}_{\mathbb{k}}\right.$-dg-alg $\}$ the category of functorial differential graded algebras. The objects of this category are the functors $A: \mathcal{V}_{\mathbb{k}} \rightarrow\{\mathbb{k}$-dg-alg $\}$, and the morphisms are the natural transformations between such functors.

Let $V \in \mathcal{V}_{\mathbb{k}}$ be a finitely generated projective $\mathbb{k}$-module. Evaluation on $V$ yields a forgetful functor (we forget the naturality with respect to $V$ ):

$$
\left\{\mathcal{F}_{\mathbb{k}}-\mathrm{dg}-\mathrm{alg}\right\} \stackrel{\mathrm{ev}_{V}}{\longrightarrow}\{\mathbb{k}-\mathrm{dg}-\mathrm{alg}\} .
$$

The tensor product of two $\mathcal{F}_{\mathbb{k}}$-dg-algebras is defined in the target category, so that the forgetful functor commutes with tensor products.

3.4. Strict polynomial differential graded algebras. We denote by $\left\{\mathcal{P}_{\mathbb{k}}\right.$-dg-alg $\}$ the category of strict polynomial differential graded algebras. This is a category of highly structured algebras. To be more specific, we make the following definitions.

Definition 3.1. Let $\mathbb{k}$ be a commutative ring. A strict polynomial differential graded algebra ( $\mathcal{P}_{\mathbb{k}}$-dg-algebra) $A$ is a collection of strict polynomial functors $\left\{A_{i, d}\right\}$ for $i \in \mathbb{Z}$ and $d \geq 0$, equipped with morphisms of strict polynomial functors $\eta: \mathbb{k} \rightarrow A_{0,0}, m: A_{i, d} \otimes A_{j, e} \rightarrow A_{i+j, d+e}$, and $\partial: A_{i, d} \rightarrow A_{i-1, d}$ satisfying the following properties.

(i) For all $d \geq 0$, the strict polynomial functor $A_{i, d}$ is homogeneous of weight $d$.

(ii) For all $V \in \mathcal{V}_{\mathbb{k}}$, the unit $\eta$, the multiplication $m$ and the differential $\partial$ make the $\mathbb{k}$-module $A(V)=\bigoplus_{i, d} A_{i, d}(V)$ into a weighted differential graded algebra.

The functor $A_{i, d}$ will be referred to as the homogeneous part of degree $i$ and weight $d$ of $A$.

The $\mathcal{P}_{\mathbb{k}}$-graded algebras are the $\mathcal{P}_{\mathbb{k}}$-dg-algebras with trivial differential, and the $\mathcal{P}_{\mathbb{k}}$-algebras are the $\mathcal{P}_{\mathbb{k}}$-dg-algebras concentrated in degree zero.

Symmetric, exterior and divided powers yield fundamental examples of $\mathcal{P}_{\mathbb{k}}$-graded algebras. To be more specific, let $X=S, \Lambda$ or $\Gamma$. Then $\operatorname{Hom}_{\mathcal{P}_{\mathbb{k}}}\left(X^{d} \otimes X^{e}, X^{d+e}\right)$ is a rank one free $\mathbb{k}$-module. If $X=S$ or $\Lambda$, it is generated by the unique morphism fitting into diagram $(D 1)$ below. For $X=\Gamma$, it is generated by the unique morphism fitting in diagram $(D 2)$, where $\mathrm{S}(d, e) \subset \mathfrak{S}_{d+e}$ is the set of all $(d, e)$-shuffles. We call this generator 
the canonical map between $X^{d} \otimes X^{e}$ and $X^{d+e}$.
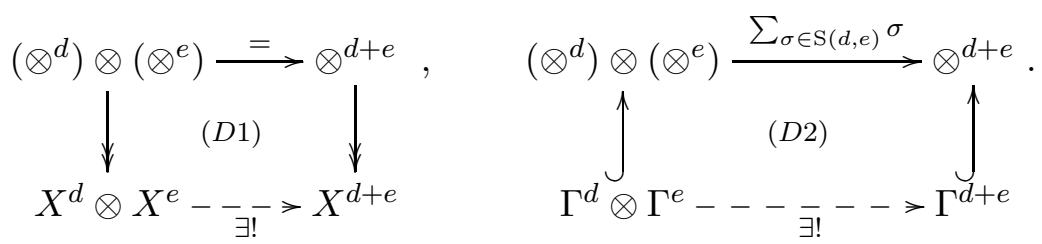

In the remainder of the article, we will mostly use the $\mathcal{P}_{\mathbb{k}}$-graded algebras of the following type.

- We denote by $S[i]$ the symmetric algebra over generators of homological degree $i$. That is, $S[i]$ is the strict polynomial graded algebra whose homogeneous part of degree $d i$ and weight $d$ equals $S^{d}$, with multiplication defined by the canonical maps $S^{d} \otimes S^{e} \rightarrow S^{d+e}$. The algebra $S[0]$ will simply be denoted by $S$. We define $\Lambda[i]$ and $\Gamma[i]$ (and $\Lambda, \Gamma$ ) similarly.

- More generally, if $F$ is a graded strict polynomial functor with values in $\mathcal{V}_{\mathbb{k}}$, we denote by $S(F)$ the symmetric algebra on $F$. It is formed by the graded strict polynomial functors $S^{d} \circ F$ (with degrees defined as usual), and the multiplication is given by evaluating the canonical map $S^{d} \otimes S^{e} \rightarrow S^{d+e}$ on $F$. We define the exterior algebra $\Lambda(F)$ over $F$, and the divided power algebra $\Gamma(F)$ over $F$ similarly.

Definition 3.2. A morphism of $\mathcal{P}_{\mathbb{k}_{\mathrm{k}}}$-dg-algebras $f: A \rightarrow B$ is a collection of morphisms of strict polynomial functors $f_{i, d}: A_{i, d} \rightarrow B_{i, d}$ which commute with the multiplications and the differentials of $A$ and $B$.

We define the tensor product $A \otimes B$ of two $\mathcal{P}_{\mathbb{k}^{-}}$-dg-algebras by formulas similar to the ones for the wdg-algebras (i.e. the degrees introduce Koszul signs in the definition of the product and the differentials, but the weights do not introduce such Koszul signs). The forgetful functor $\mathcal{U}: \mathcal{P}_{\mathbb{k}} \rightarrow \mathcal{F}_{\mathbb{k}}$ induces a functor:

$$
\left\{\mathcal{P}_{\mathbb{k}} \text {-dg-alg }\right\} \stackrel{\mathcal{U}}{\rightarrow}\left\{\mathcal{F}_{\mathbb{k}} \text {-dg-alg }\right\}
$$

which commutes with tensor products. If $V \in \mathcal{V}_{\mathbb{k}}$ is a finitely generated projective $\mathbb{k}$-module, evaluation on $V$ induces a forgetful functor:

$$
\left\{\mathcal{P}_{\mathbb{k}}-\text { dg-alg }\right\} \stackrel{\text { ev }_{V}}{\longrightarrow}\{\mathbb{k} \text {-wdg-alg }\},
$$

which also commutes with tensor products.

Remark 3.3. Let $A$ be a $\mathcal{P}_{\mathbb{k}}$-dg-algebra. Then knowing the collection of wdg-algebras $A(V), V \in \mathcal{V}_{\mathbb{k}}$, and the $\mathcal{F}_{\mathbb{k}}$-dg-algebra $\mathcal{U} A$ is usually not sufficient to determine $A$ as a $\mathcal{P}_{\mathbb{k}}$-dg-algebra. Indeed, one can find (see remark 2.1) nonisomorphic functors $F, G \in \mathcal{P}_{d, \mathbb{k}}$, such that $\mathcal{U}(F) \simeq \mathcal{U}(G)$. Then the symmetric algebras $A=S(F)$ and $B=S(G)$ are not isomorphic as $\mathcal{P}_{\mathbb{k}}$-algebras, although we have isomorphisms of $\mathcal{F}_{\mathbb{k}}$-algebras $\mathcal{U} A=\mathcal{U} B$, and isomorphisms of wg-algebras $A(V) \simeq B(V)$ for all $V$.

3.5. More strict polynomial objects. In the article, we also need other strict polynomial objects. For instance, the strict polynomial commutative differential graded algebras ( $\mathcal{P}_{\mathbb{k}^{\mathrm{k}}}$-cdg-algebras) are the cdg-algebras which are commutative as differential graded algebras, i.e. $x y=(-1)^{|x||y|} y x$ for all 
homogeneous elements $x, y \in A(V)$, for all $V \in \mathcal{V}_{\mathbb{k}}$. The algebras can also be augmented $\left(\mathcal{P}_{\mathbb{k}}\right.$-dga-algebras), or commutative and augmented $\left(\mathcal{P}_{\mathbb{k}^{-}}-\right.$ cdga-algebras). We also use strict polynomial differential graded coalgebras $\left(\mathcal{P}_{\mathbb{k}}\right.$-dg-coalgebras), etc. It is quite obvious how to adapt definition 3.1 to these cases, and we leave this to the reader.

\section{Structure of extension Groups}

4.1. Parameterized extension groups. In this section we introduce the parameterized extension groups $\mathbb{E}(F, G)$ alluded to in the introduction of the paper. We begin by parameterized Hom-groups $\mathbb{H}(F, G)=\mathbb{E}^{0}(F, G)$. Let $F \in \mathcal{P}_{d, \mathbb{k}}$, and recall from notation 2.2 the parameterized functors:

$$
F_{V}: W \mapsto F(V \otimes W) \quad \text { and } \quad F^{V}: W \mapsto F\left(\operatorname{Hom}_{\mathbb{k}}(V, W)\right) .
$$

Morphisms $f \in \operatorname{Hom}_{\Gamma^{d} \mathcal{V}_{\mathrm{k}}}\left(V, V^{\prime}\right)$ induce morphisms of strict polynomial functors $F_{V} \rightarrow F_{V^{\prime}}$ and $F^{V^{\prime}} \rightarrow F^{V}$. Hence, for all $F, G \in \mathcal{P}_{d, \mathbb{k}}$ we have functors:

$$
\begin{aligned}
\Gamma^{d} \mathcal{V}_{\mathbb{k}} & \rightarrow & \mathbb{k}-\bmod & \Gamma^{d} \mathcal{V}_{\mathrm{k}} & \rightarrow & \mathbb{k}-\bmod \\
V & \mapsto & \operatorname{Hom}_{\mathcal{P}_{d, \mathbb{k}}}\left(F^{V}, G\right) & V & \mapsto & \operatorname{Hom}_{\mathcal{P}_{d, \mathbb{k}}}\left(F, G_{V}\right)
\end{aligned} .
$$

Lemma 4.1. The two strict polynomial functors

$$
V \mapsto \operatorname{Hom}_{\mathcal{P}_{d, \mathrm{k}}}\left(F^{V}, G\right) \quad \text { and } \quad V \mapsto \operatorname{Hom}_{\mathcal{P}_{d, \mathrm{k}}}\left(F, G_{V}\right)
$$

are canonically isomorphic. We denote them by $\mathbb{H}(F, G)$.

Proof. Let us first take $F=\Gamma^{d, U}$. Then $\left(\Gamma^{d, U}\right)^{V}=\Gamma^{d, U \otimes V}$ and the Yoneda lemma yields an isomorphism:

$$
\operatorname{Hom}_{\mathcal{P}_{d, \mathrm{k}}}\left(\left(\Gamma^{d, U}\right)^{V}, G\right) \simeq G(U \otimes V) \simeq \operatorname{Hom}_{\mathcal{P}_{d, \mathrm{k}}}\left(\Gamma^{d, U}, G_{V}\right),
$$

natural with respect to $G$, to $f \in \operatorname{Hom}_{\Gamma^{d} \mathcal{V}_{\mathrm{k}}}\left(V, V^{\prime}\right)$ and $g \in \operatorname{Hom}_{\Gamma^{d} \mathcal{V}_{\mathbb{k}}}\left(U, U^{\prime}\right)$ (or equivalently to $g \in \operatorname{Hom}_{\mathcal{P}_{d, \mathbb{k}}}\left(\Gamma^{d, U^{\prime}}, \Gamma^{d, U}\right)$ ). Since the $\Gamma^{d, U}, U \in \mathcal{V}_{\mathbb{k}}$, form a projective generator of $\mathcal{P}_{d, \mathbb{k}}$, we can take presentations of $F$ to extend this isomorphism to all $F \in \mathcal{P}_{d, \mathbb{k}}$.

Let us turn to the category $\mathcal{P}_{\mathbb{k}}$ of strict polynomial functors. If $F, G$ are strict polynomial functors, they split as finite direct sums $F=\bigoplus F_{d}$ and $G=\bigoplus G_{d}$, where $F_{d}$ and $G_{d}$ are homogeneous of weight $d$, and by lemma 4.1, the functors

$$
\begin{aligned}
& V \mapsto \operatorname{Hom}_{\mathcal{P}_{\mathrm{k}}}\left(F^{V}, G\right)=\bigoplus \operatorname{Hom}_{\mathcal{P}_{d, \mathrm{k}}}\left(F_{d}^{V}, G_{d}\right) \\
& V \mapsto \operatorname{Hom}_{\mathcal{P}_{\mathrm{k}}}\left(F, G_{V}\right)=\bigoplus \operatorname{Hom}_{\mathcal{P}_{d, \mathrm{k}}}\left(F_{d},\left(G_{d}\right)_{V}\right)
\end{aligned}
$$

are canonically isomorphic strict polynomial functors, which we still denote by $\mathbb{H}(F, G)$. The following lemma summarizes the main properties of parameterized Hom groups.

Lemma 4.2. Let $\mathbb{k}$ be a commutative ring. Parameterized Hom groups yield a bifunctor:

$$
\begin{array}{clc}
\mathcal{P}_{\mathbb{k}}^{\mathrm{op}} \times \mathcal{P}_{\mathbb{k}} & \rightarrow & \mathcal{P}_{\mathbb{k}} \\
(F, G) & \mapsto & \mathbb{H}(F, G)
\end{array} .
$$

If $F, G$ are homogeneous of weight $d$, then so is $\mathbb{H}(F, G)$. Moreover:

(1) If $F, G$ take values in $\mathcal{V}_{\mathbb{k}}$, Kuhn duality yields an isomorphism of strict polynomial functors $\mathbb{H}(F, G) \simeq \mathbb{H}\left(G^{\sharp}, F^{\sharp}\right)$, natural in $F, G$. 
(2) If $G \in \mathcal{P}_{d, \mathbb{k}}$, there is an isomorphism $\mathbb{H}\left(\Gamma^{d}, G\right) \simeq G$, natural in $G$.

(3) Tensor products induce morphisms of strict polynomial functors:

$$
\mathbb{H}(F, G) \otimes \mathbb{H}\left(F^{\prime}, G^{\prime}\right) \stackrel{\otimes}{\rightarrow} \mathbb{H}\left(F \otimes F^{\prime}, G \otimes G^{\prime}\right) .
$$

Proof. The first part of lemma 4.2 follows from lemma 4.1. To prove (1), we can assume that $F, G$ are homogeneous of weight $d$. Since $\left(F^{V}\right)^{\sharp}=\left(F^{\sharp}\right)_{V}$, there is an isomorphism natural in $F, G$ and $f \in \operatorname{Hom}_{\Gamma^{d} \mathcal{V}_{\mathrm{k}}}\left(V, V^{\prime}\right)$

$$
\operatorname{Hom}_{\mathcal{P}_{d, \mathrm{k}}}\left(F^{V}, G\right) \simeq \operatorname{Hom}_{\mathcal{P}_{d, \mathrm{k}}}\left(G^{\sharp},\left(F^{V}\right)^{\sharp}\right)=\operatorname{Hom}_{\mathcal{P}_{d, \mathrm{k}}}\left(G^{\sharp},\left(F^{\sharp}\right)_{V}\right) .
$$

Whence the result. (2) is the Yoneda lemma. For (3), we can assume that $F, G$ (resp. $\left.F^{\prime}, G^{\prime}\right)$ are homogeneous of degree $d$ (resp. e). The map:

$$
\operatorname{Hom}_{\mathcal{P}_{d, \mathrm{k}}}\left(F^{V}, G\right) \otimes \operatorname{Hom}_{\mathcal{P}_{e, \mathrm{k}}}\left(\left(F^{\prime}\right)^{W}, G^{\prime}\right) \stackrel{\otimes}{\rightarrow} \operatorname{Hom}_{\mathcal{P}_{d+e, \mathrm{k}}}\left(F^{V} \otimes\left(F^{\prime}\right)^{W}, G \otimes G^{\prime}\right)
$$

is natural with respect to $f \in \operatorname{Hom}_{\Gamma^{d} \mathcal{V}_{\mathbf{k}}}\left(V, V^{\prime}\right)$ and $g \in \operatorname{Hom}_{\Gamma^{e}} \mathcal{V}_{\mathbf{k}}\left(W, W^{\prime}\right)$ (i.e. it is a morphism of strict polynomial bifunctors). Hence it becomes a morphism of strict polynomial functors if one takes $V=W$.

To define parameterized extension groups, we fix for each $F$ a projective resolution $P_{F}$ in $\mathcal{P}_{\mathbb{k}}$. We let $\mathbb{E}(F, G)$ be the homology of the complex of strict polynomial functors $\mathbb{H}\left(P_{F}, G\right)$. The following proposition follows directly from lemma 4.2.

Proposition 4.3. Let $\mathbb{k}$ be a commutative ring and let $F, G$ be strict polynomial functors over $\mathbb{k}$. For all $i \geq 0$, the functors

$$
V \mapsto \operatorname{Ext}_{\mathcal{P}_{\mathbb{k}}}^{i}\left(F^{V}, G\right) \quad \text { and } \quad V \mapsto \operatorname{Ext}_{\mathcal{P}_{\mathbf{k}}}^{i}\left(F, G_{V}\right)
$$

are isomorphic strict polynomial functors. We denote them by $\mathbb{E}^{i}(F, G)$. This yields bifunctors:

$$
\begin{array}{clc}
\mathcal{P}_{\mathbb{k}}^{\mathrm{op}} \times \mathcal{P}_{\mathbb{k}} & \rightarrow & \mathcal{P}_{\mathbb{k}} \\
(F, G) & \mapsto & \mathbb{E}^{i}(F, G)
\end{array} .
$$

The homogeneous part of weight $d$ of the strict polynomial functor $\mathbb{E}^{i}(F, G)$ is $\mathbb{E}^{i}\left(F_{d}, G_{d}\right)$, where $F_{d}$ and $G_{d}$ denote the homogeneous parts of $F$ and $G$ of weight d. Moreover, if $F, G$ take values in $\mathcal{V}_{\mathbb{k}}$, Kuhn duality induces an isomorphism $\mathbb{E}^{i}(F, G) \simeq \mathbb{E}^{i}\left(G^{\sharp}, F^{\sharp}\right)$. Finally, tensor products induce morphisms of strict polynomial functors

$$
\mathbb{E}^{i}(F, G) \otimes \mathbb{E}^{j}\left(F^{\prime}, G^{\prime}\right) \rightarrow \mathbb{E}^{i+j}\left(F \otimes F^{\prime}, G \otimes G^{\prime}\right) .
$$

4.2. Convolution products. We now introduce the convolution product ' $\star$ ' on the parameterized Hom-groups between a $\mathcal{P}_{\mathbb{k}}$-coalgebra $C$ and a $\mathcal{P}_{\mathbb{k}}$ dg-algebra $A$. This convolution product can be defined more generally when $C$ is a differential graded object, see e.g. [LV, Chap. 2], but we shall only need the case when $C$ is concentrated in degree zero. So we only describe the latter case, where the signs are slightly simpler.

Definition 4.4. Let $C$ be a $\mathcal{P}_{\mathbb{k}^{\mathrm{k}}}$-coalgebra and let $A$ be a $\mathcal{P}_{\mathbb{k}^{\mathrm{k}}}$-dg-algebra. We denote by $\mathbb{H}(C, A)$ the $\mathcal{P}_{\mathbb{k}}$-dg-algebra defined as follows.

(i) The homogeneous part of $\mathbb{H}(C, A)$ of degree $i$ and weight $d$ equals $\mathbb{H}\left(C_{0, d}, A_{i, d}\right)$. 
(ii) The differential $\partial^{\prime}$ of $\mathbb{H}(C, A)$ is given by postcomposing by the differential of $A$ :

$$
\partial^{\prime}:=\mathbb{H}\left(C_{0, d}, \partial\right): \mathbb{H}\left(C_{0, d}, A_{i, d}\right) \rightarrow \mathbb{H}\left(C_{0, d}, A_{i-1, d}\right) .
$$

(iii) The convolution product $\star$ is defined as the composite:

$$
\begin{aligned}
\mathbb{H}\left(C_{0, d}, A_{i, d}\right) \otimes \mathbb{H}\left(C_{0, e}, A_{j, e}\right) \stackrel{\otimes}{\rightarrow} \mathbb{H}\left(C_{0, d} \otimes C_{0, d}, A_{i, e} \otimes A_{j, e}\right) \\
\stackrel{\mathbb{H}(\Delta, m)}{\longrightarrow} \mathbb{H}\left(C_{0, d+e}, A_{i+j, d+e}\right) .
\end{aligned}
$$

(iv) The unit $\eta^{\prime}$ is induced by the unit of $A$ and the counit of $C$ :

$$
\eta^{\prime}:=\mathbb{H}(\epsilon, \eta): \mathbb{k}=\mathbb{H}(\mathbb{k}, \mathbb{k}) \rightarrow \mathbb{H}\left(C_{0,0}, A_{0,0}\right) .
$$

If $C$ is coaugmented (with coaugmentation $\eta: \mathbb{k} \rightarrow C$ ) and $A$ is augmented (with augmentation $\epsilon: A \rightarrow \mathbb{k}$ ), the convolution algebra $\mathbb{H}(C, A)$ is augmented, with augmentation $\mathbb{H}(\eta, \epsilon): \mathbb{H}(C, A) \rightarrow \mathbb{H}(\mathbb{k}, \mathbb{k})=\mathbb{k}$. Thus, convolution algebras yield a functor:

$$
\mathbb{H}(C,-):\left\{\mathcal{P}_{\mathbb{K}^{\mathbb{2}}} \text {-dga-alg }\right\} \rightarrow\left\{\mathcal{P}_{\mathbb{k}} \text {-dga-alg }\right\} .
$$

The following lemma is an easy check.

Lemma 4.5. Assume that $C$ is a commutative $\mathcal{P}_{\mathbb{k}}$-coalgebra, and that $A$ is a graded commutative $\mathcal{P}_{\mathbb{k}}$-dg-algebra. Then $\mathbb{H}(C, A)$ is graded commutative.

We have the following elementary computations of convolution algebras.

Lemma 4.6. Let $\mathbb{k}$ be a commutative ring. Let $C$ be a $\mathcal{P}_{\mathbb{k}}$-coalgebra, and let $A$ be a $\mathcal{P}_{\mathbb{k}}$-algebras. The following isomorphisms of $\mathcal{P}_{\mathbb{k}}$-algebras hold.

$$
\mathbb{H}(\Gamma, A) \simeq A, \quad \mathbb{H}(C, S) \simeq C^{\sharp}, \quad \mathbb{H}(S, \Gamma) \simeq \Gamma .
$$

Moreover, $\mathbb{H}(S, \Lambda) \simeq \mathbb{H}(\Lambda, \Gamma)$ is isomorphic to $\Gamma$ if $2=0$ in $\mathbb{k}$, and to $\Lambda$ otherwise.

Proof. We shall only prove the case of $\mathbb{H}(\Lambda, \Gamma)$. First, we have:

$$
\mathbb{H}\left(\Lambda^{d}, \otimes^{d}\right)(V) \simeq \operatorname{Hom}_{\mathcal{P}_{\mathfrak{k}}}\left(\Lambda^{d}, \otimes_{V}^{d}\right) \simeq \operatorname{Hom}_{\mathcal{P}_{\mathrm{k}}}\left(\Lambda^{d}, \otimes^{d}\right) \otimes V^{\otimes d} .
$$

The $\mathbb{k}$-module $\operatorname{Hom}_{\mathcal{P}_{\mathbb{k}}}\left(\Lambda^{d}, \otimes^{d}\right)$ is free of rank one, with generator the antisymmetrization map $\Delta_{d}: \Lambda^{d} \rightarrow \otimes^{d}$. So the isomorphism above can be rewritten as an isomorphism of functors

$$
\mathbb{H}\left(\Lambda^{d}, \otimes^{d}\right) \simeq \otimes^{d} .
$$

For all $\sigma \in \mathfrak{S}_{d}$, we identify $\sigma$ with the morphism $\otimes^{d} \rightarrow \otimes^{d}$ which permutes the factors of the tensor product. Since $\sigma \circ \Delta_{d}=\epsilon(\sigma) \Delta_{d}$, the $\mathfrak{S}_{d}$-module $\operatorname{Hom}_{\mathcal{P}_{\mathbb{k}}}\left(\Lambda^{d}, \otimes^{d}\right)$, where $\sigma$ acts as $\operatorname{Hom}\left(\Lambda^{d}, \sigma\right)$, is isomorphic to $\mathbb{k}$ with action of $\mathfrak{S}_{d}$ given by the signature. So the isomorphism $(*)$ is $\mathfrak{S}_{d}$-equivariant if $\sigma$ acts as $\mathbb{H}\left(\Lambda^{d}, \sigma\right)$ on the left hand side, and as $\epsilon(\sigma) \sigma$ on the right hand side.

Now, $\Gamma^{d}$ (resp. $\Lambda^{d}$ if $2 \neq 0$ in $\mathbb{k}$ ) is the intersection of the kernels of the maps Id $-\sigma: \otimes^{d} \rightarrow \otimes^{d}$ (resp. Id $\left.-\epsilon(\sigma) \sigma\right)$, for $\sigma \in \mathfrak{S}_{d}$. So by left exactness of parameterized Homs we obtain that $\mathbb{H}\left(\Lambda^{d}, \Gamma^{d}\right)$ is isomorphic to $\Lambda^{d}$ if $2 \neq 0$ in $\mathbb{k}$ and to $\Gamma^{d}$ if $2=0$ in $\mathbb{k}$.

It remains to identify the products. Let $\Delta_{d, e}: \Lambda^{d+e} \rightarrow \Lambda^{d} \otimes \Lambda^{e}$ denote the comultiplication of $\Lambda$. Since $\left(\Delta_{d} \otimes \Delta_{e}\right) \circ \Delta_{d, e}=\Delta_{d+e}$, the composite

$$
\mathbb{H}\left(\Lambda^{d}, \otimes^{d}\right) \otimes \mathbb{H}\left(\Lambda^{e}, \otimes^{e}\right) \stackrel{\otimes}{\rightarrow} \mathbb{H}\left(\Lambda^{d} \otimes \Lambda^{e}, \otimes^{d+e}\right) \rightarrow \mathbb{H}\left(\Lambda^{d+e}, \otimes^{d+e}\right)
$$


identifies with the identity map $\left(\otimes^{d}\right) \otimes\left(\otimes^{e}\right) \rightarrow \otimes^{d+e}$. We identify the product on $\mathbb{H}\left(\Lambda^{d}, \Gamma^{d}\right)$ by viewing it as a subalgebra of the signed shuffle algebra, with $\otimes^{d}$ in weight $d$ and with product $\sum_{s \in S(d, e)} \epsilon(\sigma) \sigma:\left(\otimes^{d}\right) \otimes$ $(\otimes e) \rightarrow \otimes^{d+e}$, where $S(d, e)$ is the set of $(d, e)$-shuffles.

Remark 4.7. In characteristic 2, [C2, Thm 3.2] asserts that the $\mathcal{P}_{\mathbb{k}}$-algebra $\mathbb{H}\left(\Lambda^{d}, \Gamma^{d}\right)$ is isomorphic to $\Lambda \otimes \Gamma^{(1)}$. The elementary computation of lemma 4.6 shows that this is false. (The problem in the proof of [C2, Thm 3.2] is that there is no reason why $v \mapsto \alpha_{s}^{(i)}(v)$ and $v \mapsto \beta_{s}^{(i)}(v)$ should be $\mathbb{k}$-linear).

We also have a convolution product (still denoted by $\star$ ) on the parameterized extension groups $\mathbb{E}(C, A)$ between a $\mathcal{P}_{\mathbb{k}}$-coalgebra and a $\mathcal{P}_{\mathbb{k}^{-}}$-graded algebra (compare [FFSS, p. 675]).

Definition 4.8. Let $C$ be a $\mathcal{P}_{\mathbb{k}}$-coalgebra and let $A$ be a $\mathcal{P}_{\mathbb{k}}$-algebra. We denote by $\mathbb{E}(C, A)$ the $\mathcal{P}_{\mathbb{k}}$-graded algebra defined as follows.

(i) The homogeneous part of degree $-i$ and weight $d$ of $\mathbb{E}(C, A)$ is the functor $\mathbb{E}^{i}\left(C_{0, d}, A_{0, d}\right)$.

(ii) The unit is the map $\mathbb{E}^{0}(\epsilon, \eta)=\mathbb{H}(\epsilon, \eta)$.

(iii) The convolution product $\star$ is defined as the composite:

$$
\begin{aligned}
\mathbb{E}^{i}\left(C_{0, d}, A_{0, d}\right) \otimes \mathbb{E}^{j}\left(C_{0, e}, A_{0, e}\right) \stackrel{\otimes}{\rightarrow} \mathbb{E}^{i+j}\left(C_{0, d} \otimes C_{0, e}, A_{0, d} \otimes A_{0, e}\right) \\
\stackrel{\mathbb{E}(\Delta, m)}{\longrightarrow} \mathbb{E}^{i+j}\left(C_{0, d+e}, A_{0, d+e}\right) .
\end{aligned}
$$

The Ext-algebras considered in FFSS and in C2 correspond to our strict polynomial graded algebras $\mathbb{E}\left(X^{(r)}, Y^{(s)}\right)$, for pairs $(X, Y)$ of classical exponential functors.

4.3. $(1, \epsilon)$-commutativity. The algebras $\mathbb{E}(C, A)$ are usually not graded commutative, even if $C$ and $A$ are commutative. Indeed, one easily checks the following lemma (cf. [FFSS, Lemma 1.11]).

Lemma 4.9. Let $X, Y$ be a pair of classical exponential functors and let $r, s$ be nonnegative integers (take $r=s=0$ if $\mathbb{k}$ is not a field of positive characteristic). Let $\epsilon(S)=\epsilon(\Gamma)=0$ and let $\epsilon(\Lambda)=1$. The following diagram commutes up to $a(-1)^{i j+\epsilon(X) k \ell+\epsilon(Y) m n}$ sign.

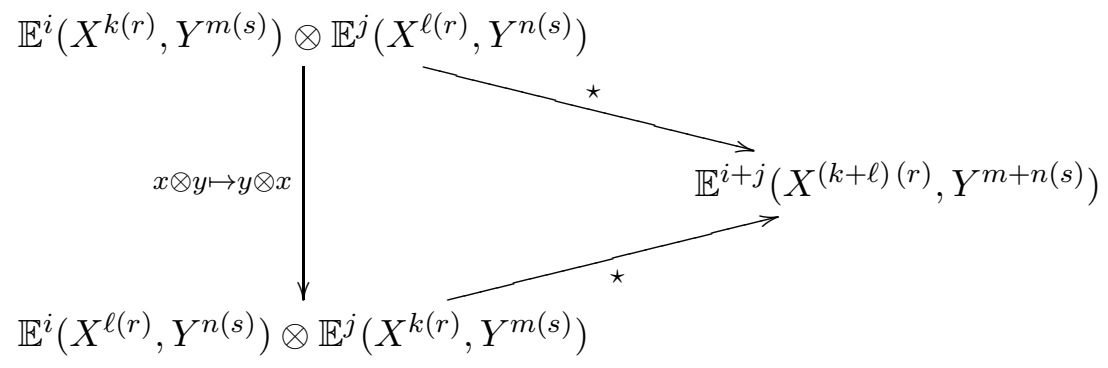

Proof. The $(-1)^{i j}$ sign is just the usual homological sign which comes from the commutativity of tensor products at the level of chain complexes $C \otimes D \simeq$ $D \otimes C$, see e.g. [W], proof of lemma 6.7.12]. The signs $(-1)^{\epsilon(X) k \ell}$ and 
$(-1)^{\epsilon(Y) m n}$ are the signs needed to have the following diagrams commute.

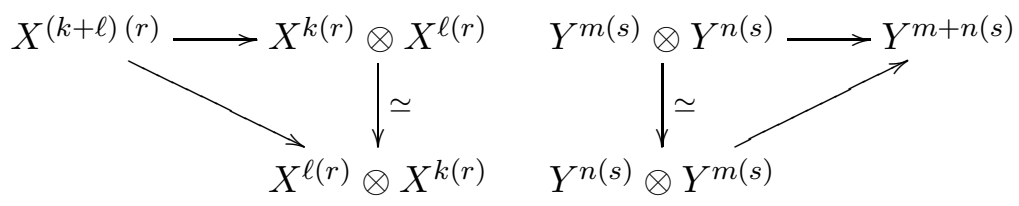

In order to formalize the graded commutativity defect of the convolution product $\star$, we make the following definition.

Definition 4.10. Let $A$ be a wdg-algebra, and let $\epsilon \in\{0,1\}$. We say that $A$ is $(1, \epsilon)$-commutative if for all homogeneous elements $x, y$ in $A$ we have:

$$
x \cdot y=(-1)^{|x||y|+\epsilon w(x) w(y)} y \cdot x .
$$

We say that a $\mathcal{P}_{\mathbb{k}}$-dg-algebra is $(1, \epsilon)$-commutative if for all $V \in \mathcal{V}_{\mathbb{k}}$, the wdg-algebra $A(V)$ is $(1, \epsilon)$-commutative.

Thus, the $(1,0)$-commutative $\mathcal{P}_{\mathbb{k}^{-}}$-dg-algebras are precisely the $\mathcal{P}_{\mathbb{k}^{-}}$-cdgalgebras. The $\mathcal{P}_{\mathbb{k}}$-graded algebras $S[i]$ and $\Gamma[i]$ are $(1,0)$-commutative for $i$ even, and $(1,1)$-commutative for $i$ odd, whereas $\Lambda[i]$ is $(1,1)$-commutative for $i$ even and $(1,0)$-commutative for $i$ odd. We reformulate lemma 4.9 in terms of $(1, \epsilon)$-commutativity.

Lemma 4.11. Let $X, Y$ be a pair of classical exponential functors and let $r, s$ be nonnegative integers (take $r=s=0$ if $\mathbb{k}$ is not a field of positive characteristic). Let $\epsilon(S)=\epsilon(\Gamma)=0$ and let $\epsilon(\Lambda)=1$. Let $\epsilon \in\{0,1\}$ such that $\epsilon=\epsilon(X)+\epsilon(Y)$ mod 2. Then $\mathbb{E}\left(X^{(r)}, Y^{(s)}\right)$ is $(1, \epsilon)$-commutative.

If $A$ and $B$ are wdg-algebras which are $(1,1)$-commutative, their tensor product $A \otimes B$ is not $(1,1)$-commutative. For this reason, we introduce a 'signed tensor product' $\otimes$.

Definition 4.12. Let $\epsilon \in\{0,1\}$. And let $A$ and $B$ be wdg-algebras. We define the wdg-algebra $A \otimes^{\epsilon} B$ in the following way.

(i) If $a \in A$ and $b \in B$ are homogeneous elements, the degree of $a \otimes b$ is defined by $|a \otimes b|=|a|+|b|$ and its weight by $w(a \otimes b)=w(a)+w(b)$.

(ii) The unit of $A \otimes^{\epsilon} B$ is the tensor product of the units of $A$ and $B$.

(iii) For all homogeneous elements $a \in A$ and $b \in B$, the differential of $A \otimes \otimes^{\epsilon} B$ is given by $d(a \otimes b)=d a \otimes b+(-1)^{|a|} a \otimes d b$.

(iv) For all homogeneous elements $a, a^{\prime} \in A$ and $b, b^{\prime} \in B$, the product of $A \otimes^{\epsilon} B$ is given by

$$
(a \otimes b) \cdot\left(a^{\prime} \otimes b^{\prime}\right)=(-1)^{\left|a^{\prime}\right||b|+\epsilon w\left(a^{\prime}\right) w(b)}\left(a a^{\prime}\right) \otimes\left(b b^{\prime}\right) .
$$

When $\epsilon=0$, the tensor product $\otimes^{\epsilon}$ is the usual tensor product of graded algebras. When $\epsilon=1$, the definition differs from the usual tensor product by the sign involved in the product. One defines a tensor product $\otimes^{\epsilon}$ on $\mathcal{P}_{\mathbb{k}}$-dg-algebras analogously.

Lemma 4.13. Let $A$ and $B$ be wdg-algebras. Then $A \otimes^{\epsilon} B$ is $(1, \epsilon)$ commutative if and only if $A$ and $B$ are $(1, \epsilon)$-commutative. 
Proof. If $A \otimes^{\epsilon} B$ is $(1, \epsilon)$-commutative, then $A$ (resp. $B$ ) identifies with the subalgebra of the elements of the form $a \otimes 1$ (resp. $1 \otimes b$ ), so it is also $(1, \epsilon)$-commutative. Conversely, let $A$ and $B$ be $(1, \epsilon)$-commutative. Let $a, a^{\prime} \in A$ and $b, b^{\prime} \in B$ be homogeneous elements. We denote $f(x, y)=$ $|x||y|+\epsilon w(x) w(y)$. We have

$$
\begin{aligned}
(a \otimes b) \cdot\left(a^{\prime} \otimes b^{\prime}\right) & =(-1)^{f\left(a^{\prime}, b\right)}\left(a a^{\prime}\right) \otimes\left(b b^{\prime}\right), \\
& =(-1)^{f\left(a^{\prime}, b\right)+f\left(a, a^{\prime}\right)+f\left(b, b^{\prime}\right)}\left(a^{\prime} a\right) \otimes\left(b^{\prime} b\right), \\
& =(-1)^{f\left(a^{\prime}, b\right)+f\left(a, a^{\prime}\right)+f\left(b, b^{\prime}\right)+f\left(a, b^{\prime}\right)}\left(a^{\prime} \otimes b^{\prime}\right) \cdot(a \otimes b) .
\end{aligned}
$$

The sign appearing on the last line equals $f\left(a \otimes b, a^{\prime} \otimes b^{\prime}\right)$, so $A \otimes^{\epsilon} B$ is $(1, \epsilon)$-commutative.

\section{The EXPOnEntial PROPERTy}

In this section, we recall the basics of exponential functors. Archetypes of exponential functors are symmetric, exterior and divided powers, this is why we call them 'classical exponential functors'. We prove that when $C$ and $A$ are exponential functors, the parameterized extension groups $\mathbb{E}(C, A)$ are exponential functors.

\subsection{Exponential functors.}

Definition 5.1. Let $A$ be a $\mathcal{P}_{\mathbb{k}}$-graded algebra. Then $A$ is called an exponential functor if (i) each summand $A_{i, d}$ of degree $i$ and weight $d$ takes values in $\mathcal{V}_{\mathbb{k}}$, and (ii) the following composite is an isomorphism of graded $\mathbb{k}$ modules (here $\iota_{V}$ and $\iota_{W}$ are the canonical inclusions of $V$ and $W$ in $V \oplus W$, and $m$ is the product in $A$ ).

$$
A(V) \otimes A(W) \stackrel{A\left(\iota_{V}\right) \otimes A\left(\iota_{W}\right)}{\longrightarrow} A(V \oplus W)^{\otimes 2} \stackrel{m}{\rightarrow} A(V \oplus W)
$$

Example 5.2. If $i$ is an integer, the $\mathcal{P}_{\mathbb{k}}$-graded algebras $S[i], \Lambda[i]$ and $\Gamma[i]$ from section 3.4 are exponential functors. Also, if $F$ is an additive functor (e.g. one of the functors $V \otimes, \operatorname{Hom}_{\mathbb{k}}(V,-)$ or $I^{(r)}$ from notation 2.2), and if $A$ is an exponential functor, then the composite $A(F)=A \circ F$ is an exponential functor.

We refer to the isomorphism $A(V \oplus W) \simeq A(V) \otimes A(W)$ as the exponential isomorphism. By definition, the multiplication of an exponential functor determines the exponential isomorphism. Conversely, if $A$ is an exponential functor, the multiplication of $A$ can be recovered from the exponential isomorphism, as the composite (where $\Sigma$ is the map $(x, y) \mapsto x+y$ ):

$$
A(V) \otimes A(V) \stackrel{\simeq}{\longrightarrow} A(V \oplus V) \stackrel{A(\Sigma)}{\longrightarrow} A(V) .
$$

Similarly, if $A$ is an exponential functor, the exponential isomorphism can also be used to define a comultiplication $\Delta$ on $A$, as the composite (where $\delta$ is the map $x \mapsto(x, x))$ :

$$
A(V) \stackrel{A(\delta)}{\longrightarrow} A(V \oplus V) \stackrel{\simeq}{\rightarrow} A(V) \otimes A(V) .
$$


And the exponential isomorphism can be recovered from the comultiplication $\Delta$ as the composite (where $\pi_{V}$ and $\pi_{W}$ denote the canonical projections)

$$
A(V \oplus W) \stackrel{\Delta}{\rightarrow} A(V \oplus W)^{\otimes 2} \stackrel{A\left(\pi_{V}\right) \otimes A\left(\pi_{W}\right)}{\longrightarrow} A(V) \otimes A(W) .
$$

So, if $A$ is an exponential functor, each one of the following data determines the two other ones: the multiplication, the exponential isomorphism, the comultiplication.

Example 5.3. For the classical exponential functors $X=S, \Lambda$ or $\Gamma$, the coproduct determined by the usual multiplication (i.e. the one defined by the canonical maps $X^{d} \otimes X^{e} \rightarrow X^{d+e}$ indicated in section 3.4) is the usual coproduct.

The following lemma is straightforward, we record it for further use.

Lemma 5.4. Let $A, B$ be exponential functors and let $f: A \rightarrow B$ be a morphism of graded functors. The following assertions are equivalent.

(i) $f$ is a morphism of $\mathcal{P}_{\mathbb{k}}$-graded algebras,

(ii) $f$ commutes with the exponential isomorphisms,

(iii) $f$ is a morphism of $\mathcal{P}_{\mathbb{k}}$-graded coalgebras.

5.2. The exponential property for extension groups. We now show that (under mild hypotheses), the parameterized extension groups $\mathbb{E}(C, A)$ between exponential functors are an exponential functor. We begin with a statement on the Hom-level.

Lemma 5.5. Let $E$ be an exponential functor, and let $F, G$ be strict polynomial functors with values in $\mathcal{V}_{\mathbb{k}}$. Let us denote $E_{i}$ by the summand of $E$ of degree $i: E_{i}=\bigoplus_{d} E_{i, d}$. Assume that for all $i, \mathbb{H}\left(E_{i}, F\right)$ and $\mathbb{H}\left(E_{i}, G\right)$ take finitely generated projective values. Then the composite is a graded isomorphism (where the morphism on the right is induced by the comultiplication of $E$, and the total degree is taken on the left hand side)

$\bigoplus_{i \geq 0} \mathbb{H}\left(E_{i}, F\right) \otimes \bigoplus_{j \geq 0} \mathbb{H}\left(E_{j}, G\right) \stackrel{\otimes}{\rightarrow} \bigoplus_{i, j \geq 0} \mathbb{H}\left(E_{i} \otimes E_{j}, F \otimes G\right) \rightarrow \bigoplus_{k \geq 0} \mathbb{H}\left(E_{k}, F \otimes G\right)$

Similarly, if the Hom-groups $\mathbb{H}\left(F, E_{i}\right)$ and $\mathbb{H}\left(G, E_{i}\right)$ are in $\mathcal{V}_{\mathbb{k}}$, the following composite (where the morphism on the right is induced by the multiplication of $E$, and the total degree is taken on the left hand side) is an isomorphism:

$$
\bigoplus_{i \geq 0} \mathbb{H}\left(F, E_{i}\right) \otimes \bigoplus_{j \geq 0} \mathbb{H}\left(G, E_{j}\right) \stackrel{\otimes}{\rightarrow} \bigoplus_{i, j \geq 0} \mathbb{H}\left(F \otimes G, E_{i} \otimes E_{j}\right) \rightarrow \bigoplus_{k \geq 0} \mathbb{H}\left(F \otimes G, E_{k}\right)
$$

Proof. The Kuhn dual of an exponential functor is an exponential functor. So by lemma 4.2, the first isomorphism of lemma 5.5] is equivalent to the second one via Kuhn duality. Thus we only prove the latter.

Fix $V \in \mathcal{V}_{\mathbb{k}}$ and denote by $A_{i, d}$ the functor $\left(E_{i, d}\right)_{V}$ and by $\left[F, A_{i, d}\right]$ the $\mathbb{k}$-module $\operatorname{Hom}_{\mathcal{P}_{\mathrm{k}}}\left(F, A_{i, d}\right)$. We have to prove that the following composite, called $\Phi$ in the sequel, is an isomorphism.

$$
\bigoplus_{i+j=k}\left[F, A_{i, d}\right] \otimes\left[G, A_{j, e}\right] \stackrel{\otimes}{\rightarrow} \bigoplus_{i+j=k}\left[F \otimes G, A_{i, d} \otimes A_{j, e}\right] \rightarrow\left[F \otimes G, A_{k, d+e}\right]
$$


Case $F=\Gamma^{d X}, G=\Gamma^{e Y}$. In this case, the source and the target of $\Phi$ are bifunctors with variables $X \in \Gamma^{d} \mathcal{V}_{\mathbb{k}}$ and $Y \in \Gamma^{e} \mathcal{V}_{\mathbb{k}}$, and $\Phi$ is a natural transformation of bifunctors. But $F \otimes G$ identifies as the direct summand of weight $d$ with respect to $X$ and weight $e$ with respect to $Y$ of the bifunctor $\Gamma^{\ell, X \oplus Y}$. So $\Phi$ is the homogeneous part of weight $d$ with respect to $X$ and $e$ with respect to $Y$ of the morphism (where the downwards map is induced by the multiplication of $A$ and by the diagonal $\left.\Gamma^{\ell X \oplus Y} \rightarrow \Gamma^{d X} \otimes \Gamma^{e Y}\right)$ :

$$
\begin{gathered}
\bigoplus_{\substack{i+j=k \\
d+e=\ell}}\left[\Gamma^{d X}, A_{i, d}\right] \otimes\left[\Gamma^{e Y}, A_{j, e}\right] \stackrel{\otimes}{\longrightarrow} \bigoplus_{\begin{array}{c}
i+j=k \\
d+e=\ell
\end{array}}\left[\Gamma^{d X} \otimes \Gamma^{e Y}, A_{i, d} \otimes A_{j, e}\right] \\
\qquad \\
{\left[\Gamma^{\ell X \oplus Y}, A_{k, \ell}\right]}
\end{gathered}
$$

The latter identifies through the Yoneda isomorphism with the composite

$$
\begin{gathered}
\bigoplus_{\substack{i+j=k \\
d+e=\ell}} A_{i, d}(X) \otimes A_{j, e}(Y) \stackrel{\otimes}{\longrightarrow} \bigoplus_{\begin{array}{c}
i+j=k \\
d+e=\ell
\end{array}} A_{i, d}(X \oplus Y) \otimes A_{j, e}(X \oplus Y) \\
\\
\\
A_{k, \ell}(X \oplus Y),
\end{gathered}
$$

where the downwards map is induced by the multiplication of $A(X \oplus Y)$. But $A=E_{V}$ is an exponential functor, so the latter composite is an isomorphism. Whence the result for $F=\Gamma^{d X}, G=\Gamma^{e Y}$.

General case. By additivity of (parameterized) Hom groups, we can restrict to the case of homogeneous functors $F \in \mathcal{P}_{d, \mathbb{k}}$ and $G \in \mathcal{P}_{e, \mathbb{k}}$. Now $\left[F, A_{i, d}\right]$ and $\left[G, A_{j, e}\right]$ are projective $\mathbb{k}$-modules, and so are $\left[\Gamma^{d, X}, A_{i, d}\right]$ and $\left[\Gamma^{e, Y}, A_{j, e}\right]$ (by the Yoneda lemma, since the homogeneous summands of $A$ have values in $\mathcal{V}_{\mathbb{k}}$ ). Hence the result for arbitrary $F, G$ follows from the result for $F=\Gamma^{d, X}$ and $G=\Gamma^{e, Y}$ by left exactness of Homs, when taking projective presentations of $F$ and $G$.

Remark 5.6. Alternatively, one can prove lemma 5.5 by using first the sumdiagonal adjunction as in the proof of [FFSS, Thm 1.7], and then identifying the isomorphism obtained, as in [T1, Lemma 5.13].

If $\mathbb{k}$ is a field, then the hypothesis that the Hom-groups $\mathbb{H}\left(E^{i}, F\right)$ and $\mathbb{H}\left(E^{i}, G\right)$ are finitely generated and projective over $\mathbb{k}$ is automatically satisfied. The next result follows from lemma 5.5 by taking projective resolutions and using the Künneth formula.

Lemma 5.7 (Compare [FFSS, Thm 1.7]). Let $\mathbb{k}$ be a field, let $E$ be an exponential functor, and let $F, G$ be strict polynomial functors with values in $\mathcal{V}_{\mathbb{k}}$. The following composite is an isomorphism.

$$
\bigoplus_{i+j=k} \mathbb{E}\left(F, E_{i}\right) \otimes \mathbb{E}\left(F, E_{j}\right) \stackrel{\otimes}{\rightarrow} \bigoplus_{i+j=k} \mathbb{E}\left(F \otimes G, E_{i} \otimes E_{j}\right) \rightarrow \mathbb{E}\left(F \otimes G, E_{k}\right)
$$

Proposition 5.8. Let $\mathbb{k}$ be a field, let $X, Y$ be exponential functors concentrated in degree 0 (i.e. $X_{i, d}=Y_{i, d}=0$ for $i>0$ ). The $\mathcal{P}_{\mathbb{k}}$-graded algebra $\mathbb{E}(X, Y)$ is a an exponential functor. 
Proof. First, since $\mathbb{k}$ is a field and the functors $X_{0, d}$ and $Y_{0, d}$ take finite dimensional values, the functors $\mathbb{E}^{i}\left(X_{0, d}, Y_{0, d}\right)$ take finite dimensional values. So it remains to check the exponential isomorphism. The following composite (where the morphism on the left hand side is induced by the canonical inclusions of $V, W$ in $V \oplus W$, and the morphism on the right hand side is given by the convolution product of $\mathbb{E}(X, Y))$ :

$$
\mathbb{E}(X, Y)(V) \otimes \mathbb{E}(X, Y)(W) \rightarrow \mathbb{E}(X, Y)(V \oplus W)^{\otimes 2} \rightarrow \mathbb{E}(X, Y)(V \oplus W)
$$

is an isomorphism. Indeed, it equals the composite

$$
\mathbb{E}\left(X, Y_{V}\right)(\mathbb{k}) \otimes \mathbb{E}\left(X, Y_{W}\right)(\mathbb{k}) \rightarrow \mathbb{E}\left(X, Y_{V} \otimes Y_{W}\right)(\mathbb{k}) \rightarrow \mathbb{E}\left(X, Y_{V \oplus W}\right)(\mathbb{k}),
$$

where the first map is the isomorphism of lemma 5.7 and the second one is induced by the isomorphism $Y_{V} \otimes Y_{W} \simeq Y_{V \oplus W}$.

Remark 5.9. We make no use of proposition 5.8 in this article. We have stated it only to justify that it is a priori not worthy to care about the coproduct on $\mathbb{E}(X, Y)$ as we claimed it in the introduction. Indeed, as observed in section 5.1, if we know $\mathbb{E}(X, Y)$ as a $\mathcal{P}_{\mathbb{k}}$-graded algebra, the coproduct is determined by the product (thus, on our computation in section 15, the obvious candidate for the coproduct is the good one!). Notice that such a reasoning does not work if one restricts to computing the unparameterized extension groups $\operatorname{Ext}_{\mathcal{P}_{\mathfrak{k}}}^{*}\left(X^{*}, Y^{*}\right)=\mathbb{E}(X, Y)(\mathbb{k})$, because the functoriality is needed to recover the coproduct.

\section{Part 2. Extension groups between $S, \Lambda, \Gamma$, and bar constructions}

In this part, $\mathbb{k}$ is a commutative ring. We compute the $\mathcal{P}_{\mathbb{k}}$-graded algebras $\mathbb{E}(S, \Lambda)$ and $\mathbb{E}(S, \Gamma)$ in terms of the homology of the (iterated) bar constructions of $\Gamma$. We first introduce regrading functors

$$
\mathcal{R}_{\alpha}:\left\{\mathcal{P}_{\mathbb{R}^{\mathbb{2}}} \text {-dg-alg }\right\} \rightarrow\left\{\mathcal{P}_{\mathbb{k}^{k}} \text {-dg-alg }\right\}
$$

These functors automatically take care of all the strange signs which arise in the computations. Then, we prove that $\mathbb{E}(S, \Lambda)$ and $\mathbb{E}(S, \Gamma)$ are equal to the homology of the $\mathcal{P}_{\mathbb{k}}$-dg-algebras ${ }^{t} \mathcal{R}_{2 i+1} \bar{B}(\Gamma[2 i])$ and $\mathcal{R}_{2 i+2} \bar{B}^{2}(\Gamma[2 i])$. This is done in theorem [7.5, which generalizes the main theorem of [A] The key point in the proof is the interchange property of proposition 7.2 , which is very specific to exponential functors.

\section{REgRADING FUnCTORS}

Definition 6.1. Let $\alpha$ be an integer and let $(A, \eta, m, \partial)$ be a strict polynomial differential graded algebra. The regraded algebra is the algebra $\left(\mathcal{R}_{\alpha} A, \mathcal{R}_{\alpha} \eta, \mathcal{R}_{\alpha} m, \mathcal{R}_{\alpha} \partial\right)$ defined by the following formulas.

(i) $\left(\mathcal{R}_{\alpha} A\right)_{i, d}=A_{i+\alpha d, d}$.

(ii) $\mathcal{R}_{\alpha} \eta=\eta$

(iii) $\mathcal{R}_{\alpha} m:\left(\mathcal{R}_{\alpha} A\right)_{i, d} \otimes\left(\mathcal{R}_{\alpha} A\right)_{j, e} \rightarrow\left(\mathcal{R}_{\alpha} A\right)_{i+j, d+e}$ equals $(-1)^{\alpha(i+d) e} m$.

(iv) $\mathcal{R}_{\alpha} \partial:\left(\mathcal{R}_{\alpha} A\right)_{i, d} \rightarrow\left(\mathcal{R}_{\alpha} A\right)_{i-1, d}$ equals $(-1)^{\alpha d} \partial$.

\footnotetext{
${ }^{3}$ The main theorem of $\mathrm{A}$ corresponds to the case of $\mathbb{E}(S, \Lambda)(\mathbb{k})$ (hence without functoriality), without the algebra structure.
} 
Let $V \in \mathcal{V}_{\mathbb{k}}$ be a finitely generated projective $\mathbb{k}$-module, and let $x, y$ be a pair of homogeneous elements of the weighted graded $\mathbb{k}$-module $A(V)$. We denote by $|x|$ the degree of $x$ in $A(V)$ and by $w(x)$ its weight. We denote by $s^{\alpha w(x)} x$ and $s^{\alpha w(y)} y$ the same elements, viewed as elements of the weighted graded $\mathbb{k}$-module $\mathcal{R}_{\alpha} A(V)$. Then the definitions of the product and of the differential take the more suggestive form (where the signs which appear are determined by the usual Koszul sign rule, see e.g. [LV, Chap 1]):

$$
\begin{aligned}
& s^{\alpha w(x)} x \cdot s^{\alpha w(y)} y:=(-1)^{\alpha|x| w(y)} s^{\alpha w(x \otimes y)} x \cdot y, \\
& \left(R_{\alpha} \partial\right)\left(s^{\alpha w(x)} x\right):=(-1)^{\alpha w(x)} s^{\alpha w(x)}(\partial x) .
\end{aligned}
$$

It is not hard to see from these formulas that the definition of $\mathcal{R}_{\alpha} A$ makes sense, i.e. that the product $\mathcal{R}_{\alpha} m$ is associative, and the differential $R_{\alpha} \partial$ is a derivation.

Definition 6.2. If $f: A \rightarrow B$ is a morphism of $\mathcal{P}_{\mathbb{k}}$-dg-algebras, we define $\mathcal{R}_{\alpha} f$ by $\left(\mathcal{R}_{\alpha} f\right)_{i, d}=f_{i+\alpha d, d}$. This yields a regrading functor:

$$
\mathcal{R}_{\alpha}:\left\{\mathcal{P}_{\mathbb{k}^{\mathbb{2}}} \text {-dg-alg }\right\} \rightarrow\left\{\mathcal{P}_{\mathbb{k}^{k}} \text {-dg-alg }\right\}
$$

Let us denote by $H$ the homology functor $H:\left\{\mathcal{P}_{\mathbb{k}^{-}}\right.$-dg-alg $\} \rightarrow\left\{\mathcal{P}_{\mathbb{k}}\right.$-g-alg $\}$, and recall from section 4.2 the convolution algebra functor $\mathbb{H}(C,-)$. It is easy to check that the regrading functor commutes with these two functors.

Lemma 6.3. Let $\alpha$ be an integer and let $C$ be a $\mathcal{P}_{\mathbb{k}}$-coalgebra. For all $\mathcal{P}_{\mathbb{k}}$ dg-algebra $A$, we have equalities of $\mathcal{P}_{\mathbb{k}}$-graded-algebras, and $\mathcal{P}_{\mathbb{k}}$-differential graded algebras:

$$
\mathcal{R}_{\alpha}(H A)=H\left(\mathcal{R}_{\alpha} A\right), \quad \mathcal{R}_{\alpha} \mathbb{H}(C, A)=\mathbb{H}\left(C, \mathcal{R}_{\alpha} A\right) .
$$

If $\alpha$ is even, one easily checks that $\mathcal{R}_{\alpha}$ is an isomorphism of categories, with inverse $\mathcal{R}_{-\alpha}$. This is not the case if $\alpha$ is odd. We first need to introduce a new definition.

Definition 6.4. Let $(A, \eta, m, \partial)$ be $\mathcal{P}_{\mathbb{k}}$-dg-algebra. The weight twisted algebra ${ }^{\mathrm{t}} A$ is the $\mathcal{P}_{\mathbb{k}^{k}}$-dg-algebra $\left(A, \eta,{ }^{\mathrm{t}} m, \partial\right)$ where ${ }^{t} m: A_{i, d} \otimes A_{j, e} \rightarrow A_{i+j, d+e}$ equals $(-1)^{d e} m$. If $f: A \rightarrow B$ is a morphism of algebras, we define ${ }^{\mathrm{t}} f=f$. This yields an involutive functor:

$$
{ }^{\mathrm{t}}:\left\{\mathcal{P}_{\mathbb{k}}-\mathrm{dg}-\mathrm{alg}\right\} \rightarrow\left\{\mathcal{P}_{\mathbb{k}^{\mathrm{k}}}-\mathrm{dg}-\mathrm{alg}\right\} .
$$

Lemma 6.5. Let $A, B$ be $\mathcal{P}_{\mathbb{k}^{-}}-d g$-algebras, and let $\epsilon \in\{0,1\}$.

(i) $A$ is $(1, \epsilon)$-commutative if and only if ${ }^{\mathrm{t}} A$ is.

(ii) There is an isomorphism of $\mathcal{P}_{\mathbb{k}}$-dg-algebras ${ }^{\mathrm{t}}\left(A \otimes{ }^{\epsilon} B\right) \simeq\left({ }^{\mathrm{t}} A\right) \otimes{ }^{\epsilon}\left({ }^{\mathrm{t}} B\right)$.

Proof. Let $V \in \mathcal{V}_{\mathbb{k}}$, and let $a, a^{\prime} \in A(V)$ and $b, b^{\prime} \in B(V)$ be homogeneous elements. Let us prove $(i)$. Since ${ }^{\mathrm{t}}$ is an involution, it suffices to prove the only if part. So we assume that $A$ is $(1, \epsilon)$-commutative. We denote by $a \cdot{ }^{\mathrm{t}} a^{\prime}$ the product in $A(V)$ and by $a \cdot a^{\prime}$ the product in $A(V)$. We have:

$$
\begin{aligned}
a \cdot{ }^{\mathrm{t}} a^{\prime}=(-1)^{w(a) w\left(a^{\prime}\right)} a \cdot a^{\prime} & =(-1)^{w(a) w\left(a^{\prime}\right)+|a|\left|a^{\prime}\right|+\epsilon w(a) w\left(a^{\prime}\right)} a^{\prime} \cdot a \\
& =(-1)^{|a|\left|a^{\prime}\right|+\epsilon w(a) w\left(a^{\prime}\right)} a^{\prime} \cdot{ }^{\mathrm{t}} a .
\end{aligned}
$$


Thus, ${ }^{\mathrm{t}} A$ is $(1, \epsilon)$-commutative. Now we turn to $(i i)$. Let $\psi(x, y)=$ $w(x) w(y)$. We define:

$$
\begin{aligned}
\Psi:{ }^{\mathrm{t}}\left(A \otimes \otimes^{\epsilon} B\right) & \rightarrow\left({ }^{\mathrm{t}} A\right) \otimes \otimes^{\epsilon}\left({ }^{\mathrm{t}} B\right) \\
a \otimes b & \mapsto(-1)^{\psi(a, b)} a \otimes b .
\end{aligned}
$$

It is straightforward to check that $\Psi$ preserves the degrees, the weights, the units and the differentials. We have to check that $\psi$ is multiplicative. We denote $f(x, y)=|x||y|+\epsilon w(x) w(y)$. $\operatorname{In}^{\mathrm{t}}\left(A \otimes \otimes^{\epsilon} B\right)$, the product is defined by:

$$
(a \otimes b) \cdot\left(a^{\prime} \otimes b^{\prime}\right)=(-1)^{\psi\left(a \otimes b, a^{\prime} \otimes b^{\prime}\right)+f\left(a^{\prime}, b\right)} a a^{\prime} \otimes b b^{\prime} .
$$

In $\left({ }^{\mathrm{t}} A\right) \otimes{ }^{\bar{\epsilon}}\left({ }^{\mathrm{t}} B\right)$, the product is defined by:

$$
(a \otimes b) \cdot\left(a^{\prime} \otimes b^{\prime}\right)=(-1)^{\psi(a, b)+\psi\left(a^{\prime}, b^{\prime}\right)+f\left(a^{\prime}, b\right)} a a^{\prime} \otimes b b^{\prime} .
$$

Then $\Psi\left((a \otimes b) \cdot\left(a \otimes b^{\prime}\right)\right)$ equals $\Psi(a \otimes b) \cdot \Psi\left(a \otimes b^{\prime}\right)$ up to a $(-1)^{n}$ sign, where $n$ equals the sum:

$\psi\left(a \otimes b, a^{\prime} \otimes b^{\prime}\right)+2 f\left(a^{\prime}, b\right)+\psi\left(a a^{\prime}, b b^{\prime}\right)+\psi(a, b)+\psi\left(a^{\prime}, b^{\prime}\right)+\psi\left(a, a^{\prime}\right)+\psi\left(b, b^{\prime}\right)$.

One readily checks that this sum is even. Hence $\psi$ is multiplicative.

Lemma 6.6. Let $\alpha$ be an integer. We have ${ }^{\mathrm{t}} \mathcal{R}_{\alpha}=\mathcal{R}_{\alpha}{ }^{\mathrm{t}}$. If $\alpha$ is even, we have $\mathcal{R}_{-\alpha} \mathcal{R}_{\alpha}=$ Id. If $\alpha$ is odd, we have $\mathcal{R}_{-\alpha} \mathcal{R}_{\alpha}={ }^{\mathrm{t}}$.

Proof. The proof that ${ }^{\mathrm{t}}$ and $\mathcal{R}_{\alpha}$ commute is straightforward. We concentrate on the identification of $\mathcal{R}_{-\alpha} \mathcal{R}_{\alpha}$. Let $A$ be a $\mathcal{P}_{\mathbb{k}^{k}}$-dg-algebra. It is clear that $\mathcal{R}_{-\alpha} \mathcal{R}_{\alpha} A=\left(A, \eta, \mathcal{R}_{-\alpha} \mathcal{R}_{\alpha} m, \partial\right)$, so we only have to identify the product of $\mathcal{R}_{-\alpha} \mathcal{R}_{\alpha} A$. Let $V \in \mathcal{V}_{\mathbb{k}}$, and let $x, y$ be homogeneous elements in $A(V)$. We define $\varpi(x)=\alpha w(x)$. Then we have:

$$
\begin{aligned}
s^{-\varpi(x)} s^{\varpi(x)} x \cdot s^{-\varpi(y)} s^{\varpi(y)} y & =(-1)^{-\varpi(y)(\varpi(x)+|x|)} s^{-\varpi(x \otimes y)}\left(s^{\varpi(x)} x \cdot s^{\varpi(y)} y\right), \\
& =(-1)^{-\varpi(y) \varpi(x)} s^{-\varpi(x \otimes y)} s^{\varpi(x \otimes y)} x \cdot y .
\end{aligned}
$$

If $\alpha$ is even, the sign in the latter equality equals one, so $\mathcal{R}_{-\alpha} \mathcal{R}_{\alpha} A=A$. If $\alpha$ is odd, the sign equals $(-1)^{w(x) w(y)}$ so $\mathcal{R}_{-\alpha} \mathcal{R}_{\alpha} A={ }^{\mathrm{t}} A$.

In particular, $\mathcal{R}_{\alpha}$ is an isomorphism of categories. The behavior of $\mathcal{R}_{\alpha}$ with respect to graded commutativity also depends on the parity of $\alpha$.

Lemma 6.7. Let $\alpha$ be an integer, let $\epsilon \in\{0,1\}$ and let $\bar{\epsilon} \in\{0,1\}$ such that $\bar{\epsilon}=\epsilon+1 \bmod 2$. Let $A$ be a $\mathcal{P}_{\mathbb{R}}$-dg-algebra.

- If $\alpha$ is even, then $\mathcal{R}_{\alpha} A$ is $(1, \epsilon)$-commutative if and only if $A$ is $(1, \epsilon)$-commutative.

- If $\alpha$ is odd, then $\mathcal{R}_{\alpha} A$ is $(1, \epsilon)$-commutative if and only if $A$ is $(1, \bar{\epsilon})$ commutative.

Proof. By lemma $6.5{ }^{\mathrm{t}}$ preserves $(1, \epsilon)$-commutativity. Thus, by lemma 6.6. it suffices to prove the 'only if' part of the statements. Let us assume that $A$ is $(1, \epsilon)$-commutative. Let $V \in \mathcal{V}_{\mathbb{k}}$. Let $x, y$ be homogeneous elements of $A(V)$. We define $\varpi(x)=\alpha w(x)$. Then we have:

$$
\begin{aligned}
s^{\varpi(y)} y \cdot s^{\varpi(x)} x & =(-1)^{|y| \varpi(x)} s^{\varpi(x \otimes y)} y \cdot x \\
& =(-1)^{|y| \varpi(x)+|x||y|+\epsilon w(x) w(y)} s^{\varpi(x \otimes y)} x \cdot y, \\
s^{\varpi(x)} x \cdot s^{\varpi(y)} y & =(-1)^{|x| \varpi(y)} s^{\varpi(x \otimes y)} x \cdot y .
\end{aligned}
$$


Thus $s^{\varpi(y)} y \cdot s^{\varpi(x)} x$ equals $s^{\varpi(x)} x \cdot s^{\varpi(y)} y$ up to a $(-1)^{n}$ sign, with

$$
\begin{aligned}
n & =|y| \varpi(x)+|x||y|+|x| \varpi(y)+\epsilon w(x) w(y) \\
& =(|x|+\varpi(x))(|y|+\varpi(y))+(\alpha+\epsilon) w(x) w(y) \quad \bmod 2 .
\end{aligned}
$$

But $|x|+\varpi(x)$ is the degree of $s^{\varpi(x)} x \in \mathcal{R}_{\alpha} A(V)$, thus the last equality shows that $\mathcal{R}_{\alpha} A$ is $(1, \epsilon+\alpha)$-commutative.

Lemma 6.8. Let $\alpha$ be an integer, let $\epsilon \in\{0,1\}$ and let $\bar{\epsilon} \in\{0,1\}$ such that $\bar{\epsilon}=\epsilon+1 \bmod 2$. If $\alpha$ is even, then for all $\mathcal{P}_{\mathbb{k}}-d g$-algebras $A, B$ there is an isomorphism of $\mathcal{P}_{\mathbb{k}}-d g$-algebras:

$$
R_{\alpha}\left(A \otimes^{\epsilon} B\right) \simeq \mathcal{R}_{\alpha}(A) \otimes^{\epsilon} \mathcal{R}_{\alpha}(B) .
$$

If $\alpha$ is odd, there is an isomorphism of $\mathcal{P}_{\mathbb{k}}-d g$-algebras:

$$
R_{\alpha}\left(A \otimes^{\epsilon} B\right) \simeq \mathcal{R}_{\alpha}(A) \otimes^{\bar{\epsilon}} \mathcal{R}_{\alpha}(B) .
$$

Proof. We do the proof for $\alpha$ odd (the case $\alpha$ even is elementary). We define $\varpi(x)=\alpha w(x)$. If $a, a^{\prime} \in A$ and $b, b^{\prime} \in B$ are homogeneous elements, we denote by $s^{\varpi(a \otimes b)} a \otimes b$ the element $a \otimes b$ viewed as an element of $R_{\alpha}\left(A \otimes^{\epsilon}\right.$ $B)$, and by $\left(s^{\varpi(a)} a\right) \otimes\left(s^{\varpi(b)} b\right)$ the element $a \otimes b$ viewed as an element of $\mathcal{R}_{\alpha}(A) \otimes \otimes^{\bar{\epsilon}} \mathcal{R}_{\alpha}(B)$. We also define $\phi(a, b)=|a| \varpi(b)$. It is straightforward to check that the map

$$
\begin{array}{cccc}
\Phi: & R_{\alpha}\left(A \otimes^{\epsilon} B\right) & \rightarrow & \mathcal{R}_{\alpha}(A) \otimes^{\bar{\epsilon}} \mathcal{R}_{\alpha}(B) \\
& s^{\varpi(a \otimes b)} a \otimes b & \mapsto & (-1)^{\phi(a, b)}\left(s^{\varpi(a)} a\right) \otimes\left(s^{\varpi(b)} b\right)
\end{array}
$$

preserves the degrees, the weights, the units and the differentials. We have to prove that $\Phi$ preserves the products. Let us write for short $f(x, y)=$ $|x||y|+\epsilon w(x) w(y)$ and $g(x, y)=(\varpi(x)+|x|)(\varpi(y)+|y|)+\bar{\epsilon} w(x) w(y)$. The multiplication in $R_{\alpha}\left(A \otimes^{\epsilon} B\right)$ is defined by:

$$
\begin{aligned}
\left(s^{\varpi(a \otimes b)} a \otimes b\right) \cdot & \left(s^{\varpi\left(a^{\prime} \otimes b^{\prime}\right)} a^{\prime} \otimes b^{\prime}\right) \\
& =(-1)^{\phi\left(a \otimes b, a^{\prime} \otimes b^{\prime}\right)} s^{\varpi\left(a \otimes b \otimes a^{\prime} \otimes b^{\prime}\right)}(a \otimes b) \cdot\left(a^{\prime} \otimes b^{\prime}\right), \\
& =(-1)^{\phi\left(a \otimes b, a^{\prime} \otimes b^{\prime}\right)+f\left(a^{\prime}, b\right)} s^{\varpi\left(a a^{\prime}\right)+\varpi\left(b b^{\prime}\right)}\left(a a^{\prime}\right) \otimes\left(b b^{\prime}\right) .
\end{aligned}
$$

The multiplication in $\mathcal{R}_{\alpha}(A) \otimes^{\bar{\epsilon}} \mathcal{R}_{\alpha}(B)$ is defined by:

$$
\begin{aligned}
\left(s^{\varpi(a)} a \otimes s^{\varpi(b)} b\right) \cdot & \left(s^{\varpi\left(a^{\prime}\right)} a^{\prime} \otimes s^{\varpi\left(b^{\prime}\right)} b^{\prime}\right) \\
& =(-1)^{g\left(b, a^{\prime}\right)}\left(s^{\varpi(a)} a \cdot s^{\varpi\left(a^{\prime}\right)} a^{\prime}\right) \otimes\left(s^{\varpi(b)} b \cdot s^{\varpi\left(b^{\prime}\right)} b^{\prime}\right), \\
& =(-1)^{g\left(b, a^{\prime}\right)+\phi\left(a, a^{\prime}\right)+\phi\left(b, b^{\prime}\right)}\left(s^{\varpi\left(a a^{\prime}\right)} a a^{\prime}\right) \otimes\left(s^{\varpi\left(b b^{\prime}\right)} b b^{\prime}\right) .
\end{aligned}
$$

Thus, we obtain:

$\Phi\left(s^{\varpi(a \otimes b)} a \otimes b \cdot s^{\varpi\left(a^{\prime} \otimes b^{\prime}\right)} a^{\prime} \otimes b^{\prime}\right)=(-1)^{n} \Phi\left(s^{\varpi(a \otimes b)} a \otimes b\right) \cdot \Phi\left(s^{\varpi\left(a^{\prime} \otimes b^{\prime}\right)} a^{\prime} \otimes b^{\prime}\right)$,

where the integer $n$ equals the following sum:

$g\left(b, a^{\prime}\right)+\phi\left(a, a^{\prime}\right)+\phi\left(b, b^{\prime}\right)+\phi\left(a \otimes b, a^{\prime} \otimes b^{\prime}\right)+f\left(a^{\prime}, b\right)+\phi(a, b)+\phi\left(a^{\prime}, b^{\prime}\right)+\phi\left(a a^{\prime}, b b^{\prime}\right)$.

One readily checks that this sum is even. So $\Phi$ preserves products. 
We finish this section by an explicit computation involving $\mathcal{R}_{\alpha}$. Recall that $X[i]$ denotes the $\mathcal{P}_{\mathbb{k}^{-}}$-graded algebra with $X^{d}$ in degree $d i$ and weight $d$, equipped with multiplication given by the canonical maps $X^{d} \otimes X^{e} \rightarrow X^{d+e}$. We also denote by $X[i]^{(r)}$ the $\mathcal{P}_{\mathbb{k}^{k}}$-graded algebra $X[i]$ precomposed by the Frobenius twist (so it has $X^{d(r)}$ in degree $d i$ and weight $p d$ ).

Lemma 6.9. Let $X$ be $S, \Lambda$ or $\Gamma$. Assume that $\alpha$ is even or that $i$ is even. Then we have the following equalities of $\mathcal{P}_{\mathbb{k}}$-graded algebras.

$$
R_{\alpha}\left(X[i]^{(r)}\right)=X\left[i-\alpha p^{r}\right] .
$$

Assume that both $\alpha$ and $i$ are odd. Then we have the following equalities of $\mathcal{P}_{\mathbb{k}}$-graded algebras.

$$
R_{\alpha}\left(X[i]^{(r)}\right)={ }^{\mathrm{t}}\left(X\left[i-\alpha p^{r}\right]^{(r)}\right) .
$$

Proof. The equalities of lemma 6.9 are straightforward as equalities of graded strict polynomial functors. Let us identify the multiplication on $R_{\alpha}\left(X[i]^{(r)}\right)$. Let $V \in \mathcal{V}_{\mathbb{k}}$ and let $x, y$ be homogeneous elements in $X[i]^{(r)}(V)$. Then:

$$
s^{\alpha w(x)} x \cdot s^{\alpha w(y)} y=(-1)^{\alpha|x| w(y)} s^{\alpha w(x \otimes y)} x \cdot y .
$$

If $\alpha$ is even the sign equals 1 . If alpha is odd, and $i$ is even, all elements of $X[i](V)$ are in even degree, so the sign also equals 1 . If $\alpha$ and $i$ are odd, then $\alpha|x|=w(x) \bmod 2$, so the sign equals $(-1)^{w(x) w(y)}$. Whence the result.

\section{BAR CONSTRUCTIONS AND EXTENSION GROUPS}

7.1. Recollections of bar constructions. Let $A$ be a differential graded augmented $\mathbb{k}$-algebra (dga-k-algebra). We denote by $\epsilon$ its augmentation and by $A^{\prime}=\operatorname{ker} \epsilon$ the augmentation ideal of $A$. The degree of an homogeneous element $a \in A$ is denoted by $|a|$. The (reduced, normalized) bar construction over $A$ is the differential graded coaugmented $\mathbb{k}$-coalgebra $\bar{B} A$ defined as follows see e.g. [ML, X.10] or [LV, Chap. 2].

- $\bar{B} A$ equals $\bigoplus_{n \geq 0} A^{\prime \otimes n}$ as a $\mathbb{k}$-module.

- A scalar $\lambda$ of $\mathbb{k}=A^{\otimes \otimes 0}$ is denoted by $\lambda[]$ and has degree 0 . For $n \geq 1$, let $a_{i}$ be homogeneous elements of $A, 1 \leq i \leq n$. The element $a_{1} \otimes \cdots \otimes a_{n} \in A^{\prime \otimes n}$ is denoted by $\left[a_{1}|\ldots| a_{n}\right]$ and has degree $n+\sum\left|a_{i}\right|$.

- The counit is the map $\bar{B} A \rightarrow A^{\prime \otimes 0}=\mathbb{k}$, and the coaugmentation is the map $\mathbb{k}=A^{\prime \otimes 0} \hookrightarrow \bar{B} A$.

- The differential $\partial: \bar{B} A_{k} \rightarrow \bar{B} A_{k-1}$ sends an element $\left[a_{1}|\ldots| a_{n}\right]$ to the sum:

$$
\sum_{i=1}^{n-1}(-1)^{e_{i}}\left[a_{1}|\ldots| a_{i} a_{i+1}|\ldots| a_{n}\right]-\sum_{i=1}^{n}(-1)^{e_{i-1}}\left[a_{1}|\ldots| \partial a_{i}|\ldots| a_{n}\right],
$$

where $e_{0}=0$ and for $i \geq 1, e_{i}$ equals $i+\sum_{j \leq i}\left|a_{i}\right|$.

- The coproduct $\Delta: \bar{B} A \rightarrow \bar{B} A \otimes \bar{B} A$ sends an element $\left[a_{1}|\ldots| a_{n}\right]$ to the sum

$$
\sum_{i=0}^{n}\left[a_{1}|\ldots| a_{i}\right] \otimes\left[a_{i+1}|\ldots| a_{n}\right] .
$$


When $A$ is graded commutative, we can define a 'shuffle product' on $\bar{B} A$, which makes $\bar{B} A$ into a cdga-k-algebra. So we can iterate bar constructions, and we denote by $\bar{B}^{n} A$ the $n$-th iterated bar construction of $A$. To be more specific, if $a_{i}$ are homogeneous elements of $A$, the shuffle product $\left[a_{1}|\ldots| a_{p}\right] *$ $\left[a_{p+1}|\ldots| a_{p+q}\right]$ equals

$$
\sum \epsilon(\sigma)\left[a_{\sigma^{-1}(1)}|\ldots| a_{\sigma^{-1}(p+q)}\right]
$$

where the sum is taken over all $(p, q)$-shuffles $\sigma$, and $\epsilon(\sigma)$ is the Koszul sign such that $x_{1} \wedge \cdots \wedge x_{n}=\epsilon(\sigma) x_{\sigma(1)} \wedge \cdots \wedge x_{\sigma(n)}$ in the exterior algebra $\Lambda\left(x_{1}, \ldots, x_{n}\right)$ over generators $x_{i}$ with degree $\left|a_{i}\right|+1$.

We have presented the (reduced, normalized) bar construction of a dga$\mathbb{k}$-algebra, but the formulas above also make sense for the other categories of algebras described in section 3 (if the algebras have weights, the weights in $\bar{B} A$ are defined by $\left.w\left(\left[a_{1}|\ldots| a_{n}\right]\right)=\sum w\left(a_{i}\right)\right)$. In particular, for $\mathcal{P}_{\mathbb{k}^{-}}$-cdgaalgebras, iterated bar constructions yield functors $(n \geq 0)$ :

$$
\left\{\mathcal{P}_{\mathbb{k}^{\mathrm{k}}} \text {-cdga-alg }\right\} \stackrel{\bar{B}^{n}}{\longrightarrow}\left\{\mathcal{P}_{\mathbb{k}^{\mathrm{k}}} \text {-cdga-alg }\right\} .
$$

7.2. Bar constructions of symmetric and exterior algebras. Let us now concentrate on the concrete example of bar constructions of symmetric and exterior algebras. Recall that for all integer $i, S[i]$ (resp. $\Lambda[i]$ ) denotes the $\mathcal{P}_{\mathbb{k}}$-graded algebra, with $S^{d}$ (resp. $\Lambda^{d}$ ) in degree $d i$ and weight $d$. We can write down explicitly $\bar{B}(S[i])$. For $d \geq 0$, the homogeneous part of weight $d$ of $\bar{B}(S[i])$ is the following complex of strict polynomial functors, which we denote by $I_{\bullet, d}$ :

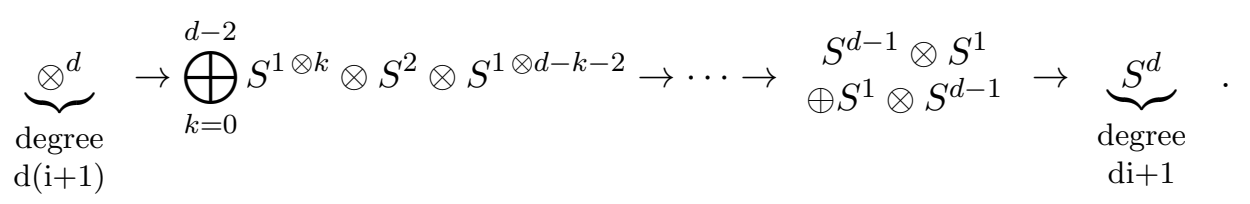

Similarly, one gets the homogeneous part of weight $d$ of $\bar{B}(\Lambda[i])$ by replacing symmetric powers by exterior ones in the complex above.

Assume that $S[i]$, resp. $\Lambda[i]$, are graded commutative (i.e. assume that $i$ is even, resp. odd, or that $\mathbb{k}$ has characteristic 2$)$. Then the canonical inclusions $\Lambda^{d} \hookrightarrow \otimes^{d}$, resp. $\Gamma^{d} \hookrightarrow \otimes^{d}$, define morphisms of $\mathcal{P}_{\mathbb{k}}$-cdga-algebras:

$$
\Lambda[i+1] \hookrightarrow \bar{B}(S[i]) \quad \text { and } \quad \Gamma[i+1] \hookrightarrow \bar{B}(\Lambda[i])
$$

These morphisms are quasi-isomorphisms, see e.g. [LV, Chap 3, ex 3.2.5]. In particular, the complex $I_{\bullet, d}$ drawn above yields a coresolution of $\Lambda^{d}$ by symmetric powers.

Let us recall from [ML, X Th. 11.2] that bar constructions preserve quasi isomorphisms of algebras. Hence, the composite

$$
\Gamma[i+2] \hookrightarrow \bar{B}(\Lambda[i+1]) \hookrightarrow \bar{B}^{2}(S[i])
$$

is a quasi isomorphism. So the homogeneous part of weight $d$ of $\bar{B}^{2}(S[i])$ yields a complex $J_{\bullet, d}$ of symmetric powers, whose homology equals $\Gamma^{d}$ in 
degree $d(i+2)$ (and zero in other degrees). The complex $J_{\bullet, d}$ has the form:

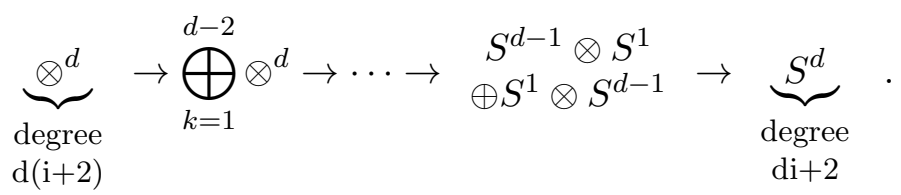

By lemma 2.3, we can use the complexes $I_{\bullet, d}$ and $J_{\bullet, d}$ to compute $\mathbb{E}\left(F, S^{d}\right)$ and $\mathbb{E}\left(F, \Lambda^{d}\right)$ when $F$ takes values in the category $\mathcal{V}_{\mathbb{k}}$ of finitely generated projective $\mathbb{k}$-modules.

Proposition 7.1. Let $\mathbb{k}$ be a commutative ring, let $C$ be a $\mathcal{P}_{\mathbb{k}}$-coalgebra whose homogeneous components $C_{d, i}$ take values in $\mathcal{V}_{\mathbb{k}}$, and let $i$ be a nonnegative integer. The $\mathcal{P}_{\mathbb{k}}$-graded algebras $\mathbb{E}(C, \Lambda)$ and $\mathbb{E}(C, \Gamma)$ are respectively isomorphic to the homology of the convolution $\mathcal{P}_{\mathbb{k}}$-dg-algebras ${ }^{\mathrm{t}} \mathcal{R}_{2 i+1} \mathbb{H}(C, \bar{B}(S[2 i]))$ and $\mathcal{R}_{2 i+2} \mathbb{H}\left(C, \bar{B}^{2}(S[2 i])\right)$.

Proof. We prove the case of $\mathbb{E}(C, \Lambda)$, the other case is similar. Let $K_{d}$ be a coresolution of $\Lambda^{d}$ by symmetric powers. Then $\mathbb{E}\left(C_{0, d}, \Lambda^{d}\right)$ is the homology of the complex $\mathbb{H}\left(C_{0, d}, K_{d}\right)$, and the convolution product is given on the cochain level by the composite:

$\mathbb{H}\left(C_{0, d}, K_{d}\right) \otimes \mathbb{H}\left(C_{0, e}, K_{e}\right) \stackrel{\otimes}{\rightarrow} \mathbb{H}\left(C_{0, d} \otimes C_{0, e}, K_{d} \otimes K_{e}\right) \stackrel{\mathbb{H}\left(\Delta_{d, e}, \widetilde{m}\right)}{\longrightarrow} \mathbb{H}\left(C_{0, d+e}, K_{d+e}\right)$, where $\Delta_{d, e}$ is the comultiplication $C_{0, d+e} \rightarrow C_{0, d} \otimes C_{0, e}$ and $\widetilde{m}: K_{d} \otimes K_{e} \rightarrow$ $K_{d+e}$ is a lifting of the multiplication $m: \Lambda^{d} \otimes \Lambda^{e} \rightarrow \Lambda^{d+e}$.

Consider the $\mathcal{P}_{\mathbb{k}^{k}}$-dg-algebra ${ }^{\mathrm{t}} \mathcal{R}_{2 i+1}(\bar{B} S[2 i])$. It is quasi isomorphic to ${ }^{\mathrm{t}} \mathcal{R}_{2 i+1}(\Lambda[2 i+1])=\Lambda$. So we may take for $K_{d}$ the homogeneous part of weight $d$ of this $\mathcal{P}_{\mathbb{k}^{k}}$-dg-algebra (this is $I_{\bullet}, d$, shifted), and for $\widetilde{m}$ the multiplication of ${ }^{\mathrm{t}} \mathcal{R}_{2 i+1}(\bar{B} S[2 i])$. With this choice, the composite above is the convolution product of $\mathbb{H}\left(C,{ }^{\mathrm{t}} \mathcal{R}_{2 i+1}(\bar{B} S[2 i])\right)$. Thus, $\mathbb{E}(C, \Lambda)$ equals the homology of $\mathbb{H}\left(C,{ }^{\mathrm{t}} \mathcal{R}_{2 i+1}(\bar{B} S[2 i])\right)$. By lemma 6.3, the algebras ${ }^{\mathrm{t}} \mathcal{R}_{2 i+1} \mathbb{H}(C, \bar{B} S[2 i])$ and $\mathbb{H}\left(C,{ }^{\mathrm{t}} \mathcal{R}_{2 i+1}(\bar{B} S[2 i])\right)$ are equal, whence the result.

7.3. The interchange property. This subsection is the core of our computation. Recall from section 4.2 that if $C$ is a coaugmented commutative $\mathcal{P}_{\mathbb{k}}$-coalgebra, we have a convolution algebra functor:

$$
\mathbb{H}(C,-):\left\{\mathcal{P}_{\mathbb{k}^{-}} \text {-cdga-alg }\right\} \rightarrow\left\{\mathcal{P}_{\mathbb{k}} \text {-cdga-alg }\right\} .
$$

The following key result asserts that when $C$ is exponential, the functor $\mathbb{H}(C,-)$ commutes with the bar construction functor $\bar{B}:\left\{\mathcal{P}_{\mathbb{k}^{-}}\right.$-cdga-alg $\} \rightarrow$ $\left\{\mathcal{P}_{\mathbb{k}}\right.$-cdga-alg $\}$.

Proposition 7.2 (Interchange property). Let $\mathbb{k}$ be a commutative ring, let $E=\left\{E_{0, d}\right\}_{d \geq 0}$ be a commutative exponential functor concentrated in degree zero, and let $A=\left\{A_{i, d}\right\}_{i, d \geq 0}$ be a $\mathcal{P}_{\mathbb{l k}^{\mathrm{k}}}$-cdga-algebra. Assume that $\mathbb{H}(E, A)$ takes $\mathbb{k}$-projective values. Then there is an isomorphism of $\mathcal{P}_{\mathbb{k}}$-cdga-algebras, natural with respect to $A$ :

$$
\mathbb{H}(E, \bar{B} A) \simeq \bar{B} \mathbb{H}(E, A) .
$$

Remark 7.3. We encourage the reader to try to prove a statement similar to proposition 7.2 in the framework of Schur algebras to understand the 
subtle difference between strict polynomial functors and modules over Schur algebras.

Remark 7.4. If $\mathbb{k}$ is a Dedekind domain (e.g $\mathbb{k}$ is a field or $\mathbb{Z}$ ), and $A$ has $\mathbb{k}$-projective values, the technical assumption that $\mathbb{H}(E, A)$ has $\mathbb{k}$-projective values is always satisfied.

Proof of proposition 7.2. Let us write for short $[X]$ instead of $\mathbb{H}(E, X)$. Lemma $\left[5.5\right.$ yields an isomorphism $\theta_{n}:[A]^{\otimes n} \rightarrow\left[A^{\otimes n}\right]$. Taking the direct sum over all $n \geq 0$, we get an isomorphism of graded strict polynomial functors: $\theta: \bar{B}[A] \stackrel{\cong}{\rightrightarrows}[\bar{B} A]$, natural with respect to $A$. We have to check that $\theta$ is an isomorphism of $\mathcal{P}_{\mathbb{k}}$-cdga-algebras. It is obvious that $\theta$ preserves augmentations.

By definition, the product $\star$ in the convolution algebra $[A]$ is the composite $[m] \circ \theta_{2}$, where $m$ is the multiplication of $A$. Thus we have a commutative diagram for all $k \leq n$ :

$$
\begin{aligned}
& \begin{array}{c}
{[A]^{\otimes n} \stackrel{\simeq}{\theta_{n}}\left[A^{\otimes n}\right]} \\
{[A]^{\otimes k} \otimes * \otimes[A]^{\otimes n-k-2} \mid}
\end{array} \\
& {[A]^{\otimes k} \otimes[A] \otimes[A]^{\otimes n-k-2} \underset{\theta_{n-1}}{\simeq}\left[A^{\otimes n-1}\right] .}
\end{aligned}
$$

By definition of the differentials of $[\bar{B} A]$ and $\bar{B}[A]$, this commutative diagram implies that $\theta$ commutes with the differentials.

Let $\sigma \in \mathfrak{S}_{n}$. Since $E$ is commutative, we have a commutative diagram:

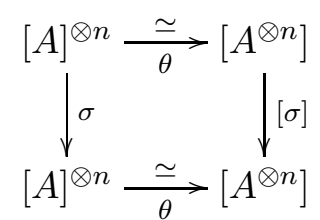

where $\sigma:[A]^{\otimes n} \rightarrow[A]^{\otimes n}$ and $[\sigma]:\left[A^{\otimes n}\right] \rightarrow\left[A^{\otimes n}\right]$ are the maps induced by permuting the factors of the tensor products. By definition of the products of $\bar{B}[A]$ and $[\bar{B} A]$, this commutative diagram implies that $\theta$ is an isomorphism of algebras. Whence the result.

Theorem 7.5. Let $\mathbb{k}$ be a commutative ring, and let $i$ an integer. The $\mathcal{P}_{\mathbb{k}}$-graded algebras $\mathbb{E}(S, \Lambda)$ and $\mathbb{E}(S, \Gamma)$ are respectively computed by the homology of the $\mathcal{P}_{\mathbb{k}}$-dg-algebras ${ }^{\mathrm{t}} \mathcal{R}_{2 i+1} \bar{B}(\Gamma[2 i])$ and $\mathcal{R}_{2 i+2} \bar{B}^{2}(\Gamma[2 i])$.

Proof. We first observe that $\mathbb{H}(S, S[2 i])=\Gamma[2 i]$, hence has $\mathbb{k}$-projective values. Thus, proposition 7.2 and lemma 4.6 yield an isomorphism:

$$
\mathbb{H}(S, \bar{B}(S[2 i])) \simeq \bar{B} \mathbb{H}(S, S[2 i]) \simeq \bar{B}(\Gamma[2 i]) .
$$

But $\Gamma[2 i]$, hence also $\bar{B}(\Gamma[2 i])$ has $\mathbb{k}$-projective values. Thus, by the isomorphism above, $\mathbb{H}(S, \bar{B}(S[2 i]))$ has $\mathbb{k}$-projective values. Applying proposition 7.2 again, we get an isomorphism:

$$
\mathbb{H}\left(S, \bar{B}^{2}(S[2 i])\right) \simeq \bar{B} \mathbb{H}(S, \bar{B}(S[2 i])) \simeq \bar{B}^{2}(\Gamma[2 i]) .
$$

Now the result follows from proposition 7.1 . 
Remark 7.6. In proposition 7.2, it is essential that $E$ is commutative. If this is not the case, then $\mathbb{H}(E, A)$ is not graded commutative, so $\bar{B} \mathbb{H}(E, A)$ does not bear a multiplication. However, it is easy to check (same proof) that if we drop the commutativity of $E$, we still have an isomorphism of complexes of strict polynomial functors:

$$
\mathbb{H}(E, \bar{B} A) \simeq \bar{B} \mathbb{H}(E, A) .
$$

Applying this to $E=A=\Lambda$, we get an isomorphism of complexes $\mathbb{H}(\Lambda, \bar{B} S) \simeq \bar{B} \mathbb{H}(\Lambda, S)$. But $\mathbb{H}(\Lambda, S) \simeq \Lambda$, so the homology of $\mathbb{H}(\Lambda, \bar{B} S)$ equals $\Gamma[1]$ (as a graded strict polynomial functor). Since the homology of $\mathcal{R}_{1}(\mathbb{H}(\Lambda, \bar{B} S)$ ) computes $\mathbb{E}(\Lambda, \Lambda)$ (same proof as proposition 7.1, without taking the algebra structure into account), we finally obtain (cf. lemma 4.6):

$$
\mathbb{E}(\Lambda, \Lambda)=\mathbb{H}(\Lambda, \Lambda) \simeq \Gamma .
$$

This provides a proof that there are no extension groups between $\Lambda$ and $\Lambda$, as asserted in the introduction.

\section{Part 3. Homology of Eilenberg-Mac Lane spaces}

In theorem 7.5 in part 2, we proved that $\mathbb{E}(S, \Lambda)$ and $\mathbb{E}(S, \Gamma)$ are equal (up to regrading) to the homology of iterated bar constructions of $\Gamma[2]$. In part 3, we tackle the following problem.

Problem 1. Compute the homology of the differential graded $\mathcal{P}_{\mathbb{k}}$-algebras $\bar{B}^{n}(\Gamma[2])$.

\section{Bar constructions and Eilenberg-Mac Lane spaces}

Akin observed [A] that over a field $\mathbb{k}$, the homology of $\bar{B} \Gamma(\mathbb{k}[2])$ can be interpreted as the dual of the rational cohomology of the additive group, computed in [CPSvdK]. However, this approach solves problem only for $n=1$ and when the ground ring $\mathbb{k}$ is a field. To solve problem 1 in general, we rather interpret the homology of $\bar{B}^{n}(\Gamma[2])$ as the singular homology of some Eilenberg Mac Lane (EML) spaces. Indeed, for an abelian group $\pi$, the singular homology with coefficients in $\mathbb{k}$ of the EML space $K(\pi, n)$ is isomorphic [EML] to the homology of $\bar{B}^{n}(\mathbb{k} \pi)$, where $\mathbb{k} \pi$ denotes the group algebra of $\pi$. When $\pi$ is free abelian, we can choose a $\mathbb{k}$-linear section of the canonical map $\mathbb{k} \pi \rightarrow \pi \otimes_{\mathbb{Z}} \mathbb{k}$. By the universal property of the symmetric algebra, this section induces a morphism of $\mathbb{k}$-algebras $\psi: S\left(\pi \otimes_{\mathbb{Z}} \mathbb{k}\right) \rightarrow \mathbb{k} \pi$.

Proposition 8.1. Let $\mathbb{k}$ be a commutative ring, let $\pi$ be a free abelian group, and let $\psi: S\left(\pi \otimes_{\mathbb{Z}} \mathbb{k}\right) \rightarrow \mathbb{k} \pi$ be a morphism of $\mathbb{k}$-algebras build from a section of the canonical map $\mathbb{k} \pi \rightarrow \pi \otimes_{\mathbb{Z}} \mathbb{k}$. Then for all positive integer $n$, the map

$$
\bar{B}^{n}(\psi): \bar{B}^{n}\left(S\left(\pi \otimes_{\mathbb{Z}} \mathbb{k}\right)\right) \rightarrow \bar{B}^{n}(\mathbb{k} \pi)
$$

is a quasi-isomorphism. It is not natural with respect to $\pi$, however the map $H\left(\bar{B}^{n}(\psi)\right)$ induced on homology is natural with respect to $\pi$.

Proof. Let us first prove that $\bar{B}(\psi)$ is a quasi-isomorphism. Let $s$ denote the section of the canonical map $q: \mathbb{k} \pi \rightarrow \pi \otimes_{\mathbb{Z}} \mathbb{k}$. Since all the elements of $\bar{B}(\mathbb{k} \pi)_{1}=\mathbb{k} \pi$ are cycles and the differential graded algebra $\bar{B}(\mathbb{k} \pi)$ is graded 
commutative, products yield a morphism of differential graded $\mathbb{k}$-algebras (take the trivial differential on the left hand side)

$$
\Lambda(s): \Lambda\left(\pi \otimes_{\mathbb{Z}} \mathbb{k}[1]\right) \rightarrow \bar{B}(\mathbb{k} \pi) .
$$

It is well known that $\Lambda(s)$ is a quasi-isomorphism, see e.g. Br, V.6 Th 6.4(ii)]. Now $\bar{B}(\psi)$ is a morphism of algebras, so $\Lambda(s)$ factors as:

$$
\Lambda\left(\pi \otimes_{\mathbb{Z}} \mathbb{k}[1]\right) \hookrightarrow \bar{B}\left(S\left(\pi \otimes_{\mathbb{Z}} \mathbb{k}\right)\right) \stackrel{\bar{B}(\psi)}{\longrightarrow} \bar{B}(\mathbb{k} \pi)
$$

As recalled in section 7.2 the first map is a quasi-isomorphism, and since $\Lambda(s)$ is a quasi-isomorphism, so is $\bar{B}(\psi)$. Bar constructions preserve quasiisomorphisms, so for all $n \geq 1, \bar{B}^{n}(\psi)$ is a quasi-isomorphism.

It remains to show that the map induced on homology by $\bar{B}^{n}(\psi)$ is natural with respect to $\pi$. We have a commutative diagram of differential graded $\mathbb{k}$-algebras:

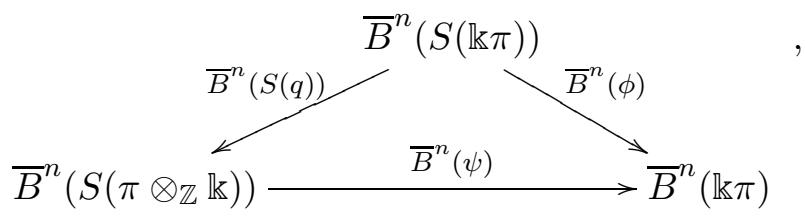

where $\phi: S(\mathbb{k} \pi) \rightarrow \mathbb{k} \pi$ is the morphism of $\mathbb{k}$-algebras induced by the universal property of the symmetric algebra and the identity map of $\mathbb{k} \pi$. The map $\bar{B}^{n}(S(q))$ is surjective in homology, indeed $\bar{B}^{n}(S(s))$ provides a section of $\bar{B}^{n}(S(q))$. Since $\bar{B}^{n}(\psi)$ is a quasi-isomorphism, $\bar{B}^{n}(\phi)$ is also surjective in homology. So for all $\pi$ we have a commutative diagram:

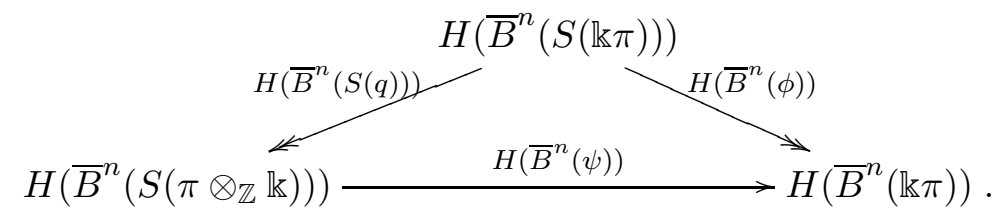

By their definition, the two maps with source $H\left(\bar{B}^{n}(S(\mathbb{k} \pi))\right)$ are natural with respect to $\pi$. Since they are surjective, an easy diagram chase proves that $H\left(\bar{B}^{n}(\psi)\right)$ is natural with respect to $\pi$.

Remark 8.2. Let $\pi$ be an abelian group. The fact that the homology of the bar constructions $\bar{B}^{n} S\left(\pi \otimes_{\mathbb{Z}} \mathbb{k}\right)$ and $\bar{B}^{n} \mathbb{k} \pi$ are isomorphic is well known [DP, 4.16]. The fact that we can build an isomorphism natural with respect to $\pi$ is less known (and it is actually false if $\pi$ is not a free abelian group).

Let us denote for short by $H(n, \mathbb{k})$ the functor which sends a free abelian group $\pi$ to the graded algebra $H_{*}^{\text {sing }}(K(\pi, n), \mathbb{k})=H_{*}\left(\bar{B}^{n}(\mathbb{k} \pi)\right)$. Proposition 8.1 has the following consequence.

Corollary 8.3. Let $n$ be a nonnegative integer. If $\mathbb{k}=\mathbb{Z}$, there is an isomorphism of $\mathcal{F}_{\mathbb{Z}}$-graded algebras

$$
H\left(\bar{B}^{n} \Gamma[2]\right) \simeq H(n+2, \mathbb{Z}) .
$$

If $p$ is a prime and $\mathbb{k}=\mathbb{F}_{p}, H\left(n, \mathbb{F}_{p}\right)$ factors into a $\mathcal{F}_{\mathbb{F}_{p}}$-graded algebra $\bar{H}\left(n, \mathbb{F}_{p}\right)$ and we have an isomorphism of $\mathcal{F}_{\mathbb{F}_{p}}$-graded algebras

$$
H\left(\bar{B}^{n} \Gamma[2]\right) \simeq \bar{H}\left(n+2, \mathbb{F}_{p}\right) .
$$


Corollary 8.3 shows that we can recover the homology of $\bar{B}^{n}(\Gamma[2])$, as a $\mathcal{F}_{\mathbb{k}^{-}}$-graded algebra, when the ground ring $\mathbb{k}$ is $\mathbb{Z}$ or $\mathbb{F}_{p}$, from the algebraic topology computations of [Car]. But for our purposes, we need more. We need the $\mathcal{P}_{\mathbb{k}}$-graded algebra structure of the homology of $\bar{B}^{n}(\Gamma[2]$ ) (or at least the data of the weights) in order to be able to apply our regrading functors from section 6. We also wish to compute the homology of $\bar{B}^{n}(\Gamma[2])$ over an arbitrary ground field $\mathbb{k}$ (not just a prime field). So in this part of the paper, we elaborate on Cartan's results to obtain a more satisfactory answer to problem 1. Our first main result is theorem 10.14, which solves problem 1 when $\mathbb{k}$ is an arbitrary field. Our second main result is theorem 11.13, which describes $H_{*}\left(\bar{B}^{n}\left(\Gamma\left(\mathbb{Z}^{m}[2]\right)\right)\right)$ as a graded algebra with weights for $\mathbb{k}=\mathbb{Z}$. We deduce from these theorems an explicit computation of $\mathbb{E}(S, \Lambda)$ and $\mathbb{E}(S, \Gamma)$. Section 9 is a preparatory work for our first main result.

\section{Strict polynomial StruCtures on Divided POWER Algebras}

Let $\mathbb{k}$ be a field of prime characteristic. If a functor $F \in \mathcal{F}_{\mathbb{k}}$ is graded, we can form the $\mathcal{F}_{\mathbb{k}}$-graded algebra:

$$
U(F)=\Gamma\left(F_{\text {even }}\right) \otimes \Lambda\left(F_{\text {odd }}\right)
$$

We denote this algebra by $U(F)$ because it satisfies a universal property, see theorem 10.2 below. Recall from section 3 the forgetful functor

$$
\mathcal{U}:\left\{\mathcal{P}_{\mathbb{k}} \text {-graded alg. }\right\} \rightarrow\left\{\mathcal{F}_{\mathbb{k}^{k}} \text {-graded alg. }\right\} .
$$

In this section, we determine all the $\mathcal{P}_{\mathbb{R}^{-}}$-graded algebras $A$ such that $\mathcal{U} A=$ $U(F)$, if $F$ is additive. We also do the same for $\Gamma(F)$ in characteristic 2.

9.1. Strict polynomial structures on $U(F)$. We first need a few results about additive strict polynomial functors. We say that a functor $F \in \mathcal{P}_{\mathbb{k}}$ is additive if the underlying functor $\mathcal{U} F \in \mathcal{F}_{\mathbb{k}}$ is additive.

Lemma 9.1. Let $\mathbb{k}$ be a field of prime characteristic.

- (Classification) If $F \in \mathcal{P}_{\mathbb{k}}$ is additive with finite dimensional values, then $F$ either equals zero or is a finite direct sum of Frobenius twists $I^{(r)}$ (with possibly different $r \geq 0$ ).

- (Retracts) Let $F, G$ be additive functors with finite dimensional values, and let $f \in \operatorname{Hom}_{\mathcal{P}_{\mathrm{k}}}(F, G)$. Then $(i)$ and (ii) are equivalent.

(i) There exists $V \in \mathcal{V}_{\mathbb{k}}$ such that the $\mathbb{k}$-linear map $f_{V}: F(V) \rightarrow$ $G(V)$ is surjective

(ii) There exists $\iota \in \operatorname{Hom}_{\mathcal{P}_{\mathbb{k}}}(G, F)$ such that $f \circ \iota=\operatorname{Id}_{F}$.

Proof. To prove the classification, we can assume that $F$ is homogeneous of degree $d$. If $d=0$, then $F$ is constant and additive, hence $F=0$. So let us assume that $d \geq 1$. There are two cases.

Case 1: $d$ is not a power of $p$. The map

$$
\begin{array}{ccc}
\operatorname{End}_{\mathbb{k}}(\mathbb{k}) & \rightarrow & \operatorname{End}_{\mathbb{k}}(F(\mathbb{k})) \\
f & \mapsto & F(f)
\end{array}
$$

is given by a homogeneous polynomial of degree $d$, which is additive. Since $d$ is not a power of $p$, the only such polynomial is the zero polynomial. Thus, $F$ sends the identity map of $\mathbb{k}$ to zero. So the identity map of $F(\mathbb{k})$ equals zero. Thus $F(\mathbb{k})=0$. By additivity of $F$ this implies that $F=0$. 
Case 2: $d=p^{r}$, for $r \geq 0$. Assume that $F \neq 0$ and fix an integer $n \geq p^{r}$. Recall from [FS, Cor 3.13] that evaluation on $\mathbb{k}^{n}$, yields an equivalence of categories $\mathcal{P}_{p^{r}, \mathbb{k}} \simeq S\left(n, p^{r}\right)-\bmod \left(\right.$ where $S\left(n, p^{r}\right)$ is the Schur algebra). So it suffices to prove that $F\left(\mathbb{k}^{n}\right)$ is a direct sum of copies of $\left(\mathbb{k}^{n}\right)^{(r)}$.

Let $\mathbb{k}_{i}$ denote the vector space $\mathbb{k}$ acted on by the torus $\mathbb{G}_{m}^{\times n}$ by $\left(\lambda_{1}, \ldots, \lambda_{n}\right)$. $x=\lambda_{i} \cdot x$. Since $F$ is homogeneous of degree $p^{r}, F\left(\mathbb{k}_{i}\right)$ is acted on by $\mathbb{G}_{m}^{\times n}$ by $\left(\lambda_{1}, \ldots, \lambda_{n}\right) \cdot x=\lambda_{i}^{p^{r}} \cdot x$. Additivity of $F$ yields a $\mathbb{G}_{m}^{\times n}$-equivariant isomorphism

$$
F\left(\mathbb{k}^{n}\right)=F\left(\bigoplus_{i \leq n} \mathbb{k}_{i}\right) \simeq \bigoplus_{i \leq n} F\left(\mathbb{k}_{i}\right) .
$$

As a consequence, all the weights of the $S\left(n, p^{r}\right)$-module $F\left(\mathbb{k}^{n}\right)$ are of the form $\left(\mu_{1}, \ldots, \mu_{n}\right)$ with all $\mu_{i}=0$ but one which equals $p^{r}$. In particular, if $S_{1}\left(\mathbb{k}^{n}\right), \ldots, S_{N}\left(\mathbb{k}^{n}\right)$ is the composition series of $F\left(\mathbb{k}^{n}\right)$, the $S_{i}\left(\mathbb{k}^{n}\right)$ are finite direct sums of simples with highest weight $\left(p^{r}, 0, \ldots, 0\right)$, that is of $\left(\mathbb{k}^{n}\right)^{(r)}$. We know (see e.g. [FS] ) that $\operatorname{Ext}^{1}\left(\left(\mathbb{k}^{n}\right)^{(r)},\left(\mathbb{k}^{n}\right)^{(r)}\right)=0$. Thus there cannot be nontrivial extensions between finite direct sums of $\left(\mathbb{k}^{n}\right)^{(r)}$. This implies that the composition series of $F\left(\mathbb{k}^{n}\right)$ has length $N=1$. Thus $F\left(\mathbb{k}^{n}\right)$ is a finite direct sum of copies of $\left(\mathbb{k}^{n}\right)^{(r)}$.

Finally, let us prove the characterization of retracts. We can assume that $F, G$ are homogeneous of degree $p^{r}, r \geq 1$. The identity map is a basis of $\operatorname{Hom}_{\mathcal{P}_{\mathrm{k}}}\left(I^{(r)}, I^{(r)}\right)$, so tensor products yield isomorphisms for $k, \ell \geq 1$ :

$$
\operatorname{Hom}_{\mathbb{k}}\left(\mathbb{k}^{k}, \mathbb{k}^{\ell}\right) \simeq \operatorname{Hom}_{\mathcal{P}_{\mathbb{k}}}\left(I^{(r)} \otimes \mathbb{k}^{k}, I^{(r)} \otimes \mathbb{k}^{\ell}\right), \quad f \mapsto \operatorname{Id} \otimes f,
$$

and the result follows.

If $A$ is an augmented $\mathcal{P}_{\mathbb{k}^{-}}$(or $\mathcal{F}_{\mathbb{k}^{-}}$) graded algebra, we denote by $Q(A)$ the indecomposables of $A$, that is $Q(A)$ is the cokernel of the multiplication $A^{\prime} \otimes A^{\prime} \rightarrow A^{\prime}$, where $A^{\prime}$ is the augmentation ideal of $A$. If $A$ is a $\mathcal{P}_{\mathbb{k}^{-}}$-graded augmented algebra, then $Q(A)$ is a graded strict polynomial functor and $\mathcal{U} Q(A)=Q(\mathcal{U} A)$. Similarly the primitives $P(C)$ of a $\mathcal{P}_{\mathbb{k}^{k}}$-graded coaugmented coalgebra $C$ form a graded strict polynomial subfunctor of $C$ and $\mathcal{U} P(C)=P(\mathcal{U} C)$. The following lemma explains the link between additive and exponential functors.

Lemma 9.2. Let $\mathbb{k}$ be a field, and let $E$ be an exponential functor. The graded strict polynomial functors $P(E)$ and $Q(E)$ are additive. Moreover, if there exists $V \in \mathcal{V}_{\mathbb{k}}$ such that the composite $P(E)(V) \hookrightarrow E(V) \rightarrow Q(E)(V)$ is surjective, then $Q(E)$ is a direct summand in $E$.

Proof. Let $E^{\prime}$ be the augmentation ideal of $E$. Since $E$ is exponential, we have $E^{\prime}(V \oplus W)$ is isomorphic to $E^{\prime}(V) \otimes \mathbb{k} \oplus \mathbb{k} \otimes E^{\prime}(W) \oplus E^{\prime}(V) \otimes E^{\prime}(W)$. Moreover, the multiplication $E^{\prime}(V \oplus W)^{\otimes 2} \rightarrow E^{\prime}(V \oplus W)$ identifies through this decomposition with the direct sum of three maps (which are induced by multiplications):

$$
\begin{aligned}
& \left(E^{\prime}(V) \otimes \mathbb{k}\right)^{\otimes 2} \rightarrow E^{\prime}(V) \otimes \mathbb{k}, \\
& \left(\mathbb{k} \otimes E^{\prime}(W)\right)^{\otimes 2} \rightarrow \mathbb{k} \otimes E^{\prime}(W), \\
& \left(E^{\prime}(V) \otimes \mathbb{k}\right) \otimes\left(\mathbb{k} \otimes E^{\prime}(W)\right) \oplus \begin{array}{l}
\text { other summands } \\
\text { of } E^{\prime}(V \oplus W)^{\otimes 2}
\end{array} \rightarrow E^{\prime}(V) \otimes E^{\prime}(W) .
\end{aligned}
$$


The first two maps have respective cokernels $Q(E)(V)$ and $Q(E)(W)$ and the last one is surjective. This shows that $Q(E)$ is additive. The proof that the primitives are additive is similar.

Finally, if the map $P(E)(V) \rightarrow Q(E)(V)$ is surjective for some $V \in \mathcal{V}_{\mathbb{k}}$, then by lemma 9.1, it admits a section $\iota$. So the composite $Q(E) \stackrel{\iota}{\rightarrow} P(E) \hookrightarrow$ $E$ is a section of $E \rightarrow Q(E)$.

We are now ready to prove the main result of section 9 ,

Theorem 9.3. Let $\mathbb{k}$ be a field of prime characteristic $p$, and for all $d \geq 0$, let $F_{d} \in \mathcal{F}_{\mathrm{k}}$ be a finite direct sum of $n_{d}$ copies of the identity functor, and let $F=\bigoplus_{d \geq 0} F_{d}$ The graded $\mathcal{P}_{\mathbb{k}}$-algebras $A$ satisfying $\mathcal{U} A=U(F)$ are of the form:

$$
A=\Gamma\left(G_{\text {even }}\right) \otimes \Lambda\left(G_{\text {odd }}\right),
$$

where $G=\bigoplus_{d \geq 0} G_{d}$ and each $G_{d} \in \mathcal{P}_{\mathbb{k}}$ is a direct sum of $n_{d}$ Frobenius twists $I^{(r)}$ (with possibly different $r \geq 0$ ).

Proof. Step 1: Duality. Let $E$ be a graded exponential (non strict polynomial) functor. Then finding the $\mathcal{P}_{\mathbb{k}}$-graded algebras $A$ such that $\mathcal{U} A=E$ is equivalent to finding the $\mathcal{P}_{\mathbb{k}}$-graded algebras $B$ such that $\mathcal{U} B=E^{\sharp}$ as algebras. Indeed, if $\mathcal{U} A=E$, then for all $V, W \in \mathcal{V}_{\mathbb{k}}$ the composite

$$
A(V) \otimes A(W) \rightarrow A(V \oplus W)^{\otimes 2} \rightarrow A(V \oplus W)
$$

is an isomorphism (indeed, this is true for $\mathcal{U} A=E$, and the forgetful functor $\mathcal{U}$ reflects isomorphisms). Thus $A$ is an exponential functor, and $\mathcal{U} A$ coincides with $E$ as an exponential functor. Equivalently, $\mathcal{U}\left(A^{\sharp}\right)$ coincides with $E^{\sharp}$ as an exponential functor. This is in turn equivalent to the fact that $B=A^{\sharp}$ is a graded strict polynomial algebra such that $\mathcal{U} B$ coincides with $E^{\sharp}$ as a $\mathcal{F}_{\mathbb{k}^{k}}$-graded algebra.

So, to prove theorem 9.3 , it suffices to prove that the graded strict polynomial algebras $B$ such that $\mathcal{U} B=S\left(F_{\text {even }}\right) \otimes \Lambda\left(F_{\text {odd }}\right)$ are of the form $S\left(G_{\text {even }}\right) \otimes \Lambda\left(G_{\text {odd }}\right)$ with $G$ as indicated.

Step 2: Indecomposables. If $B$ is as indicated in step 1, then the indecomposables of $B$ are a direct summand in $B$. Indeed, since $\mathcal{U} B=$ $S\left(F_{\text {even }}\right) \otimes \Lambda\left(F_{\text {odd }}\right)$, there exists $V \in \mathcal{V}_{\mathbb{k}}$, e.g. $V=\mathbb{k}$, such that the composite $P(B)(V) \rightarrow B(V) \rightarrow Q(B)(V)$ is surjective. Then one applies lemma 9.2 .

Now, the indecomposables of $B$ is an additive strict polynomial functor $Q(B)$ satisfying $\mathcal{U} Q(B)=Q(\mathcal{U} B)=F$. So by lemma 9.1, $Q(B)_{d}$ is a finite direct sum of $n_{d}$ Frobenius twists for all $d \geq 0$.

Step 3: Universal property. The morphism of graded strict polynomial functors $Q(B) \hookrightarrow B$ induces a morphism of $\mathcal{P}_{\mathbb{k}}$-graded algebras $S\left(Q(B)_{\text {even }}\right) \otimes \Lambda\left(Q(B)_{\text {odd }}\right) \rightarrow B$. For all $V \in \mathcal{V}_{\mathbb{k}}$, this morphism is an isomorphism after evaluation on $V$. Hence, it is an isomorphism. Thus $B$ is of the form $S\left(G_{\text {even }}\right) \otimes \Lambda\left(G_{\text {odd }}\right)$ with $G=Q(B)$ as indicated in the statement of theorem 9.3, which concludes the proof.

9.2. Strict polynomial structures on $\Gamma(F)$. Now we work over a field $\mathbb{k}$ of characteristic 2 . If $F$ is a graded strict polynomial functor, then the symmetric algebra $S(F)$ is commutative, so we can adapt the proof of theorem 
9.3 to get the following result (the commutativity of $S(F)$ is needed in the last step of the proof, the remainder of the proof is unchanged).

Theorem 9.4. Let $\mathbb{k}$ be a field of characteristic 2 , and for all $d \geq 0$, let $F_{d} \in \mathcal{F}_{\mathbb{k}}$ be a finite direct sum of $n_{d}$ copies of the identity functor, and let $F:=\bigoplus_{d \geq 0} F_{d}$. The graded $\mathcal{P}_{\mathbb{k}}$-algebras $A$ satisfying $\mathcal{U} A=\Gamma(F)$ are of the form: $A=\Gamma(G)$, where $G=\bigoplus_{d \geq 0} G_{d}$ and each $G_{d} \in \mathcal{P}_{\mathbb{k}}$ is a direct sum of $n_{d}$ Frobenius twists $I^{(r)}$ (with possibly different $r \geq 0$ ).

\section{EXPLicit COMPUTATIONS OVER A FIELD}

10.1. Systems of divided powers on an algebra. In this section, $\mathbb{k}$ is a commutative ring. We recall the basics of systems of divided powers. The reader can take [Car, Exp. 7, 8], [Eis, Appendix 2] as references.

Definition 10.1. Let $A$ be a graded commutative $\mathbb{k}$-algebra. A system of divided powers on $A$ is a set of maps $\left(\gamma_{r}\right)_{r \geq 0}$ defined over the even degree part of $A$, and satisfying the following axioms:

(a) $\gamma_{0}(x)=1, \gamma_{1}(x)=x$ and $\gamma_{k}$ maps $A_{i}$ into $A_{k i}$ for $i \geq 2$.

(b) $\gamma_{k}(x) \gamma_{\ell}(x)=\left(\begin{array}{c}k+\ell \\ k\end{array}\right) \gamma_{k+\ell}(x)$.

(c) $\gamma_{k}(x+y)=\sum_{i+j=k} \gamma_{i}(x) \gamma_{j}(y)$.

(d) $\gamma_{k}(x y)=0$ if $k \geq 2$ and $x$ and $y$ have odd degrees, $=x^{k} \gamma_{k}(y)$ if $k \geq 2$ and $|x| \geq 2$ is even and $|y|$ is even.

(e) $\gamma_{\ell}\left(\gamma_{k}(x)\right)=\frac{(k \ell) !}{\ell !(k !)^{\ell}} \gamma_{k \ell}(x)$.

A morphism of algebras $f: A \rightarrow B$ preserves divided powers if $\gamma_{k}(f(x))=$ $f\left(\gamma_{k}(x)\right)$ for all $k$.

Observe that the maps $\gamma_{r}$ are not $\mathbb{k}$-linear: actually, equation (b) implies that $k ! \gamma_{k}(x)=x^{k}$, which is the reason for the name 'divided powers'.

Theorem 10.2 ([Car, Exp 8, Section 4]). Let $V$ be a graded free $\mathbb{k}$-module. There exists a unique system of divided powers on the graded algebra $U(V)=$ $\Gamma\left(V_{\text {even }}\right) \otimes \Lambda\left(V_{\text {odd }}\right)$ such that $\gamma_{k}(x)=x^{\otimes k}$ for $x \in V_{\text {even }}$ and $k \geq 0$.

Moreover, for all commutative graded $\mathbb{k}$-algebra $A$ equipped with divided powers, and all graded $\mathbb{k}$-linear map $f: V \rightarrow A$, there exists a unique morphism of algebras $\bar{f}: U(V) \rightarrow A$ extending $f$ and preserving divided powers.

Theorem 10.2 is a mean to construct morphisms of algebras with a very big image (compare with the image of the map induced by the universal property of the free graded commutative algebra on $V$ ). However, theorem 10.2 is not so efficient in characteristic 2 . For example, the graded $\mathbb{k}$-algebra $\Gamma(V)$ is graded commutative even if $V$ is concentrated in odd degree, and in that case, theorem 10.2 yields a morphism: $\Lambda\left(V_{\text {odd }}\right) \rightarrow \Gamma\left(V_{\text {odd }}\right)$ with quite a small image. To bypass this problem, there is a stronger notion of divided powers for strictly anticommutative (i.e. graded commutative with $x^{2}=0$ if $x$ has odd degree) algebras in characteristic 2. To avoid confusion, we call this notion 'strong divided powers', although this notion is simply called 'divided powers' in the literature. 
Definition 10.3. Let $A$ be a strictly anticommutative graded $\mathbb{k}$-algebra, for $\mathbb{k}$ of characteristic 2. A system of strong divided powers is a collection of maps $\left(\gamma_{r}\right)_{r \geq 0}$ defined over the part of positive degree of $A$ and satisfying equations $(a)-(e)$ above.

With this stronger notion of divided powers, theorem 10.2 becomes $\mathrm{Car}$, Exp 8, Thm 2 bis]:

Theorem 10.4 ([Car $)$. Let $\mathbb{k}$ be a ring of characteristic 2 . Let $V$ be a graded free $\mathbb{k}$-module. There exists a unique system of strong divided powers on the graded algebra $\Gamma(V)$ such that $\gamma_{k}(x)=x^{\otimes k}$ for $x \in V$ and $k \geq 0$.

Moreover, for all strictly anticommutative graded $\mathbb{k}$-algebra $A$ equipped with strong divided powers, and all graded $\mathbb{k}$-linear map $f: V \rightarrow A$, there exists a unique morphism of algebras $\bar{f}: U(V) \rightarrow A$ extending $f$ and preserving divided powers.

10.2. Homology operations in bar constructions. Let $\mathbb{k}$ be a commutative ring, and let $A$ be a cdg-㑊-algebra. The homology of $\bar{B}^{n} A$ is equipped with the following homology operations, natural with respect to $A$.

(1) Suspension. For all $n \geq 0$ and all $k \geq 0$, there is a $\mathbb{k}$-linear suspension operation [Car, Exp 6, sections 1 and 2]

$$
\sigma: H_{k}\left(\bar{B}^{n} A\right) \rightarrow H_{k+1}\left(\bar{B}^{n+1} A\right) .
$$

(2) Transpotence. Assume $\mathbb{k}$ has prime characteristic $p \neq 2$. Then for all $n \geq 1$ and all $k \geq 1$ there is an additive (hence $\mathbb{k}$-linear if $\mathbb{k}=\mathbb{F}_{p}$ ) transpotence operation [Car, Exp 6, section 4]

$$
\phi_{p}: H_{2 k}\left(\bar{B}^{n} A\right) \rightarrow H_{p 2 k+2}\left(\bar{B}^{n+1} A\right) .
$$

(3) Divided powers. Assume that $A$ is strictly anticommutative 4 (i.e. $a^{2}=0$ for $a$ of odd degree). Then for all $n \geq 1$, there is a system of divided powers $\left(\gamma_{r}\right)_{r \geq 0}$ on $H\left(\bar{B}^{n} A\right)$ Car, Exp 7, Thm 1 and section 5]. If $\mathbb{k}$ has characteristic 2 , there is a system of strong divided powers on $H\left(\bar{B}^{n} A\right)$ [Car, Exp 7, section 8].

In our computation of $\bar{B}(\Gamma[2])$ in theorem 10.14, we shall need the following complement on homological operations.

Proposition 10.5. Let $\mathbb{k}$ be a commutative ring, and let $A$ be a cdg-kalgebra with weights. The homology of $\bar{B}^{n}(A)$ is a wg-k-algebra, and the homological operations have the following behavior with respect to weights. The suspension preserves the weights. The transpotence $\phi_{p}$ multiplies the weights by $p$, and the divided power operation $\gamma_{r}$ multiplies the weights by $r$.

Proof. To prove proposition 10.5, we have to go back to the definition of the homology operations. Let us denote by $C$ the wdg-倓-algebra $C=\bar{B}^{n} A$. Let us first treat the case of the suspension. It is defined on the chain level by the map $C \hookrightarrow \bar{B} C c \mapsto[c]$. So it preserves the weights.

The cases of the transpotence and divided powers are slightly more involved. Recall from [Car, Exposes 3 et 4] that there is a wdg-k-algebra $B C$ characterized by the following properties.

\footnotetext{
${ }^{4}$ This applies to $\bar{B} A$, for $A$ graded commutative
} 
(i) $B C:=C \otimes \bar{B} C$ as a graded algebra with weights. Thus, both $C$ and $\bar{B} C$ can be viewed as weighted graded subalgebras of $B C$.

(ii) $B C$ is equipped with a weight-preserving differential $\partial$, such that following maps are morphisms of wdg- $\mathbb{k}$-algebras:

$$
C \stackrel{\mathrm{Id} \otimes 1}{\longrightarrow} B C, \quad B C \stackrel{\epsilon \otimes \mathrm{Id}}{\longrightarrow} \bar{B} C .
$$

(B) For all $k \geq 1$, the composite $\bar{B} C_{k+1} \hookrightarrow B C_{k+1} \stackrel{\partial}{\rightarrow} B C_{k}$ is injective and induces an isomorphism $\bar{B} C_{k+1} \simeq Z(B C)_{k}$ onto the cycles of degree $k$ of $B C$ (in particular, $H_{i}(B C)=0$ for $i>0$ ).

The transpotence $\phi_{p}$ is defined on the chain level as follows [Car, p. 605]. Let $c \in Z C_{2 k} \subset Z(B C)_{2 k}$ be a cycle representing a cohomology class $\alpha \in H_{2 k}(C)$. There is an $x \in \bar{B} C_{2 k}$ such that $\partial x=c$. Then there is an element $y \in Z(\bar{B} C)_{p 2 k+2}$ representing $\phi_{p}(\alpha)$ which satisfies $\partial y=c^{p-1} * \partial x$ (* denotes the product in $B C$ ). So the weight $w\left(\phi_{p}(\alpha)\right)$ of $\phi_{p}(\alpha)$ satisfies:

$$
w\left(\phi_{p}(\alpha)\right)=w(y)=w\left(c^{p-1}\right)+w(x)=p w(c)=p w(\alpha) .
$$

The divided powers are defined on the chain level $\bar{B} C$ by [Car, Exp 7, proof of Thm 1]: $\gamma_{1}(c)=c$ and $\partial \gamma_{r}(c)=\partial c * \gamma_{r-1}(c)$ for $r \geq 1$. Since $\partial$ preserves the weights $w\left(\gamma_{r}(c)\right)=w(c)+w\left(\gamma_{r-1}(c)\right)$ for $r \geq 1$ and the result follows by induction on $r$.

10.3. Cartan's result. We are now ready to state Cartan's computation of $H_{*}^{\text {sing }}\left(K(\pi, n), \mathbb{F}_{p}\right)$. In view of corollary 8.3 , we state the results in terms of the homology of $\bar{B}^{n}(\Gamma[2])$. Let us assume first that $p \neq 2$.

Definition 10.6. A p-admissible word 5 is a finite sequence $\underline{w}$ of letters $\sigma$, $\phi_{p}$ and $\gamma_{p}$ starting on the left with the letter $\sigma$ or $\phi_{p}$, and finishing on the right with the letter $\sigma$, satisfying the following property. For all letter $\phi_{p}$ or $\gamma_{p}$ of $\underline{w}$, the number of $\sigma$ on the right of the letter is even. The height of a word $\underline{w}$ is the number of letters equal to $\sigma$ or $\phi_{p}$ in $\underline{w}$. The degree $\operatorname{deg}(\underline{w})$ of a word $\underline{w}$ is defined recursively by $\operatorname{deg}(\alpha)=0$ if $\alpha$ is empty, and

$\operatorname{deg}(\sigma \alpha)=1+\operatorname{deg}(\alpha), \quad \operatorname{deg}\left(\gamma_{p} \alpha\right)=p \operatorname{deg}(\alpha), \quad \operatorname{deg}\left(\phi_{p} \alpha\right)=p \operatorname{deg}(\alpha)+2$.

Example 10.7. The $p$-admissible words of height 3 are the words:

$$
\sigma \gamma_{p}^{k} \sigma \sigma, k \geq 0, \quad \text { and } \quad \phi_{p} \gamma_{p}^{k} \sigma \sigma, k \geq 0 .
$$

(By convention $\gamma_{p}^{k}$ is empty if $k=0$ ). Moreover, $\operatorname{deg}\left(\sigma \gamma_{p}^{k} \sigma \sigma\right)=2 p^{k}+1$ and $\operatorname{deg}\left(\phi_{p} \gamma_{p}^{k} \sigma \sigma\right)=2 p^{k+1}+2$. The $p$-admissible words of height 4 are the words:

$$
\sigma \sigma \gamma_{p}^{k} \sigma \sigma, \sigma \gamma_{p}^{k} \phi_{p} \gamma_{p}^{\ell} \sigma \sigma, \text { and } \phi_{p} \gamma_{p}^{k} \phi_{p} \gamma_{p}^{\ell} \sigma \sigma, \text { for } k, \ell \geq 0
$$

of respective degrees $2 p^{k}+2,2 p^{k+\ell+1}+2 p^{k}+1$ and $2 p^{k+\ell+2}+2 p^{k+1}+2$.

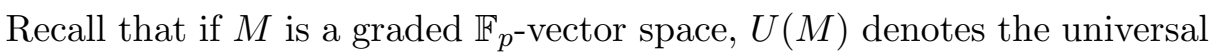
divided power algebra over $M$, i.e. $U(M)=\Gamma\left(M_{\text {even }}\right) \otimes \Lambda\left(M_{\text {odd }}\right)$.

\footnotetext{
${ }^{5}$ Such words are called 'mots admissibles de première espèce' in [Car, Exp 9, section 1]. We drop the words 'première espèce' in the definition since we shall not need the 'mots admissibles de deuxième espèce' in the article.
} 
Theorem 10.8 ([Car, Exp 9, théorème fondamental]). Let $p$ be an odd prime. For all $n \geq 0$, there is an isomorphism of graded $\mathcal{F}_{\mathbb{F}_{p}}$-algebras

$$
H\left(\bar{B}^{n}(\Gamma[2])\right) \simeq U\left(\bigoplus_{\underline{w}} I[\operatorname{deg}(\underline{w})]\right),
$$

where the direct sum on the right hand side is taken over all p-admissible words $\underline{w}$ of height $n+2$ and $I[\operatorname{deg}(\underline{w})]$ means a copy of the identity functor $I: \mathcal{V}_{\mathbb{F}_{p}} \rightarrow \mathcal{V}_{\mathbb{F}_{p}}$ placed in homological degree $\operatorname{deg}(\underline{w})$.

Remark 10.9. Let $\Gamma_{\mathbb{k}}$ denote the divided power algebra over a field $\mathbb{k}$. For all $\mathbb{F}_{p}$-module $V$ there is an isomorphism of graded $\mathbb{k}$-algebras $\Gamma_{\mathbb{F}_{p}}(V[2]) \otimes_{\mathbb{F}_{p}}$ $\mathbb{k} \simeq \Gamma_{\mathbb{k}}\left(V \otimes_{\mathbb{F}_{p}} \mathbb{k}[2]\right)$, hence an isomorphism of graded $\mathbb{k}$-algebras:

$$
H\left(\bar{B}^{n}\left(\Gamma_{\mathbb{F}_{p}}(V[2])\right)\right) \otimes_{\mathbb{F}_{p}} \mathbb{k} \simeq H\left(\bar{B}^{n}\left(\Gamma_{\mathbb{F}_{p}}\left(V \otimes_{\mathbb{F}_{p}} \mathbb{k}[2]\right)\right)\right) .
$$

Observe that the isomorphism above is natural with respect to the $\mathbb{F}_{p}$-vector space $V$ but does not tell us anything about the functoriality with respect to the $\mathbb{k}$-vector space $V \otimes_{\mathbb{F}_{p}} \mathbb{k}$. So we cannot deduce the description of the homology of iterated bar constructions over a general field $\mathbb{k}$, as a graded $\mathcal{F}_{\mathbb{k}}$-algebra, from theorem 10.8 ,

Actually, Cartan's result is more precise. It says that each copy of the identity functor on the right hand side is simply obtained by applying a suitable sequence of homology operations to $I[2]=(\Gamma[2])_{2}=H_{2}\left(\bar{B}^{2} S\right)$. For example, if $n=2$, the copy of $I$ corresponding to the word $\sigma \phi_{p} \gamma_{p}^{3} \sigma \sigma$ is the image of $I[2]$ by the sequence of operations:

$$
(\Gamma[2])_{2} \stackrel{\gamma_{p}^{3}}{\longrightarrow}(\Gamma[2])_{2 p^{3}} \stackrel{\phi_{p}}{\longrightarrow} H_{2 p^{4}+2}(\bar{B}(\Gamma[2])) \stackrel{\sigma}{\rightarrow} H_{2 p^{4}+3}\left(\bar{B}^{2}(\Gamma[2])\right) .
$$

In general, the operations to be applied are the one needed to complete the final letters ' $\sigma \sigma$ ' in order to obtain the word indexing the copy of the identity functor $I$ considered, starting from the right to the left. This produces a morphism of graded functors:

$$
\bigoplus_{\underline{w}} I[\operatorname{deg}(\underline{w})] \rightarrow H\left(\bar{B}^{n}(\Gamma[2])\right)
$$

and the isomorphism of theorem 10.8 is produced from this morphism and the universal property of $U$.

Let us now describe the case $p=2$. This case is actually simpler. The definition of admissible words is modified as follows.

Definition 10.10. A 2-admissible word is a finite sequence $\underline{w}$ of letters $\sigma$, and $\gamma_{2}$ starting with the letter $\sigma$ and finishing with the letters $\sigma \sigma$. The height of a word $\underline{w}$ is the number of $\sigma$ in $\underline{w}$. Its degree $\operatorname{deg}(\underline{w})$ is defined recursively by $\operatorname{deg}(\alpha)=0$ if $\alpha$ is empty, and $\operatorname{deg}(\sigma \alpha)=1+\operatorname{deg}(\alpha)$ and $\operatorname{deg}\left(\gamma_{2} \alpha\right)=2 \operatorname{deg}(\alpha)$.

Example 10.11. The 2-admissible words of height 3 are the words $\sigma \gamma_{2}^{k} \sigma \sigma$ with $k \geq 0$, and $\operatorname{deg}\left(\sigma \gamma_{2}^{k} \sigma \sigma\right)=2^{k+1}+1$. The 2 -admissible words of height 4 are the words $\sigma \gamma_{2}^{k} \sigma \gamma_{2}^{\ell} \sigma \sigma$ for $k, \ell \geq 0$ of degree $2^{k+\ell+1}+2^{k}+1$.

The analogue of theorem 10.8 over $\mathbb{F}_{2}$ takes the following form Car, Exp 10, théorème fondamental]. 
Theorem 10.12 (融). For all $n \geq 0$, there is an isomorphism of graded $\mathcal{F}_{\mathbb{F}_{2}}$-algebras

$$
H\left(\bar{B}^{n}(\Gamma[2])\right) \simeq \Gamma\left(\bigoplus_{\underline{w}} I[\operatorname{deg}(\underline{w})]\right),
$$

where the direct sum on the right hand side is taken over all 2-admissible words $\underline{w}$ of height $n+2$ and $I[\operatorname{deg}(\underline{w})]$ means a copy of the identity functor $I: \mathcal{V}_{\mathbb{F}_{2}} \rightarrow \mathcal{V}_{\mathbb{F}_{2}}$ placed in homological degree $\operatorname{deg}(\underline{w})$.

10.4. Homology of $\bar{B}^{n}(\Gamma[2])$ over an arbitrary field. We are now ready to prove the main result of section 10, namely the computation of the homology of $\bar{B}^{n}(\Gamma[2])$, as a strict polynomial algebra, and over an arbitrary field $\mathbb{k}$. We first need to introduce a definition.

Definition 10.13. Let $\underline{w}$ be a $p$-admissible word ( $p$ even or odd). The twisting of a $p$-admissible word $\underline{w}$ is the number of letters equal to $\phi_{p}$ or $\gamma_{p}$ in $\underline{w}$. We denote the twisting of $\underline{w}$ by $t_{\underline{w}}$.

Theorem 10.14. Let $\mathbb{k}$ be a field of positive characteristic $p$. If $p$ is odd, we have an isomorphism of graded $\mathcal{P}_{\mathbb{k}}$-algebras:

$$
H\left(\bar{B}^{n}(\Gamma[2])\right) \simeq U\left(\bigoplus_{\underline{w}} I^{\left(t_{\underline{w}}\right)}[\operatorname{deg}(\underline{w})]\right),
$$

where the sum is taken over all p-admissible words $\underline{w}$ of height $n+2$, and $I^{\left(t_{\underline{w}}\right)}[\operatorname{deg}(\underline{w})]$ denotes a copy of the $t_{\underline{w}}$ th Frobenius twist functor, placed in homological degree $\operatorname{deg}(\underline{w})$. If $p=\overline{2}$ we have an isomorphism of graded $\mathcal{P}_{\mathbb{k}}$-algebras:

$$
H\left(\bar{B}^{n}(\Gamma[2])\right) \simeq \Gamma\left(\bigoplus_{\underline{w}} I^{\left(t_{\underline{w}}\right)}[\operatorname{deg}(\underline{w})]\right),
$$

where the sum is taken over all 2-admissible words $\underline{w}$ of height $n+2$.

Proof. Let us prove the case $p$ odd (the case $p=2$ is similar). We start with the case $\mathbb{k}=\mathbb{F}_{p}$. By theorem 10.8, the graded $\mathcal{F}_{\mathbb{F}_{p}}$-algebra $\mathcal{U} H\left(\bar{B}^{n}(\Gamma[2])\right)$ is isomorphic to $U\left(\bigoplus_{\underline{w}} I[\operatorname{deg}(\underline{w})]\right)$. Hence by theorem $\left[9.3\right.$, the graded $\mathcal{P}_{\mathbb{F}_{p}}$ algebra $H\left(\bar{B}^{n}(\Gamma[2])\right)$ is of the form $U\left(\bigoplus_{\underline{w}} I^{\left(r_{\underline{w}}\right)}[\operatorname{deg}(\underline{w})]\right)$, where the $r_{\underline{w}}$ are nonnegative integers which we have to determine. Actually, these integers are given by the weights of the $H\left(\bar{B}^{n}(\Gamma[2])\right)$. Let $V \in \mathcal{V}_{\mathbb{F}_{p}}$. By proposition 10.5 the morphism induced by the cohomology operations

$$
U\left(\bigoplus_{\underline{w}} V[\operatorname{deg}(\underline{w})]\right) \rightarrow H\left(\bar{B}^{n} \Gamma(V[2])\right)
$$

becomes a morphism of weighted graded $\mathbb{F}_{p}$-algebras if we let the copy of $V$ indexed by $\underline{w}$ have weight $t_{\underline{w}}$. Hence, the weighted graded algebra structure of $H\left(\bar{B}^{n}(\Gamma[2])\right)$ implies that $r_{\underline{w}}=t_{\underline{w}}$.

To get the result for all fields $\mathbb{k}$ it suffices to use the exact base change for strict polynomial functors (Notice: this feature is specific to strict polynomial functors!). If $\mathbb{k}$ is a field of characteristic $p$, there is an exact functor [SFB, Prop 2.6]: $\otimes_{\mathbb{F}_{p} \mathbb{k}}: \mathcal{P}_{\mathbb{F}_{p}} \rightarrow \mathcal{P}_{\mathbb{k}}$. This base change functor commutes with tensor products and sends divided powers to divided powers and Frobenius twists to Frobenius twists. Whence the result. 
Remark 10.15. At first sight, the result for $p=2$ seems very different from the result for $p$ odd. However, if $p=2$ and $V \in \mathcal{V}_{\mathbb{F}_{2}}$, there is an isomorphism of graded algebras with weights (not natural with respect to $V$ ):

$$
\Gamma\left(V^{(r)}[i]\right) \simeq \Lambda\left(V^{(r)}[i]\right) \otimes \Gamma\left(V^{(r+1)}[2 i]\right)
$$

Indeed, we can prove it if $V=\mathbb{F}_{2}$ by direct inspection, and get the general result by the exponential formula.

10.5. Ext-computations over a field. Combining theorem 10.14 and theorem [7.5, we obtain the following computations of the $\mathcal{P}_{\mathbb{k}}$-graded algebras $\mathbb{E}(S, \Gamma)$ and $\mathbb{E}(S, \Lambda)$. Comparison with earlier computations of these algebras by other authors is made in section 15.3 .

Theorem 10.16. Let $\mathbb{k}$ be a field of odd characteristic $p$, and let $V$ be a finite dimensional $\mathbb{k}$-vector space. Let us denote by $I^{(k)}\langle i\rangle$ a copy of the $k$ th Frobenius twist functor, placed in cohomological degree $i$ (thus, $I^{(k)}\langle i\rangle=$ $\left.I^{(k)}[-i]\right)$. We have an isomorphism of $\mathcal{P}_{\mathbb{k}}$-graded algebras:

$$
\mathbb{E}(S, \Lambda) \simeq \Lambda\left(\bigoplus_{k \geq 0} I^{(k)}\left\langle p^{k}-1\right\rangle\right) \otimes^{1 \mathrm{t}} \Gamma\left(\bigoplus_{k \geq 0} I^{(k+1)}\left\langle p^{k+1}-2\right\rangle\right)
$$

where $A \otimes^{1} B$ denotes the 'signed tensor product' of two $\mathcal{P}_{\mathbb{k}}$-graded algebras and ${ }^{\mathrm{t}} A$ denotes the weight twisted algebra associated to $A$, as defined in section 4.3. We also have an isomorphism of $\mathcal{P}_{\mathbb{k}}$-graded algebras:

$$
\begin{aligned}
& \mathbb{E}(S, \Gamma) \simeq \\
& \Gamma\left(\bigoplus_{k \geq 0} V^{(k)}\left\langle 2 p^{k}-2\right\rangle\right) \otimes \Lambda\left(\bigoplus_{k \geq 0, \ell \geq 0} V^{(k+\ell+1)}\left\langle 2 p^{k+\ell+1}-2 p^{k}-1\right\rangle\right) \\
& \otimes \Gamma\left(\bigoplus_{k \geq 0, \ell \geq 0} V^{(k+\ell+2)}\left\langle 2 p^{k+\ell+2}-2 p^{k+1}-2\right\rangle\right) .
\end{aligned}
$$

Proof. Let us prove the first isomorphism, the second one is similar. Theorem 7.5 yields an isomorphism between $\mathbb{E}(S, \Lambda)$ and ${ }^{\mathrm{t}} \mathcal{R}_{3}(H \bar{B}(\Gamma[2]))$. The homology of $\bar{B}(\Gamma[2])$ is computed in theorem [10.14, and applying the computation rules for $\mathcal{R}_{\alpha}$ developed in section [6, we obtain

$$
\begin{aligned}
{ }^{t} \mathbb{E}(S, \Lambda) & =\mathcal{R}_{3} \Lambda\left(\bigoplus_{k \geq 0} I^{(k)}\left[2 p^{k}+1\right]\right) \otimes \mathcal{R}^{1} \mathcal{R}_{3} \Gamma\left(\bigoplus_{k \geq 0} I^{(k+1)}\left[2 p^{k+1}+2\right]\right), \\
& ={ }^{t} \Lambda\left(\bigoplus_{k \geq 0} I^{(k)}\left[-p^{k}+1\right]\right) \otimes{ }^{1} \Gamma\left(\bigoplus_{k \geq 0} I^{(k+1)}\left[-p^{k+1}+2\right]\right) .
\end{aligned}
$$

Using $I^{(k)}[-i]=I^{(k)}\langle i\rangle$, we obtain the result.

Theorem 10.17. Let $\mathbb{k}$ be a field of characteristic $p=2$, and let $V$ be a finite dimensional $\mathbb{k}$-vector space. Let us denote by $I^{(k)}\langle i\rangle$ a copy of the $k$-th Frobenius twist functor, placed in cohomological degree $i$. We have 
isomorphisms of $\mathcal{P}_{\mathbb{k}}$-graded algebras:

$$
\begin{aligned}
& \mathbb{E}(S, \Lambda) \simeq \Gamma\left(\bigoplus_{k \geq 0} I^{(k)}\left\langle p^{k}-1\right\rangle\right), \\
& \mathbb{E}(S, \Gamma) \simeq \Gamma\left(\bigoplus_{k \geq 0, \ell \geq 0} I^{(k+\ell)}\left\langle 2 p^{k+\ell}-p^{k}-1\right\rangle\right) .
\end{aligned}
$$

\section{Explicit COMPutations OVER the integers}

In this section, we work over the ground ring $\mathbb{k}=\mathbb{Z}$. We elaborate on Cartan's computation of the homology of EML-spaces with integral coefficients to compute the homology of $\bar{B}^{n}(\Gamma[2])$. Actually, Cartan made two descriptions of the integral homology of EML spaces $K(\pi, n)$ : a compact description, which is not natural with respect to $\pi$ (this is [Car, Exp 11, théorème 1]), and a description by generators and relations, which is natural with respect to $\pi$, but unfortunately quite complicated (this is Car, Exp 11, théorème 6]). For the sake of simplicity, we have chosen to use the compact description of the homology of EML spaces, therefore we only compute the homology of $\bar{B}^{n} \Gamma\left(\mathbb{Z}^{m}[2]\right)$ as graded algebras with weights. As a corollary, we compute $\mathbb{E}(S, \Lambda)\left(\mathbb{Z}^{m}\right)$ and $\mathbb{E}(S, \Gamma)\left(\mathbb{Z}^{m}\right)$ as wdg-Z-Zlgebras.

11.1. Dual Koszul and De Rham algebras. If $V$ is a graded $\mathbb{Z}$-module, we denote by $V[j]$ its homological suspension, that is, $V[j]_{i}:=V_{i-j}$.

Definition 11.1 (Dual Koszul algebra). Let $V$ be a positively graded $\mathbb{Z}$-free module concentrated in odd degrees. Then $\Gamma(V[1]) \otimes \Lambda(V)$ is a commutative graded-Z-Z-algebra. If $h$ is a positive integer, we define a differential $d_{K}$ as the composite:

$$
\begin{aligned}
\Gamma^{n}(V[1]) \otimes \Lambda^{k}(V) & \stackrel{\Delta \otimes \mathrm{Id}}{\longrightarrow} \Gamma^{n-1}(V[1]) \otimes V[1] \otimes \Lambda^{k}(V) \\
& \stackrel{h \mathrm{Id}}{\longrightarrow} \Gamma^{n-1}(V[1]) \otimes V \otimes \Lambda^{k}(V) \stackrel{\mathrm{Id} \otimes m}{\longrightarrow} \Gamma^{n-1}(V[1]) \otimes \Lambda^{k+1}(V) .
\end{aligned}
$$

This makes $\left(\Gamma(V[1]) \otimes \Lambda(V), d_{K}\right)$ into a commutative differential graded algebra which we call the 'dual Koszul algebra'. We denote it by $K^{h}(V)$.

If $h=1, K^{h}(V)$ is the graded dual of the usual Koszul algebra, see e.g. [FFSS, Section 4], whence the name. If $V$ is a copy of $\mathbb{Z}$ in odd degree, then $K^{h}(V)$ is nothing but an elementary complex of type (II) from [Car, Exp 11]. Dual Koszul algebras satisfy an exponential property: the following composite (where the first map is induced by the canonical inclusions into $V \oplus W)$ is an isomorphism of differential graded algebras

$$
K^{h}(V) \otimes K^{h}(W) \rightarrow K^{h}(V \oplus W)^{\otimes 2} \stackrel{m}{\rightarrow} K^{h}(V \oplus W) .
$$

Finally, we observe that when $V$ is equipped with weights, $K^{h}(V)$ is canonically made into a wdg-ZZ-algebra.

Assume now that $V$ is concentrated in even degrees. We can adapt the definition of the dual Koszul algebra by exchanging the roles of exterior and divided powers. This yields the dual De Rham algebra $\Omega^{h}(V)$. 
Definition 11.2 (Dual De Rham algebra). Let $V$ be a positively graded $\mathbb{Z}$ - free module, and let $h$ be an integer. The dual De Rham algebra $\Omega^{h}(V)$ is the cdg-Z्Z-algebra which equals $\Gamma(V) \otimes \Lambda(V[1])$ as a commutative graded algebra and whose differential equals the composite

$$
\begin{aligned}
\Gamma^{n}(V) \otimes \Lambda^{k}(V[1]) & \stackrel{\operatorname{Id} \otimes \Delta}{\longrightarrow} \Gamma^{n}(V) \otimes V[1] \otimes \Lambda^{k-1}(V[1]) \\
& \stackrel{h \mathrm{Id}}{\longrightarrow} \Gamma^{n}(V) \otimes V \otimes \Lambda^{k-1}(V[1]) \stackrel{m \otimes \mathrm{Id}}{\longrightarrow} \Gamma^{n+1}(V) \otimes \Lambda^{k-1}(V[1]) .
\end{aligned}
$$

If $h=1, \Omega^{h}(V)$ is the graded dual of the usual De Rham algebra, and if $V$ is a copy of $\mathbb{Z}$ in even degree, it is an elementary complex of type (II) from [Car, Exp 11]. There are isomorphisms of dg-Z-Z-algebras $\Omega^{h}(V) \otimes \Omega^{h}(W) \simeq$ $\Omega^{h}(V \oplus W)$, and when $V$ is equipped with weights, $\Omega^{h}(V)$ becomes a wdg$\mathbb{Z}$-algebra.

11.2. Cartan's result. We fix a free $\mathbb{Z}$-module $V=\mathbb{Z}^{m}$. and a positive integer $n$. We are going to present Cartan's computation of the homology of EML spaces from [Car, Exp. 11, Theoreme 1]. Recall from definition 10.6 the $p$-admissible words of height $n$ attached to a prime $p$ (here we also use definition 10.6 when $p=2$ ). The word $\sigma^{n}$ is $p$-admissible of height $n$, and the other $p$-admissible words of height $n$ can be grouped in a unique way into pairs of the form $\left(\sigma^{k+1} \gamma_{p} \alpha, \sigma^{k} \phi_{p} \alpha\right)$, where $\alpha$ is denotes a word and $k \geq 0$. In such a pair, the degrees of the two words differ by one: $\operatorname{deg}\left(\sigma^{k} \phi_{p} \alpha\right)=\operatorname{deg}\left(\sigma^{k+1} \gamma_{p} \alpha\right)+1$.

Definition 11.3. We call p-pair of height $n$ a pair of $p$-admissible words of height $n$ of the form $\underline{\pi}=\left(\sigma^{k+1} \gamma_{p} \alpha, \sigma^{k} \phi_{p} \alpha\right)$ (where $\alpha$ is a word, and $k \geq 0$ ). The degree of the pair $\underline{\pi}$ is the lowest degree of the words of the pair, that is $\operatorname{deg}(\underline{\pi})=\operatorname{deg}\left(\sigma^{k+1} \gamma_{p} \alpha\right)$.

Example 11.4. If $n=3$, the $p$-pairs of height $n$ are the pairs

$$
\underline{\pi}_{k}=\left(\sigma \gamma_{p}^{k+1} \sigma^{2}, \phi_{p} \gamma_{p}^{k} \sigma^{2}\right), \quad \text { for } k \geq 0,
$$

and $\operatorname{deg}\left(\underline{\pi}_{k}\right)=2 p^{k+1}+1$. If $n=4$ the $p$-pairs of height $n$ are the pairs

$$
\begin{array}{ll}
\underline{\pi}_{\ell}=\left(\sigma^{2} \gamma_{p}^{\ell+1} \sigma^{2}, \sigma \phi_{p} \gamma_{p}^{\ell} \sigma^{2}\right), & \text { for } \ell \geq 0, \\
\underline{\pi}_{k, \ell}=\left(\sigma \gamma_{p}^{k+1} \phi_{p} \gamma_{p}^{\ell} \sigma^{2}, \phi_{p} \gamma_{p}^{k} \phi_{p} \gamma_{p}^{\ell} \sigma^{2}\right), & \text { for } k \geq 0, \ell \geq 0,
\end{array}
$$

and their degrees are $\operatorname{deg}\left(\underline{\pi}_{\ell}\right)=2 p^{\ell+1}+2, \operatorname{deg}\left(\underline{\pi}_{k, \ell}\right)=2 p^{\ell+k+2}+2 p^{k+1}+1$.

Definition 11.5. We denote by $X_{p}^{[n]}$ the cdg-Z-Zalgebra defined by:

$$
X_{p}^{[n]}=K^{p}\left(\bigoplus_{\underline{\pi}} V[\operatorname{deg}(\underline{\pi})]\right) \otimes \Omega^{p}\left(\bigoplus_{\underline{\pi}^{\prime}} V\left[\operatorname{deg}\left(\underline{\pi}^{\prime}\right)\right]\right),
$$

where the first direct sum is taken over all the $p$-pairs $\underline{\pi}$ of height $n$ and odd degree, and the second one is taken over all the $p$-pairs $\underline{\pi}^{\prime}$ of height $n$ and even degree. We also denote by $X_{0}^{[n]}$ the cdg-k-algebra which equals $\Lambda(V[n])$ if $n$ is odd and $\Gamma(V[n])$ if $n$ is even, with trivial differential.

Let us denote by ${ }_{p} M$ the $p$-primary part of a $\mathbb{Z}$-module $M$ :

$$
{ }_{p} M=\left\{m \in M ; \exists k p^{k} m=0\right\} .
$$


The homology of $X_{p}^{[n]}$ equals $\mathbb{Z}$ in degree zero, so its $p$-primary part ${ }_{p} H\left(X_{p}^{[n]}\right)$ is a graded subalgebra of $H\left(X_{p}^{[n]}\right)$ without unit (it is concentrated in positive degrees). We make it into a unital $\mathbb{Z}$-algebra $\widehat{{ }_{p} H}\left(X_{p}^{[n]}\right)$ in the canonical way:

$$
\widehat{{ }_{p} H_{0}}\left(X_{p}^{[n]}\right)=\mathbb{Z}, \quad \widehat{{ }_{p} H_{i}}\left(X_{p}^{[n]}\right)={ }_{p} H_{i}\left(X_{p}^{[n]}\right) \text { for } i>0 .
$$

In view of corollary 8.3, Cartan's computation of the integral homology of the EML-spaces $K\left(\mathbb{Z}^{m}, n+2\right)$ can be formulated in the following way.

Theorem 11.6 ([Car, Exp. 11, Theoreme 1]). Let $n$ be a positive integer, let $V=\mathbb{Z}^{m}$ be a free abelian group. The homology of $\bar{B}^{n} \Gamma(V[2])$ is isomorphic as a graded algebra, to the tensor product

$$
X_{0}^{[n+2]} \otimes \bigotimes_{p \text { prime }} \widehat{p^{H}}\left(X_{p}^{[n+2]}\right) .
$$

11.3. Integral torsion of Exts. To be able to identify $\mathbb{E}^{i}\left(S^{d}, \Lambda^{d}\right)(V)$ or $\mathbb{E}^{i}\left(S^{d}, \Gamma^{d}\right)(V)$ explicitly as a direct summand of the homology of $\bar{B}^{n} \Gamma(V[2])$, we have to describe the later as a graded algebra with weights. However, some interesting information can already be extracted from theorem 11.6 without describing the weights, in particular for the case $n=1$.

Indeed, observe in example 11.4 that all the $p$-pairs of height 3 have odd degrees. So for all prime $p, X_{p}^{[3]}$ is just the dual Koszul algebra

$$
X_{p}^{[3]}=K^{p}\left(\bigoplus_{k \geq 0} V\left[2 p^{k+1}+1\right]\right) .
$$

Lemma 11.7. $H_{i}\left(X_{p}^{[3]}\right)$ is a $\mathbb{F}_{p}$-vector space if $i>0$ and equals $\mathbb{Z}$ if $i=0$.

Proof. If $W$ is a graded free $\mathbb{Z}$-module in positive odd degrees, the dual Koszul algebra $K^{1}(W)$ satisfies $H_{i}\left(K^{1}(W)\right)=\mathbb{Z}$ if $i=0$ and zero otherwise (use the exponential formula and the Künneth morphism to reduce to the case when $W$ has rank one, then the result is easy). Since the differential $\partial$ of $K^{p}(W)$ is $p$ times the differential $\delta$ of $K^{1}(W)$, we have $H_{0}\left(K^{p}(W)\right)=\mathbb{Z}$, and if $i>0$ :

$$
H_{i}\left(K^{p}(W)\right)=\operatorname{Ker} \partial / \operatorname{Im} \partial=\operatorname{Ker} \delta / p(\operatorname{Im} \delta)=\operatorname{Im} \delta / p(\operatorname{Im} \delta) \simeq \operatorname{Im} \delta \otimes_{\mathbb{Z}} \mathbb{F}_{p} .
$$

This proves the result.

Lemma11.7implies that the $p$-primary part of the homology of $\bar{B} \Gamma(V[2])$, hence of $\mathbb{E}(S, \Lambda)(V)$, is a $\mathbb{F}_{p}$-vector space. This information is precious because it implies that $\mathbb{E}(S, \Lambda)(V)$ can be retrieved from the analogous extensions over $\mathbb{F}_{p}$ by the universal coefficient theorem.

Theorem 11.8. Let $V=\mathbb{Z}^{m}$. Then $\mathbb{E}^{0}\left(S^{d}, \Lambda^{d}\right)(V) \simeq \Gamma^{d}(V)$, and for $i$ positive, the p-primary part of $\mathbb{E}^{i}\left(S^{d}, \Lambda^{d}\right)(V)$ is a finite dimensional $\mathbb{F}_{p^{-}}$ vector space. Moreover let $P(t)$, resp. $Q(t)$, be the Poincaré series of the graded $\mathbb{F}_{p}$-vector space $\mathbb{E}\left(S^{d}, \Lambda^{d}\right)(V) \otimes_{\mathbb{Z}} \mathbb{F}_{p}$, resp. $\mathbb{E}\left(S_{\mathbb{F}_{p}}^{d}, \Lambda_{\mathbb{F}_{p}}^{d}\right)(V / p V)$. Then we have $(1+t) P(t)=t Q(t)+P(0)$. 
Proof. The computation of $\mathbb{E}^{0}\left(S^{d}, \Lambda^{d}\right)(V)$ was made in lemma 4.6. It is well known that $\mathbb{E}^{i}\left(S^{d}, \Lambda^{d}\right)(V)$ is a finite abelian group for $i$ positive. This is actually true for extensions between arbitrary strict polynomial functors $F, G$ with finitely generated values, but in our case it can be directly proved as follows. First, $\mathbb{E}^{i}\left(S^{d}, \Lambda^{d}\right)(V)$ is a finitely generated abelian group. Indeed, it is the $d-i$-th homology group of the complex $\mathbb{H}\left(S^{d}, I_{\bullet}, d\right)(V)$ from section 7.2 , which is a complex of free finitely generated $\mathbb{Z}$-modules. Then by base change $\left[\mathrm{SFB}\right.$, prop 2.6] and the universal coefficient theorem, $\mathbb{E}^{i}\left(S^{d}, \Lambda^{d}\right)(V) \otimes_{\mathbb{Z}} \mathbb{Q}$ is isomorphic to $\mathbb{E}^{i}\left(S_{\mathbb{Q}}^{d}, \Lambda_{\mathbb{Q}}^{d}\right)\left(V \otimes_{\mathbb{Z}} \mathbb{Q}\right)$ which is zero because the category $\mathcal{P}_{\mathbb{Q}}$ is semi-simple. Thus $\mathbb{E}^{i}\left(S^{d}, \Lambda^{d}\right)(V)$ is a finite abelian group. Now lemma 11.7 and theorem 11.6 show that the $p$-primary part of $\bar{B} \Gamma(V[2])$, hence of

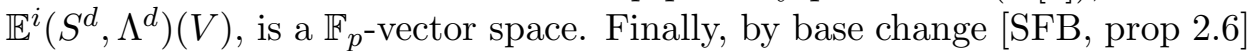
and the universal coefficient theorem, there is for all $i$ an isomorphism of $\mathbb{F}_{p}$-vector spaces:

$$
\mathbb{E}^{i}\left(S^{d}, \Lambda^{d}\right)(V) \otimes_{\mathbb{Z}} \mathbb{F}_{p} \oplus \mathbb{E}^{i+1}\left(S^{d}, \Lambda^{d}\right)(V) \otimes_{\mathbb{Z}} \mathbb{F}_{p} \simeq \mathbb{E}^{i}\left(S_{\mathbb{F}_{p}}^{d}, \Lambda_{\mathbb{F}_{p}}^{d}\right)(V / p V) .
$$

The assertion on the Poincaré series follows.

The Poincaré series $Q(t)$ are easy to determine from theorems 10.16 and 10.17, and they were first determined in $\mathrm{A}]$ for $V=\mathbb{Z}$. Since we know $P(0)=\operatorname{dim}_{\mathbb{F}_{p}}\left(\Gamma^{d}(V) \otimes \mathbb{F}_{p}\right)$, the Poincaré series $P(t)$ are easily computed from the equation $(1+t) P(t)=t Q(t)+P(0)$ of theorem 11.8. To illustrate this, we give the values of $\operatorname{Ext}_{\mathcal{P}_{\mathbb{Z}}}^{i}\left(S^{n}, \Lambda^{n}\right)$ in low degrees (for $i=1$ they were first computed in [A, section 4]).

Example 11.9. We have the following Ext-computations:

$$
\begin{aligned}
& \operatorname{Ext}_{\mathcal{P}_{\mathbb{Z}}}^{1}\left(S^{n}, \Lambda^{n}\right)=\mathbb{Z} / 2 \mathbb{Z} \text { if } n \geq 2, \text { and zero if } n \leq 1, \\
& \operatorname{Ext}_{\mathcal{P}_{\mathbb{Z}}}^{2}\left(S^{n}, \Lambda^{n}\right)=\mathbb{Z} / 3 \mathbb{Z} \text { if } n=3,4 \text { and zero otherwise, } \\
& \operatorname{Ext}_{\mathcal{P}_{\mathbb{Z}}}^{3}\left(S^{n}, \Lambda^{n}\right)=\left\{\begin{array}{cl}
0 & \text { if } n \leq 3, \\
\mathbb{Z} / 2 \mathbb{Z} \oplus \mathbb{Z} / 3 \mathbb{Z} & \text { if } n=6,7, \\
\mathbb{Z} / 2 \mathbb{Z} & \text { otherwise. }
\end{array}\right.
\end{aligned}
$$

In the case $n=2, X_{p}^{[4]}$ is a tensor product of a dual Koszul algebra and a dual De Rham algebra. The latter brings $p^{r}$-torsion for all values of $r \geq 0$ in the homology of $X_{p}^{[4]}$. So in contrast to theorem 11.8 we have the following result.

Proposition 11.10. Let $V=\mathbb{Z}^{m}$. For all prime $p$ and all $r \geq 0$, there exists positive integers $i, d$ such that the abelian group $\mathbb{E}^{i}\left(S^{d}, \Gamma^{d}\right)(V)$ has an element of $p^{r}$-torsion.

11.4. Computation of weights. The $\mathcal{P}_{\mathbb{Z}}$-dg-algebra $\bar{B}^{n}(\Gamma[2])$ becomes a wdg-Z्Z-algebra after evaluation on $V=\mathbb{Z}^{m}$, so the homology of $\bar{B}^{n} \Gamma(V[2])$ is equipped with weights. We are now going to supplement Cartan's result by describing these weights.

Definition 11.11. Let $\underline{\pi}$ be a $p$-pair. The weight of $\underline{\pi}$ is the integer $w(\underline{\pi})$ defined by $w(\underline{\pi})=p^{t_{\underline{\pi}}}$, where $t_{\underline{\pi}}$ is the number of letters which equal $\phi_{p}$ or $\gamma_{p}$ in one of the two words of $\underline{\pi}$ (compare definition 10.13). 
Example 11.12. We keep the notations of example 11.4, For the p-pairs of height $n=3$, we have $w\left(\underline{\pi}_{k}\right)=p^{k+1}$. For the $p$-pairs of height 4 we have: $w\left(\underline{\pi}_{\ell}\right)=p^{\ell+1}$ and $w\left(\underline{\pi}_{k, \ell}\right)=p^{k+\ell+2}$.

Theorem 11.13. Let $n$ be a nonnegative integer, let $V=\mathbb{Z}^{m}$ be a free abelian group. There is an isomorphism of graded algebras with weights

$$
X_{0}^{[n+2]} \otimes \bigotimes_{p \text { prime }} \widehat{{ }_{p}} \widehat{H}\left(X_{p}^{[n+2]}\right) \simeq H\left(\bar{B}^{n}(\Gamma(V[2]))\right)
$$

On the left hand side, $X_{p}^{[n+2]}$ denotes the $w d g$-ZZ-algebra defined by:

$$
X_{p}^{[n+2]}=K^{p}\left(\bigoplus_{\underline{\pi}} V_{w(\underline{\pi})}[\operatorname{deg}(\underline{\pi})]\right) \otimes \Omega^{p}\left(\bigoplus_{\underline{\pi^{\prime}}} V_{w\left(\underline{\pi}^{\prime}\right)}\left[\operatorname{deg}\left(\underline{\pi}^{\prime}\right)\right]\right),
$$

where $V_{k}[i]$ denotes a copy of $V$ having weight $k$ and degree $i$, and where the first direct sum is taken over a the p-pairs $\underline{\pi}$ of height $n+2$ and odd degree and the second direct sum is taken over the p-pairs $\underline{\pi}^{\prime}$ of height $n+2$ and even degree. Moreover, $X_{0}^{[n+2]}$ equals $\Lambda\left(V_{1}[n+2]\right)$ if $n$ is odd, and $\Gamma\left(V_{1}[n+2]\right)$ if $n$ is even.

Before we prove theorem 11.13, we recall the universal property of dual Koszul and De Rham algebras. If $W$ is a graded $\mathbb{Z}$-module we denote by $W_{\text {odd }}$, resp. $W_{\text {even }}$, its summand of odd, resp. even, degree.

Lemma 11.14 (see [Car, Exp. 1, section 2]). Let $W$ be a positively graded $\mathbb{Z}$-free module, let $h$ be a integer, and let $C^{h}(W)$ be the complex $W[1] \stackrel{h \mathrm{Id}}{\longrightarrow} W$. This complex is a direct summand of $K^{h}\left(W_{\text {odd }}\right) \otimes \Omega^{h}\left(W_{\text {even }}\right)$. Assume that $A$ is a cdg-Z्Z-algebra, equipped with a system of divided powers, and free as a $\mathbb{Z}$ module. For all morphism of complexes $f: C^{h}(W) \rightarrow A$ there exists a unique morphism of cdg-Z-Z-algebras $\bar{f}$ such that the following diagram commutes:

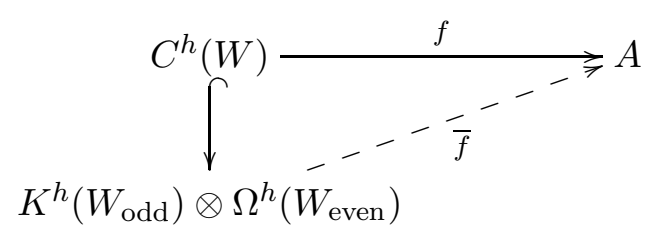

If $W$ is equipped with weights and $A$ is a cwdg-Z्Z-algebra, then $\bar{f}$ preserves the weights if and only if $f$ does.

Proof. The morphism of algebra $\bar{f}$ is obtained by the universal property of the graded algebra with divided powers $K^{h}\left(W_{\text {odd }}\right) \otimes \Omega^{h}\left(W_{\text {even }}\right)=U(W[1] \oplus$ $W$ ) (see theorem [10.2). In particular $\bar{f}$ preserves the weights if and only if $f$ does. So one has only to check that $\bar{f}$ commutes with the differentials. Using that $d\left(\gamma^{k}(x)\right)=d x \cdot \gamma^{k-1} x$ in $K^{h}\left(W_{\text {odd }}\right) \otimes \Omega^{h}\left(W_{\text {even }}\right)=U(W[1] \oplus W)$ and $A$, one reduces the proof that $\bar{f}$ commutes with differentials to the proof that the restriction of $\bar{f}$ to the generators of $K^{h}\left(W_{\text {odd }}\right) \otimes \Omega^{h}\left(W_{\text {even }}\right)=$ $U(W[1] \oplus W)$ commutes with the differential. But this restriction is nothing but $f$. Whence the result. 
Proof of theorem 11.13. Denote by $H(V, n+2, \mathbb{k})$ the singular homology of the EML space $K(V, n+2)$ with coefficients in $\mathbb{k}$. Let $A$ be a differential graded $\mathbb{Z}$-algebra such that

(i) $A$ is graded commutative, $\mathbb{Z}$-free, equipped with divided powers,

(ii) The homology of $A$ is isomorphic to $H(V, n+2, \mathbb{Z})$,

(iii) For all prime $p$, the homology of the $\mathbb{F}_{p}$-differential graded algebra $A \otimes_{\mathbb{Z}} \mathbb{F}_{p}$ is isomorphic to $H\left(V, n+2, \mathbb{F}_{p}\right)$.

To prove theorem 11.6. Cartan builds morphisms of dg-algebras

$$
f_{p}: X_{p}^{[n+2]} \rightarrow A,
$$

(for $p$ zero or prime) which induce after taking homology the isomorphism

$$
X_{0}^{[n+2]} \otimes \bigotimes_{p \text { prime } p} \widehat{H}\left(X_{p}^{[n+2]}\right) \simeq H(V, n+2, \mathbb{Z}) .
$$

In Cartan's proof, the algebra $A$ is $\bar{B}^{n+2}(\mathbb{Z} V)$, but any other algebra $A$ satisfying conditions $(i),(i i)$ and $(i i i)$ works as well in his argument, for example we can take $A=\bar{B}^{n}(\Gamma(V[2]))$.

So, to prove theorem 11.13, it suffices to prove that the morphisms $f_{p}$ preserve weights when $A=\bar{B}^{n} \Gamma(V[2])$ and when the weights on $X_{p}^{[n+2]}$ are as indicated in the statement of theorem 11.13 .

Case of $f_{p}, p$ prime. By the universal property of Dual Koszul and De Rham algebras from lemma 11.14, the construction of $f_{p}$ (preserving weights) reduces to the construction for each $p$-pair $\underline{\pi}$ of a morphism of complexes (preserving weights)

$$
f_{p}: C^{p}\left(V_{w(\underline{\pi})}[\operatorname{deg}(\underline{\pi})]\right) \rightarrow A
$$

Fix a pair $\underline{\pi}=\left(\sigma^{k+1} \gamma_{p} \alpha, \sigma^{k} \phi_{p} \alpha\right)$ and a basis $\left(v_{i}\right)$ of $V[\operatorname{deg}(\underline{\pi})]$. Denote by $\left(w_{i}\right)$ the same basis, considered as a basis of $V[\operatorname{deg}(\underline{\pi})+1]$. So the complex $C^{p}\left(V_{w(\underline{\pi})}[\operatorname{deg}(\underline{\pi})]\right)$ equals

$$
V[\operatorname{deg}(\underline{\pi})+1] \stackrel{d}{\rightarrow} V[\operatorname{deg}(\underline{\pi})],
$$

with $d\left(w_{i}\right)=v_{i}$. Let $\overline{v_{i}}$ be the reduction modulo $p$ of $v_{i}$, considered as an element of $V \otimes_{\mathbb{Z}} \mathbb{F}_{p}\left[\operatorname{deg}\left(\sigma^{k} \phi_{p} \alpha\right)\right]$ which is the direct summand $H\left(V, n+2, \mathbb{F}_{p}\right)$ indexed by the $p$-admissible word $\sigma^{k} \phi_{p} \alpha$, cf. theorem 10.14. This summand is also well defined for $p=2$ by remark 10.15. The morphism of complexes $f_{p}$ is defined by Cartan as follows.

(a) The canonical map $A \rightarrow A \otimes_{\mathbb{Z}} \mathbb{F}_{p}$ is surjective so we can find an element $f_{p}\left(w_{i}\right) \in A$ whose reduction modulo $p$ is a cycle representing the homology class $\overline{v_{i}} \in H\left(V, n+2, \mathbb{F}_{p}\right)$.

(b) The reduction mod $p$ of $f_{p}\left(w_{i}\right)$ is a cycle of $A \otimes_{\mathbb{Z}} \mathbb{F}_{p}$, so $d\left(f_{p}\left(w_{i}\right)\right)$ is divisible by $p$. We define $f_{p}\left(v_{i}\right)$ by the equality $p f_{p}\left(v_{i}\right)=d\left(f_{p}\left(w_{i}\right)\right)$.

Take $A=\bar{B}^{n} \Gamma(V[2])$. The canonical map $A \rightarrow A \otimes_{\mathbb{Z}} \mathbb{F}_{p}$ preserves weights, so the weight of $f_{p}\left(w_{i}\right)$ is the same as the weight of $\bar{v}_{i}$, which equals $w(\underline{\pi})$ by theorem 10.14. Thus, the map $f_{p}$ preserves weights.

Case of $f_{0}$. The morphism $f_{0}: X_{0}^{[n+2]} \rightarrow \bar{B}^{n}(\Gamma(V[2]))$ is induced by the morphism $V_{1}[n+2] \stackrel{\equiv}{\rightarrow} \bar{B}^{n}(\Gamma(V[2]))_{n+2}$ and the universal property of $X_{0}^{[n+2]}=U\left(V_{1}[n+2]\right)$. So $f_{0}$ preserves weights (it is actually a morphism of $\mathcal{P}_{\mathbb{Z}}$-dg-algebras). 
Theorem 11.13 yields an algorithm to compute the homology of $\bar{B}^{n} \Gamma(V[2])$. Indeed, the homology of the dual Koszul and De Rham algebras on a single generator can be computed by direct inspection.

(i) The homology of $K^{h}(\mathbb{Z}[2 i-1])$ is $\mathbb{Z}$ in degree 0 (and weight zero), $\mathbb{Z} / h \mathbb{Z}$ in degrees $d 2 i-1$ (and weight $d$ ) for $d \geq 0$, and zero elsewhere.

(ii) The homology of $\Omega^{h}(\mathbb{Z}[2 i])$ is $\mathbb{Z}$ in degree 0 (and weight zero), $\mathbb{Z} / d h \mathbb{Z}$ in degrees $d 2 i$ (and weight $d$ ) for $d \geq 0$, and zero elsewhere.

With the help of the exponential isomorphisms

$K^{h}(V \oplus W) \simeq K^{h}(V) \otimes K^{h}(W), \quad$ and $\quad \Omega^{h}(V \oplus W) \simeq \Omega^{h}(V) \otimes \Omega^{h}(W)$,

and iterative uses of the Künneth formula, one recovers the homology of the wdg-Z $\mathbb{Z}$-algebras $X_{p}^{[n+2]}$. Theses algebras have an infinite number of generators, but only a (relatively small) finite number of generators play a role in the computation of a summand of the homology with given weight or degree. Let us give the computation of the homogeneous part of weight 4 of the homology of $\bar{B}^{n} \Gamma(\mathbb{Z}[2])$ for $n=1$ and $n=2$.

Example 11.15. The homogeneous part of weight 4 of the homology of $\bar{B} \Gamma(\mathbb{Z}[2])$ is given by the following table (it is zero outside the table).

\begin{tabular}{c|ccc} 
degree & 9 & 10 & 11 \\
\hline homology & $\mathbb{Z} / 2 \mathbb{Z}$ & $\mathbb{Z} / 3 \mathbb{Z}$ & $\mathbb{Z} / 2 \mathbb{Z}$
\end{tabular}

The homogeneous part of weight 4 of the homology of $\bar{B}^{2} \Gamma(\mathbb{Z}[2])$ is given by the following table (it is zero outside the table).

\begin{tabular}{c|ccccccc} 
degree & 10 & 11 & 12 & 13 & 14 & 15 & 16 \\
\hline homology & $\mathbb{Z} / 2 \mathbb{Z}$ & 0 & $\mathbb{Z} / 12 \mathbb{Z}$ & $\mathbb{Z} / 2 \mathbb{Z}$ & $\mathbb{Z} / 2 \mathbb{Z}$ & 0 & $\mathbb{Z}$
\end{tabular}

11.5. The algebra of Koszul kernels. When $n=1$, theorem 11.13 has a nicer formulation in terms of the algebra of Koszul kernels.

If $W$ is a graded $\mathbb{F}_{p}$-vector space concentrated in positive odd degrees, we denote by $K_{\mathbb{F}_{p}}(W)$ the dual Koszul algebra over $W$, that is, the graded $\mathbb{F}_{p}$-algebra $\Gamma_{\mathbb{F}_{p}}(W[1]) \otimes_{\mathbb{F}_{p}} \Lambda_{\mathbb{F}_{p}}(W)$ (the index ${ }^{~} \mathbb{F}_{p}$ ' is put here to emphasize that we work in the realm of $\mathbb{F}_{p}$-vector spaces, in particular $\Gamma_{\mathbb{F}_{p}}(W[1])$ and $\Lambda_{\mathbb{F}_{p}}(W)$ equal $\mathbb{F}_{p}$ in degree zero) equipped with the Koszul differential, defined as the composite

$$
\begin{aligned}
& \Gamma_{\mathbb{F}_{p}}^{n}(W[1]) \otimes_{\mathbb{F}_{p}} \Lambda_{\mathbb{F}_{p}}^{k}(W) \stackrel{\Delta \otimes \mathbb{I d}}{\longrightarrow} \Gamma_{\mathbb{F}_{p}}^{n-1}(W[1]) \otimes_{\mathbb{F}_{p}} W[1] \otimes_{\mathbb{F}_{p}} \Lambda_{\mathbb{F}_{p}}^{k}(W) \\
& \quad \stackrel{\mathrm{Id}}{\longrightarrow} \Gamma_{\mathbb{F}_{p}}^{n-1}(W[1]) \otimes_{\mathbb{F}_{p}} W \otimes_{\mathbb{F}_{p}} \Lambda_{\mathbb{F}_{p}}^{k}(W) \stackrel{\text { Id } \otimes m}{\longrightarrow} \Gamma_{\mathbb{F}_{p}}^{n-1}(W[1]) \otimes_{\mathbb{F}_{p}} \Lambda_{\mathbb{F}_{p}}^{k+1}(W) .
\end{aligned}
$$

The dual Koszul algebra is a cdg- $\mathbb{F}_{p}$-algebra, it has weights if $W$ is equipped with weights, and it is a $\mathcal{P}_{\mathbb{F}_{p}}$-graded algebra if $W$ is a graded strict polynomial functor.

Definition 11.16. Let $W$ be a graded $\mathbb{F}_{p}$-vector space concentrated in positive odd degrees. The algebra of Koszul kernels $\mathbb{K}_{\mathbb{F}_{p}}(W)$ is the commutative graded $\mathbb{Z}$-algebra defined by: $\mathbb{K}_{\mathbb{F}_{p}}(W)_{0}=\mathbb{Z}$ and in positive degrees $\mathbb{K}_{\mathbb{F}_{p}}(W)$ equals the cycles of positive degree of the dual Koszul algebra $K_{\mathbb{F}_{p}}(W)$. 
Lemma 11.17. Let $V$ be a graded $\mathbb{Z}$-module with weights, concentrated in odd degrees and $\mathbb{Z}$-free of finite rank in each degree. Put the weights on $V / p V$ such that the epimorphism $V \rightarrow V / p V$ preserves weights. There is an isomorphism of graded $\mathbb{Z}$-algebras with weights:

$$
H\left(K^{p}(V)\right) \simeq \mathbb{K}_{\mathbb{F}_{p}}(V / p V) .
$$

Proof. We have already proved in lemma 11.7 that $H_{0}\left(K^{p}(V)\right) \simeq \mathbb{Z}$ and that for positive $i, H_{i}\left(K^{p}(V)\right)$ is isomorphic to the image of the differential of $K^{1}(V)$ tensored by $\mathbb{F}_{p}$. But $K^{1}(V) \otimes \mathbb{F}_{p} \simeq K_{\mathbb{F}_{p}}(V / p V)$ so the latter equals $\operatorname{Im}(d)$ where $d$ is the differential of $K_{\mathbb{F}_{p}}(V / p V)$. Finally, since $K_{\mathbb{F}_{p}}(V / p V)$ is exact in positive degrees (use the exponential formula and the Künneth isomorphism to reduce to the case when $V$ has rank one), $\operatorname{Im}(d)=\mathbb{K}_{\mathbb{F}_{p}}(V / p V)$.

Using lemma 11.17, the case $n=1$ of theorem 11.13 can be reformulated in the following way.

Theorem 11.18. Let $V[2]$ be a free $\mathbb{Z}$-module of finite rank concentrated in degree 2 and weight one. The homology of $\bar{B}(\Gamma(V[2]))$ is isomorphic to the graded algebra with weights (where $(V / p V)^{(r)}[k]$ denotes a copy of $V$ with degree $k$ and weight $p^{r}$ ):

$$
\Lambda(V[3]) \otimes \bigotimes_{p \text { prime }} \mathbb{K}_{\mathbb{F}_{p}}\left(\bigoplus_{k \geq 0}(V / p V)^{(k+1)}\left[2 p^{k+1}+1\right]\right) .
$$

Observe that the graded algebra with weights appearing in theorem 11.18 is actually a graded $\mathcal{P}_{\mathbb{Z}}$-algebra. We do not claim in theorem 11.18 that the isomorphism is an isomorphism of graded $\mathcal{P}_{\mathbb{Z}}$-algebras, but however we conjecture that this is the case, at least for the $p$-primary part when $p$ is odd. The proof of this (and further developments) is a work in progress with L. Breen and R. Mikhailov [BMT].

11.6. Ext-computations over the integers. By theorem 7.5 the graded algebras with weights $\mathbb{E}(S, \Lambda)\left(\mathbb{Z}^{m}\right)$ and $\mathbb{E}(S, \Gamma)\left(\mathbb{Z}^{m}\right)$ are simply obtained from $\bar{B}^{n}\left(\Gamma\left(\mathbb{Z}^{m}[2]\right)\right)$ by regrading. For instance, we obtain the following Extcomputation from example 11.15 .

Example 11.19. The groups $\operatorname{Ext}_{\mathcal{P}_{\mathbb{Z}}}^{i}\left(S^{4}, \Lambda^{4}\right)$ and $\operatorname{Ext}_{\mathcal{P}_{\mathbb{Z}}}^{i}\left(S^{4}, \Gamma^{4}\right)$ are given by the following tables (they are zero outside the table).

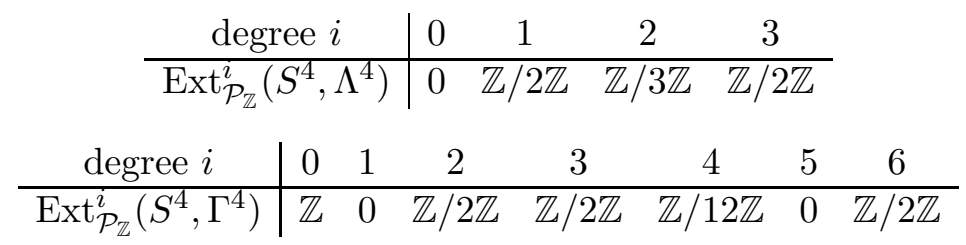

We can also give general formulas by regrading theorems 11.13 and 11.18 . If $W$ is a graded $\mathbb{F}_{p}$-module with weights concentrated in even degrees, we let $\widetilde{\mathbb{K}}_{\mathbb{F}_{p}}(W)$ be the wg-Z्Z-algebra which equals $\mathbb{Z}$ in degree zero and which equals the subalgebra of cycles of the wdg- $\mathbb{Z}$-algebra ${ }^{t} \Gamma_{\mathbb{F}_{p}}(W[1]) \otimes 1 \Lambda_{\mathbb{F}_{p}}(W)$ (equipped with the Koszul differential) in positive degrees. So $\widetilde{\mathbb{K}}_{\mathbb{F}_{p}}(W)$ is 
equal to the algebra of Koszul kernels $\mathbb{K}_{\mathbb{F}_{p}}(W)$ if $p=2$, and it differs from $\mathbb{K}_{\mathbb{F}_{p}}(W)$ by signs in the multiplication if $p$ is odd. Moreover:

$$
\widetilde{\mathbb{K}}_{\mathbb{F}_{p}}\left(\mathcal{R}_{3} V\right)=\mathcal{R}_{3}\left(\mathbb{K}_{\mathbb{F}_{p}}(V)\right)
$$

when $V$ is a graded $\mathbb{F}_{p}$-module with weights concentrated in odd degrees and odd weights, or when $p=2$. Thus, theorem 11.18 and theorem 7.5 yield the following computation of $\mathbb{E}(S, \Lambda)\left(\mathbb{Z}^{m}\right)$.

Theorem 11.20. Let $\mathbb{Z}^{m}$ be a free abelian group. There is an isomorphism of graded $\mathbb{Z}$-algebras with weights

$\mathbb{E}(S, \Lambda)\left(\mathbb{Z}^{m}\right) \simeq \Lambda\left(\mathbb{Z}^{m}\langle 0\rangle\right) \otimes^{1} \bigotimes_{p \text { prime }}^{1} \widetilde{\mathbb{K}}_{\mathbb{F}_{p}}\left(\bigoplus_{k \geq 0}\left(\mathbb{Z}^{m} / p \mathbb{Z}^{m}\right)^{(k+1)}\left\langle p^{k+1}-1\right\rangle\right)$.

On the right hand side, $\mathbb{Z}^{m}\langle 0\rangle$ is a copy of $\mathbb{Z}^{m}$ having degree zero and weight 1 , and $\left(\mathbb{Z}^{m} / p \mathbb{Z}^{m}\right)^{(k)}\langle i\rangle$ denotes a copy of $\mathbb{Z}^{m} / p \mathbb{Z}^{m}$ having cohomological degree $i$ and weight $p^{k}$.

Using theorems 11.18 and theorem 7.5, we also obtain a computation of the graded $\mathbb{Z}$-algebras with weights $\mathbb{E}(S, \Gamma)\left(\mathbb{Z}^{m}\right)$.

Theorem 11.21. Let $\mathbb{Z}^{m}$ be a free abelian group. There is an isomorphism of graded $\mathbb{Z}$-algebras with weights

$$
\mathbb{E}(S, \Gamma)\left(\mathbb{Z}^{m}\right) \simeq \Gamma\left(\mathbb{Z}^{m}\langle 0\rangle\right) \otimes \bigotimes_{p \text { prime }} \widehat{{ }_{p} H}\left(\widetilde{X}_{p}\right) .
$$

On the right hand side, $\widetilde{X}_{p}$ is the $w d g-\mathbb{Z}$-algebra defined as the tensor product: $K^{p}\left(\bigoplus_{k, \ell \geq 0} \mathbb{Z}_{p^{k+\ell+2}}^{m}\left\langle 2 p^{k+\ell+2}-2 p^{k+1}-1\right\rangle\right) \otimes \Omega^{p}\left(\bigoplus_{\ell \geq 0} \mathbb{Z}^{m}{ }^{\ell+1}\left\langle 2 p^{k+1}-2\right\rangle\right)$, where $\mathbb{Z}^{m}{ }_{k}\langle i\rangle$ denotes a copy of $\mathbb{Z}^{m}$ having weight $k$ and cohomological degree $i$ (or equivalently homological degree $-i$ ).

\section{Part 4. Frobenius twists}

In this part, $\mathbb{k}$ is a field of prime characteristic $p$. We compute the $\mathcal{P}_{\mathbb{k}^{-}}$ graded algebras $\mathbb{E}\left(X^{(r)}, Y^{(s)}\right)$, when $X$ and $Y$ are classical exponential functors and $r, s$ are nonnegative integers. Our method offers a unified treatment of all the cases. We proceed in several steps.

In section 12, we show that the $\mathcal{P}_{\mathbb{k}^{k}}$-graded algebras of the form $\mathbb{E}\left(X^{(t)}, Y\right)$ are equal, up to a regrading, to the algebras $\mathbb{E}(X, Y)^{(t)}$.

In section 13, we use the twisting spectral sequence from [T2]. In our case, it is proved in [T2] that the spectral sequence collapses at the second page. As a consequence, we obtain that the $\mathcal{P}_{\mathbb{k}}$-graded algebras $\mathbb{E}\left(X^{(r)}, Y^{(s)}\right)$ can be easily computed (up to a filtration) from $\mathbb{E}\left(X^{(r-s)}, Y\right)$.

To finish the computation, we need the filtrations on $\mathbb{E}\left(X^{(r)}, Y^{(s)}\right)$ to be trivial. This is the purpose of section 14, which is of independent interest. We prove that for some filtered $\mathcal{P}_{\mathbb{k}}$-graded algebras $A$ with prescribed $\operatorname{Gr} A$, the filtration must split. The results are stated in section 15 . 


\section{DÉCAlages}

Let $A=\left\{A_{0, d}\right\}_{d \geq 0}$ be a $\mathcal{P}_{\mathbb{k}}$-algebra. Recall the external product:

$$
\mathbb{E}^{i}\left(F, A_{0, d}\right) \otimes \mathbb{E}^{j}\left(G, A_{0, e}\right) \rightarrow \mathbb{E}^{i+j}\left(F \otimes G, A_{0, d+e}\right)
$$

induced by tensor products and the multiplication of $A$. The following décalage formula was first obtained, by other means, in [C2, Prop 2.6].

Proposition 12.1. Let $\mathbb{k}$ be a field of prime characteristic $p$, let $F \in \mathcal{P}_{\mathbb{k}}$, and let $t$ be a nonnegative integer. For all integer $i$, there are isomorphisms of strict polynomial functor $\$$, natural in $F$ :

$$
\begin{aligned}
& \mathbb{E}^{i}\left(F, S^{d}\right)^{(t)} \simeq \mathbb{E}^{i}\left(F^{(t)}, S^{d p^{t}}\right), \\
& \mathbb{E}^{i}\left(F, \Lambda^{d}\right)^{(t)} \simeq \mathbb{E}^{i+\left(p^{t}-1\right) d}\left(F^{(t)}, \Lambda^{d p^{t}}\right), \\
& \mathbb{E}^{i}\left(F, \Gamma^{d}\right)^{(t)} \simeq \mathbb{E}^{i+2\left(p^{t}-1\right) d}\left(F^{(t)}, \Gamma^{d p^{t}}\right) .
\end{aligned}
$$

Moreover, if $Y=S, \Lambda$ or $\Gamma$, the external product

$$
\mathbb{E}^{*}\left(F, Y^{d}\right)^{(t)} \otimes \mathbb{E}^{*}\left(G, Y^{e}\right)^{(t)} \rightarrow \mathbb{E}^{*}\left(F \otimes G, Y^{d+e}\right)^{(t)}
$$

identifies through the isomorphism above with the external product:

$$
\mathbb{E}^{*}\left(F^{(t)}, Y^{d p^{t}}\right) \otimes \mathbb{E}^{*}\left(G^{(t)}, Y^{e p^{t}}\right) \rightarrow \mathbb{E}^{*}\left(F^{(t)} \otimes G^{(t)}, Y^{d p^{t}+e p^{t}}\right) .
$$

Proof. We will prove that the isomorphisms actually hold at the level of chain complexes. Since there are no extension groups between homogeneous functors of different weights, we can assume that $F$ is homogeneous of weight $d$. Let us recall from [T2, Lemmas 2.2 and 2.3] an elementary computation in $\mathcal{P}_{\mathbb{k}}$. If $\mu=\left(\mu_{1}, \ldots, \mu_{n}\right)$ is a tuple of positive integers, we denote by $S^{\mu}$ the tensor product $S^{\mu_{1}} \otimes \cdots \otimes S^{\mu_{n}}$, and by $\alpha \mu$ the tuple $\alpha \mu:=\left(\alpha \mu_{1}, \ldots, \alpha \mu_{n}\right)$. For all $G \in \mathcal{P}_{\mathbb{k}}$, there are isomorphisms (the first one is induced by precomposition by $I^{(t)}$, the second one by the canonical inclusion $\left.S^{\mu(t)} \hookrightarrow S^{p^{t} \mu}\right)$ :

$$
\begin{aligned}
& \mathbb{H}\left(F, S^{\mu}\right)^{(t)} \simeq \mathbb{H}\left(F^{(t)}, S^{\mu(t)}\right), \\
& \mathbb{H}\left(F^{(t)}, S^{\mu(t)}\right) \simeq \mathbb{H}\left(F^{(t)}, S^{p^{t} \mu}\right), \\
& \mathbb{H}\left(F^{(t)}, S^{\lambda}\right) \simeq 0 \text { if } \lambda \text { is not of the form } p^{t} \mu .
\end{aligned}
$$

Since $\mathbb{E}^{*}\left(F, S^{d}\right)^{(t)}=\mathbb{H}\left(F, S^{d}\right)^{(t)}$, the composition of the isomorphisms (i) and (ii) provides the required isomorphism for the case of $S^{d}$. The compatibility with external products is straightforward.

Now we turn to the case of $\mathbb{E}^{*}\left(F, \Lambda^{d}\right)^{(t)}$. We use bar constructions of symmetric algebras in the same fashion as in the proof of proposition 7.1 . There a quasi-isomorphism of dg- $\mathcal{P}_{\mathbb{k}}$-algebras $\Lambda[1] \hookrightarrow \bar{B} S$, which becomes after regrading a quasi-isomorphism $\Lambda \hookrightarrow{ }^{t} \mathcal{R}_{1}(\bar{B} S)$. The homogeneous part of weight $d$ of ${ }^{\mathrm{t}} \mathcal{R}_{1}(\bar{B} S)$ is a coresolution of $\Lambda^{d}$ by symmetric powers, which we denote by ${ }^{\mathrm{t}} \mathcal{R}_{1} I_{d}$. So $\mathbb{E}^{i}\left(F^{(t)}, \Lambda^{d p^{t}}\right)$ is the $(-i)$-th homology group of the

\footnotetext{
${ }^{6}$ In the statement, we take the convention that $\mathbb{E}^{i}(F, G)=0$ for $i<0$. To emphasize the analogy between the three cases, we have also written $\mathbb{E}^{i}\left(F, S^{d}\right)$ although these extension groups actually reduce to $\mathbb{H}\left(F, S^{d}\right)$ since symmetric powers are injective.
} 
complex $\mathbb{H}\left(F^{(t)},{ }^{\mathrm{t}} \mathcal{R}_{1} I_{d p^{t}}\right)$. We consider the morphism of complexes defined as the composition

$$
\begin{aligned}
\mathbb{H}\left(F,{ }^{\mathrm{t}} \mathcal{R}_{p^{t}} I_{d}\right){ }^{(t)} & \left.\simeq \mathbb{H}\left(F^{(t)},{ }^{\mathrm{t}} \mathcal{R}_{p^{t}} I_{d}\right){ }^{(t)}\right) \\
& =\mathbb{H}\left(F^{(t)},{ }^{\mathrm{t}} \mathcal{R}_{1}\left(I_{d}^{(t)}\right)\right) \rightarrow \mathbb{H}\left(F^{(t)},{ }^{\mathrm{t}} \mathcal{R}_{1} I_{d p^{t}}\right) .
\end{aligned}
$$

where the first isomorphism is the isomorphism $(i)$, the equality comes from the equality $\mathcal{R}_{1}\left(\bar{B} S^{(t)}\right)=\left(\mathcal{R}_{p^{t}}(\bar{B} S)\right)^{(t)}$, and the last morphism is induced by the morphism of graded $\mathcal{P}_{\mathbb{k}}$-algebras $S^{(t)} \hookrightarrow S$ (which maps $S^{d(t)}$ into $S^{d p^{t}}$ ), which induces a morphism $\bar{B} S^{(t)} \hookrightarrow \bar{B} S$, hence a morphism ${ }^{\mathrm{t}} \mathcal{R}_{1}\left(I_{d}^{(t)}\right) \rightarrow{ }^{\mathrm{t}} \mathcal{R}_{1} I_{d p^{t}}$. We claim that the last map in the composition $(i v)$ is an isomorphism of complexes. Indeed, the objects of the complex ${ }^{\mathrm{t}} \mathcal{R}_{1} I_{d p^{t}}$ are symmetric tensors $S^{\lambda}$. If $\lambda=p^{t} \mu$, then there is a summand $S^{\mu(t)}$ of $\left({ }^{\mathrm{t}} \mathcal{R}_{-1} I_{d}\right){ }^{(t)}$ such that the restriction of the map ${ }^{\mathrm{t}} \mathcal{R}_{-1}\left(I_{d}^{(t)}\right) \rightarrow{ }^{\mathrm{t}} \mathcal{R}_{-1} I_{d p^{t}}$ to $S^{\mu(t)}$ is the canonical inclusion $S^{\mu(t)} \hookrightarrow S^{p^{t} \mu}$. This canonical inclusion induces an isomorphism after applying $\mathbb{H}\left(F^{(t)},-\right)$, by $(v)$. If $\lambda$ is not of the form $p^{t} \mu$, then $\mathbb{H}\left(F^{(t)}, S^{\lambda}\right) \simeq 0$ by (iii). Hence the composition (iv) is an isomorphism of complexes. Taking the $(-i)$-th homology group, we obtain an isomorphism

$$
\mathbb{E}^{i}\left(F^{(t)}, \Lambda^{d p^{t}}\right) \simeq \mathbb{E}^{i-d\left(p^{t}-1\right)}\left(F, \Lambda^{d}\right)^{(t)} .
$$

It remains to check the compatibility with external products. This is a straightforward check at the level of cochain complexes, similar to the identification of the convolution product in the proof proposition 7.1, The case of $\mathbb{E}^{*}\left(F, \Gamma^{d}\right)$ is similar.

If $C$ is a $\mathcal{P}_{\mathbb{R}^{-}}$-coalgebra, and $Y$ is a classical exponential functor, the convolution product of $\mathbb{E}\left(C, Y^{(t)}\right)$ is obtained by combining the external product and the map induced by the comultiplication of $C$. So proposition $12.1 \mathrm{im}-$ plies the following result, which we formulate using the regrading functor $\mathcal{R}_{\alpha}$ from section 6 .

Corollary 12.2. Let $C$ be a $\mathcal{P}_{\mathbb{k}}$-coalgebra and let $Y$ be a classical exponential functor. For all $t \geq 0$, there is an isomorphism $\mathcal{P}_{\mathbb{k}}$-graded algebras:

$$
\left(\mathcal{R}_{\alpha(Y)} \mathbb{E}(C, Y)\right)^{(t)} \simeq \mathbb{E}\left(C^{(t)}, Y\right),
$$

with $\alpha(S)=0, \alpha(\Lambda)=p^{t}-1$ and $\alpha(\Gamma)=2\left(p^{t}-1\right)$.

For the convenience of the reader, we write out explicitly two cases of application of corollary 12.2 ,

Example 12.3. (1) The divided powers $\Gamma^{d}$ are projective strict polynomial functors, and the Yoneda lemma yields an isomorphism of $\mathcal{P}_{\mathbb{k}}$-algebras:

$$
\mathbb{E}(\Gamma, \Lambda)=\mathbb{H}(\Gamma, \Lambda) \simeq \Lambda .
$$

Let $\Lambda\langle i\rangle^{(t)}$ denotes the $\mathcal{P}_{\mathbb{k}}$-graded algebra with $\Lambda^{d(t)}$ in cohomological degree $i$ and weight $d p^{t}$ (hence $\Lambda\langle i\rangle^{(t)}=\Lambda[-i]^{(t)}$ ). Corollary 12.2 implies:

$$
\mathbb{E}\left(\Gamma^{(t)}, \Lambda\right) \simeq \Lambda\left\langle p^{t}-1\right\rangle^{(t)} .
$$


(2) Assume that $p=2$. Let $I^{(k)}\langle i\rangle$ denote a copy of the $k$-th Frobenius twist functor, placed in cohomological degree $i$ (hence $I^{(k)}\langle i\rangle=I^{(k)}[-i]$ ). We computed $E(S, \Lambda)$ in theorem 10.17, so corollary 12.2 implies:

$$
\mathbb{E}\left(S^{(t)}, \Lambda\right) \simeq \Gamma\left(\bigoplus_{k \geq 0} I^{(k+t)}\left\langle p^{k+t}-1\right\rangle\right) .
$$

\section{The twisting spectral sequence and Troesch complexes}

Let $r, s$ be nonnegative integers, and assume that $t:=r-s$ is nonnegative. In this section we explain how to recover, up to a filtration, the $\mathcal{P}_{\mathbb{k}}$-graded algebra $\mathbb{E}\left(X^{(r)}, Y^{(s)}\right)$ from the $\mathcal{P}_{\mathbb{k}}$-graded algebra $\mathbb{E}\left(X^{(t)}, Y\right)$. This is a simple application of the results proved in [T2], but for the reader's convenience we recall here the results we need for our computation.

13.1. Parameterization by graded vector spaces. Let $V$ be a finite dimensional graded vector space. The parameterized functor $F_{V}: W \mapsto$ $F(V \otimes W)$ actually carries a grading, defined in the following way. Let the multiplicative group $\mathbb{G}_{m}$ act rationally on a homogeneous vector $v \in V$ of degree $i$ by the formula $\lambda \cdot v:=\lambda^{i} v$. Since $F$ is a strict polynomial functor, this induces a rational action of $\mathbb{G}_{m}$ on $F_{V}(W)$ for all $W \in \mathcal{V}_{\mathbb{k}}$. Thus, $F_{V}$ splits as a direct sum $F_{V}=\bigoplus_{i \in \mathbb{Z}}\left(F_{V}\right)_{i}$, where $\left(F_{V}\right)_{i}(W)$ is the subspace of weight $i$ of $F_{V}(W)$ under the action of $\mathbb{G}_{m}$.

Thus, parameterization by a graded vector space $V$ defines a weightpreserving functor $\left(\mathcal{P}_{\mathbb{k}}^{*}\right.$ is the category of graded strict polynomial functors):

$$
\begin{gathered}
\mathcal{P}_{\mathbb{k}} \rightarrow \mathcal{P}_{\mathbb{k}}^{*} \\
F \mapsto F_{V} .
\end{gathered}
$$

Example 13.1. If $F$ is a symmetric power, an exterior power, a divided power or a tensor product of these, the notion of degree defined on $F_{V}(W)$ coincides with the usual definition. For example, if $V$ has a homogeneous basis formed by $e_{-1}$ of degree -1 and $e_{4}$ of degree 4 , and if $w_{i}$ denote elements of $W$, the element $\left(e_{-1} \otimes w_{1}\right)\left(e_{4} \otimes w_{2}\right) \otimes\left(e_{-1} \otimes w_{3}\right)$ is an element of degree $-1+4-1=2$ of $\left(S^{2} \otimes S^{1}\right)_{V}(W)$.

The following example will also be important for our computations.

Example 13.2. If $F=I^{(r)}$ and $V=\oplus V_{i}$ then $\left(I^{(r)}\right)_{V}=\oplus V_{i}^{(r)} \otimes I^{(r)}$, where the summand $V_{i}^{(r)} \otimes I^{(r)}$ is homogeneous of degree $p^{r} i$. In particular, the functors $\left(I_{V}\right)^{(r)}$ and $\left(I^{(r)}\right)_{V}$ do not bear the same gradings, although they are isomorphic in an ungraded way.

For our purposes, we need to define parameterization of $\mathcal{P}_{\mathbb{k}}$-graded algebras by graded vector spaces. Let $A$ be a $\mathcal{P}_{\mathbb{k}}$-graded algebra and denote by $A_{i, d}$ the homogeneous part of $A$ of degree $i$ and weight $d$. The parameterized functor $\left(A_{i, d}\right)_{V}$ has a weight and a bigrading. To be more specific, the first partial degree is the degree $i$ coming from the grading of $A$, and the second partial degree is induced by the grading of $V$. To get rid of bidegrees, we define the degree on $\left(A_{i, d}\right)_{V}$ as the total degree associated to the bidegree. In this way, parameterization by a graded vector space $V$ defines a functor:

$$
\begin{array}{ccc}
\left\{\mathcal{P}_{\mathbb{k}}-\text { g-alg }\right\} & \rightarrow & \left\{\mathcal{P}_{\mathbb{k}}-\text { g-alg }\right\} \\
A & \mapsto & A_{V}
\end{array}
$$


Example 13.3. Let $V$ be a graded vector space with homogeneous basis $\left(e_{2}, e_{4}\right)$ with $e_{i}$ of degree $i$. Then $S[16]_{V}=S[18] \otimes S[20]$.

13.2. The twisting spectral sequence. Let us denote by $E_{s}$ the graded vector space $\operatorname{Ext}_{\mathcal{P}_{\mathbb{k}}}^{*}\left(I^{(s)}, I^{(s)}\right)$. It is one dimensional in cohomological degrees $2 i$, for $0 \leq i<p^{s}$ and zero everywhere else, see e.g. [FS, Thm 4.5] or [T2, Cor 4.7]. If $G \in \mathcal{P}_{\mathbb{k}}$, the parameterized functor $G_{E_{s}}$ inherits from $E_{s}$ a cohomological grading, as explained in section 13.1. We denote by $\left(G_{E_{s}}\right)^{j}$ its homogeneous part of cohomological 7 degree $j$.

Recall from [T2, Thm 7.1] that for strict polynomial functors $F, G$ with finite dimensional values and for $s \geq 0$, there is a 'twisting spectral sequence', natural with respect to $F$ and $G$ :

$$
E^{i, j}(F, G, s):=\operatorname{Ext}_{\mathcal{P}_{\mathrm{k}}}^{i}\left(F,\left(G_{E_{s}}\right)^{j}\right) \Longrightarrow \operatorname{Ext}_{\mathcal{P}_{\mathbb{k}}}^{i+j}\left(F^{(s)}, G^{(s)}\right) .
$$

Let us put the parameterized strict polynomial functor $G_{V^{(s)}}$ instead of $G$ in the spectral sequence above. Since we have

$$
\left(G_{V^{(s)}}\right)^{(s)}=\left(G^{(s)}\right)_{V} \text { and }\left(G_{V^{(s)}}\right)_{E_{s}}=G_{V^{(s)} \otimes E_{s}},
$$

the spectral sequence now takes the form:

$$
E^{i, j}\left(F, G_{V^{(s)}}, s\right)=\left(\mathbb{E}^{i}(F, G)_{E_{s}}\right)^{(s) j} \Longrightarrow \mathbb{E}^{i+j}\left(F^{(s)}, G^{(s)}\right),
$$

where for all $i \geq 0,\left(\mathbb{E}^{i}(F, G)_{E_{s}}\right)^{(s) j}$ denotes the homogeneous part of cohomological degree $j$ of the graded strict polynomial functor $\left(\mathbb{E}^{i}(F, G)_{E_{s}}\right)^{(s)}$.

If $G$ is a symmetric power, an exterior power, a divided power or a tensor product of these, it is proved in [T2, Thm 8.11] that the spectral sequence collapses at the second page.

Finally, it is proved in [T2, Thm 7.1] that the twisting spectral is compatible with tensor products and natural with respect to $F$ and $G$. Thus, if $X$ and $Y$ are classical exponential functors and if $t \geq 0$, we can retrieve (up to a filtration) the convolution product of the abutment $\mathbb{E}\left(X^{(s+t)}, Y^{(s)}\right)$ from the convolution product of the second page $\left(\mathbb{E}\left(X^{(t)}, Y\right)_{E_{s}}\right)^{(s)}$. The following statement sums up our discussion.

Theorem 13.4. Let $\mathbb{k}$ be a field of positive characteristic, let $X, Y$ be classical exponential functors, and let $t$ and $s$ be nonnegative integers. Then $\mathbb{E}\left(X^{(t+s)}, Y^{(s)}\right)$ is a filtered $\mathcal{P}_{\mathbb{k}}$-graded algebra and there is an isomorphism of $\mathcal{P}_{\mathbb{k}}$-graded algebras:

$$
\left(\mathbb{E}\left(X^{(t)}, Y\right)_{E_{s}}\right)^{(s)} \simeq \operatorname{Gr}\left(\mathbb{E}\left(X^{(t+s)}, Y^{(s)}\right)\right) .
$$

The algebra appearing on the left hand side of the isomorphism of theorem 13.4 is easy to compute with the help of corollary 12.2 .

Example 13.5. (1) Let $I^{(k)}\langle i\rangle$ denote a copy of the $k$-th Frobenius twist, with cohomological degree $i$ (hence $I^{(k)}\langle i\rangle=I^{(k)}[-i]$ ). There is, up to a filtration, an isomorphism of $\mathcal{P}_{\mathbb{k}^{k}}$-graded algebras:

$$
\mathbb{E}\left(\Gamma^{(t+s)}, \Lambda^{(s)}\right) \simeq \Lambda\left(\bigoplus_{0 \leq i<p^{s}} I^{(t+s)}\left\langle(2 i+1) p^{t}-1\right\rangle\right) .
$$

\footnotetext{
${ }^{7}$ As usual, we can convert cohomological degrees into homological degrees by the formula $M^{i}=M_{-i}$.
} 
(2) Assume that $p=2$. There is, up to a filtration, an isomorphism of $\mathcal{P}_{\mathbb{k}}$-graded algebras:

$$
\mathbb{E}\left(S^{(t+s)}, \Lambda^{(s)}\right) \simeq \Gamma\left(\bigoplus_{0 \leq i<p^{s}} \bigoplus_{k \geq 0} I^{(k+t+s)}\left\langle(2 i+1) p^{k+t}-1\right\rangle\right) .
$$

\section{Splitting Filtrations}

This section is of independent interest: we prove general splitting results for filtrations of $\mathcal{P}_{\mathbb{k}}$-graded algebras. We will use these results in section 15 to prove that the filtrations appearing in theorem 13.4 split.

14.1. General facts on filtrations. We begin with elementary observations about filtered functors.

Lemma 14.1. Let $\mathbb{k}$ be a field and let $F$ be a filtered strict polynomial functor over $\mathbb{k}$ with finite dimensional values.

(i) The filtration of $F$ has finite length.

(ii) If $\operatorname{Ext}_{\mathcal{P}_{\mathrm{k}}}^{1}(\mathrm{Gr} F, \mathrm{Gr} F)=0$, there is an isomorphism $F \simeq \mathrm{Gr} F$.

Proof. To prove (i), observe that a filtration of $F$ is equivalent to a filtration of the $S\left(\mathbb{k}^{d}, d\right)$-module $F\left(\mathbb{k}^{d}\right)$ where $d$ is the strict polynomial degree of $F$. Hence the filtration is finite for dimension reasons. Let us prove (ii). Let $F_{1} \subset F_{2} \subset \cdots \subset F_{n}=F$ be the filtration of $F$. If $\operatorname{Ext}_{\mathcal{P}_{\mathrm{k}}}^{1}(\mathrm{Gr} F, \operatorname{Gr} F)$ equals zero, then $\operatorname{Ext}_{\mathcal{P}_{\mathrm{k}}}^{1}\left(F_{1}, F_{2} / F_{1}\right)$ equals zero. Hence the extension $F_{1} \hookrightarrow F_{2} \rightarrow$ $F_{2} / F_{1}$ splits, that is $F_{2} \simeq F_{1} \oplus F_{2} / F_{1}$. In this way we build inductively an isomorphism $F \simeq \mathrm{Gr} F$.

Without further indication, filtrations of $\mathcal{P}_{\mathbb{k}}$-graded algebras will always mean bounded filtrations (that is, on each homogeneous component $A_{i, d}$ of the $\mathcal{P}_{\mathbb{k}}$-graded algebra $A$, the filtration has finite length). For example, filtrations of exponential functors are always bounded (since the $E_{i, d}$ take finite dimensional values, this follows from lemma 14.1). Also, filtered $\mathcal{P}_{k^{-}}$ graded algebras appearing as abutments of first quadrant spectral sequences like the twisting spectral sequence from section 13 have bounded filtrations. In our study of filtered $\mathcal{P}_{\mathbb{k}}$-graded algebras, we shall use $(1, \epsilon)$-commutativity defined in section 4.2. The following lemma is an easy check.

Lemma 14.2. Let $A$ be a filtered $\mathcal{P}_{\mathbb{k}}$-graded algebra. If $A$ is $(1, \epsilon)$ commutative, so is $\operatorname{Gr} A$.

Finally, we observe that the exponential property behaves well with filtrations of algebras.

Lemma 14.3. Let $\mathbb{k}$ be a field and let $A$ be a filtered $\mathcal{P}_{\mathbb{k}}$-graded algebra. If $\operatorname{Gr} A$ is a graded exponential functor, then so is $A$.

Proof. Consider $A(V) \otimes A(W)$ as a weighted graded $\mathbb{k}$-module with the tensor product filtration

$$
F^{i}(A(V) \otimes A(W))=\sum_{k+\ell=i} F^{k} A(V) \otimes F^{\ell} A(W) .
$$

The multiplication induces a map of weighted graded $\mathbb{k}$-modules $A(V) \otimes$ $A(W) \stackrel{\mathrm{m}}{\rightarrow} A(V \oplus W)$ compatible with the filtrations. Since $\operatorname{Gr} A$ is exponential, the associated graded map Grm is an isomorphism on each homogeneous part of degree $i$ and weight $k$. The filtrations of the homogeneous 
part of degree $i$ and weight $d$ has finite length. So by iterated uses of the five lemma, the map $m:(A(V) \otimes A(W))_{i, k} \rightarrow A(V \oplus W)_{i, k}$ is also an isomorphism. Thus $A$ is exponential.

\subsection{Splitting results.}

\subsubsection{The odd characteristic case.}

Lemma 14.4. Let $\mathbb{k}$ be a field of odd characteristic. Let $m, n$ be positive integers, let $\lambda$ and $\alpha$ be $m$-tuples of nonnegative integers and let $\mu$ and $\beta$ are $n$-tuples of nonnegative integers. Let $G$ be a functor of the form $G=\left(\bigotimes_{i=1}^{m} S^{\lambda_{i}\left(\alpha_{i}\right)}\right) \otimes\left(\bigotimes_{i=1}^{n} \Lambda^{\mu_{i}\left(\beta_{i}\right)}\right)$. Then $\operatorname{Ext}_{\mathcal{P}_{\mathbf{k}}}^{1}(G, G)=0$.

Proof. By iterated uses of lemma 5.7 (or use [FFSS, Cor 1.8]), it suffices to check that $\operatorname{Ext}^{1}(X, Y)=0$ if $X$ and $Y$ are of the form $S^{\ell(r)}$ or $\Lambda^{m(s)}$.

We first check that it is true if the functors are not twisted: $\operatorname{Ext}^{1}\left(S^{d}, S^{d}\right)=$ $\operatorname{Ext}^{1}\left(\Lambda^{d}, S^{d}\right)=0$ by injectivity of $S^{d}, \operatorname{Ext}^{1}\left(\Lambda^{d}, \Lambda^{d}\right)=0$ by remark 7.6, and $\operatorname{Ext}^{1}\left(S^{d}, \Lambda^{d}\right)=0$ by theorem 10.16. By proposition 12.1, Ext ${ }^{1}$ also vanish if one of the two functors $X, Y$ is twisted (indeed, the Ext-degree is shifted by an even integer since $p$ is odd). Finally theorem 13.4 shows the vanishing of $\operatorname{Ext}^{1}(X, Y)$ in the general case (indeed, if $A$ is a negatively graded $\mathcal{P}_{\mathbb{k}}$-algebra which is zero in degree -1 , then so is $A_{E_{s}}$ ).

Proposition 14.5 (Splitting result I). Let $\mathbb{k}$ be a field of odd characteristic, and let $A$ be a filtered $\mathcal{P}_{\mathbb{k}}$-graded algebra. Assume that $A$ is graded commutative and that $\operatorname{Gr} A$ has one of the following form:

$$
\text { (i) } S(F) \otimes \Lambda(G), \quad(i i) \Gamma(F) \otimes \Lambda(G),
$$

where $F$ and $G$ are graded strict polynomial functors which are additive. Then there is an isomorphism of $\mathcal{P}_{\mathbb{k}}$-graded algebras $A \simeq \operatorname{Gr} A$.

Proof. The proof relies essentially on lemma 14.4 and the use of universal properties of $\operatorname{Gr} A$. We prove case (ii), which is slightly more difficult.

Step 1: splitting without products. For all $i, d$, the extension group $\operatorname{Ext}_{\mathcal{P}_{\mathrm{k}}}^{1}\left(\operatorname{Gr} A_{i, d}, \operatorname{Gr} A_{i, d}\right)$ equals $\operatorname{Ext}_{\mathcal{P}_{\mathbb{k}}}^{1}\left(\operatorname{Gr} A_{i, d}^{\sharp}, \operatorname{Gr} A_{i, d}^{\sharp}\right)$ by lemma 2.3, which equals zero by lemma 14.4. So we get an isomorphism of graded strict polynomial functors $A \simeq \operatorname{Gr} A$, whence a surjective map: $\phi: A \rightarrow F \oplus G$. There is an extra grading on $\operatorname{Gr} A$, namely the filtration degree (that is $G r_{k} A$ is the homogeneous part of $\operatorname{Gr} A$ of filtration degree $k$ ). If we define a filtration on $\operatorname{Gr} A$ (hence on $F \oplus G$ ) by $\mathcal{F}_{k} \operatorname{Gr} A=\bigoplus_{i \leq k} \operatorname{Gr}_{i} A$, then $\phi$ preserves the filtrations, and $\operatorname{Gr} \phi$ equals the canonical subjection $\operatorname{Gr} A \rightarrow F \oplus G$.

Step 2: coalgebra structures and universal property. Since $F$ and $G$ are additive, $\operatorname{Gr} A$ is a graded exponential functor. Thus, by lemma 14.3 , $A$ is a graded exponential functor. In particular, $A$ also has a coalgebra structure. Since the filtration of $A$ is natural and compatible with products, $A$ is a filtered coalgebra, and $\operatorname{Gr}(A)=\Gamma(F) \otimes^{\epsilon} \Lambda(G)$ as a coalgebra.

Now $\operatorname{Gr} A$ is a graded commutative algebra. Hence $\Gamma(F)$ and $\Lambda(G)$ are graded commutative. Hence $F$ (resp. $G$ ) is concentrated in even (resp. odd) degrees. Thus, $\Gamma(F) \otimes \Lambda(G)$ is the universal cofree graded commutative coalgebra on $F \oplus G$. Thus, by the universal property of the cofree algebra (see e.g. [LV, Chap. 1]) $\phi$ induces a map $\psi: A \rightarrow \Gamma(F) \otimes \Lambda(G)$. 
Step 3: conclusion Let us consider the trivial filtration on $\Gamma(F) \otimes$ $\Lambda(G)$ induced by the filtration $\mathcal{F}_{k}(F \otimes G)$ of $F \otimes G$ from Step 1. Then $\psi$ preserves the filtration and by construction $\operatorname{Gr} \psi$ equals the identity map $\operatorname{Gr} A=\Gamma(F) \otimes \Lambda(G)$. In particular $\operatorname{Gr} \psi$ is an isomorphism. So $\psi$ is an isomorphism of coalgebras. Hence by lemma 5.4, $\psi$ is an isomorphism of algebras.

We also prove splitting results for $(1,1)$-commutative algebras. Recall the signed tensor product $\otimes^{1}$ defined in section 4.3.

Proposition 14.6 (Splitting result II). Let $\mathbb{k}$ be a field of odd characteristic, and let $A$ be a $(1,1)$-commutative filtered $\mathcal{P}_{\mathbb{k}}$-graded algebra over $\mathbb{k}$. Assume that $\operatorname{Gr} A$ has one of the following forms
(i) $S(F) \otimes^{1} \Lambda(G)$,
(ii) ${ }^{\mathrm{t}} S(F) \otimes{ }^{1} \Lambda(G)$,
(iii) $S(F) \otimes^{1 \mathrm{t}} \Lambda(G)$,
(vi) $\Gamma(F) \otimes 1 \Lambda(G)$,
(v) ${ }^{\mathrm{t}} \Gamma(F) \otimes{ }^{1} \Lambda(G)$,
(vi) $\Gamma(F) \otimes{ }^{1 \mathrm{t}} \Lambda(G)$,

where $F$ and $G$ are graded strict polynomial functors which are additive. Then there is an isomorphism of $\mathcal{P}_{\mathbb{k}}$-graded algebras $A \simeq \operatorname{Gr} A$.

Proof. The idea is to use the regrading functor $\mathcal{R}_{1}$ (and taking the opposite algebra of applying the functor ${ }^{\mathrm{t}}$ if needed) to go back to the graded commutative case. Let us treat the case where $\operatorname{Gr} A=S(F) \otimes \Lambda(G)$.

Since $A$ is $(1,1)$-commutative, so is $\operatorname{Gr} A$, hence so are $S(F)$ and $\Lambda(G)$ by lemma 4.13, Thus $F$ (resp. G) must be concentrated in odd (resp. even) degrees. Thus $\mathcal{R}_{1} A$ is graded commutative, with graded object

$$
\operatorname{Gr}\left(\mathcal{R}_{1} A\right)=\mathcal{R}_{1}(\operatorname{Gr} A)={ }^{\mathrm{t}} S\left(\mathcal{R}_{1} F\right) \otimes \Lambda\left(\mathcal{R}_{1} G\right) .
$$

Let us denote by $B^{\text {op }}$ the opposite algebra of an algebra $B$. Applying op and ${ }^{\mathrm{t}}$ (which do not alter graded commutativity) we get a graded commutative algebra $\left({ }^{\mathrm{t}} \mathcal{R}_{1} A\right)^{\mathrm{op}}$ with graded object $S\left(\mathcal{R}_{1} F\right) \otimes \Lambda\left(\mathcal{R}_{1} G\right)$. So by proposition 14.5. $\left({ }^{\mathrm{t}} \mathcal{R}_{1} A\right)^{\mathrm{op}}$ is isomorphic to $S\left(\mathcal{R}_{1} F\right) \otimes \Lambda\left(\mathcal{R}_{1} G\right)$. Applying op and $\mathcal{R}_{-1}$ to the isomorphism obtained, we get an isomorphism $A \simeq \operatorname{Gr} A$.

14.2.2. The characteristic two case. The following proposition is proved exactly in the same fashion as proposition 14.5.

Proposition 14.7 (Splitting result III). Let $\mathbb{k}$ be a field of characteristic 2, and let $A$ be a commutative filtered $\mathcal{P}_{\mathbb{k}}$-graded algebra. Assume that there is a graded strict polynomial functor $F$ which is additive, such that $\operatorname{Gr} A=S(F)$ or $\operatorname{Gr} A=\Gamma(F)$. Then $A \simeq \operatorname{Gr} A$ as $\mathcal{P}_{\mathbb{k}}$-graded algebras.

Proposition 14.7 will be sufficient to prove that the filtrations on the abutment of the twisting spectral sequence split in characteristic 2, except for the cases of $\mathbb{E}\left(\Lambda^{(t+s)}, S^{(s)}\right)$ and $\mathbb{E}\left(\Gamma^{(t+s)}, \Lambda^{(s)}\right)$. In these cases, the algebras are of exterior type. So we need a analogue of proposition 14.7 when $\operatorname{Gr} A=\Lambda(F)$. Two difficulties arise when we want to adapt the proof of proposition 14.5 to this case. First, there might be non-trivial extensions between certain twisted exterior powers in characteristic 2 (e.g. the extension $\left.\Lambda^{2} \hookrightarrow \Gamma^{2} \rightarrow \Lambda^{1(1)}\right)$. Second, exterior algebras still satisfy a universal property in characteristic 2 , but for strictly anticommutative algebras (recall that a $\mathbb{k}$-algebra is strictly anticommutative if for all $x \in A, x \cdot x=0$ ). So the best we can easily prove is the following statement. 
Proposition 14.8 (Splitting result IV). Let $\mathbb{k}$ be a field of characteristic 2, and let $A$ be a strictly anticommutative filtered $\mathcal{P}_{\mathbb{k}}$-graded algebra. Assume that $\operatorname{Gr} A$ equals $\Lambda(F)$, where $F$ is a graded strict polynomial functor of the following very specific form. There exists an integer $r$ such that $F$ is a direct sum of copies graded copies of $I^{(r)}$. Then $A \simeq \operatorname{Gr} A$ as $\mathcal{P}_{\mathbb{k}}$-graded algebras.

Proof. The proof that $\operatorname{Ext}_{\mathcal{P}_{\mathrm{k}}}^{1}\left(\operatorname{Gr} A_{i, d}, \operatorname{Gr} A_{i, d}\right)$ equals zero reduces, by iterated uses of lemma 5.7, to checking that for all $d \geq 0$, $\operatorname{Ext}_{\mathcal{P}_{k}}^{1}\left(\Lambda^{d(r)}, \Lambda^{d(r)}\right)$. But the latter fact follows from theorem 13.4 and the vanishing of $\mathbb{E}^{1}\left(\Lambda^{d}, \Lambda^{d}\right)$. So lemma 14.1 yields an isomorphism of bigraded strict polynomial functors $A \simeq \operatorname{Gr} A$. To get an isomorphism of algebras, we use the universal property of exterior algebras, exactly as in the third step of the proof of proposition 14.5, and we conclude the proof in the same fashion.

\section{Final RESUlts}

15.1. Statement of the results. We now describe the $\mathcal{P}_{\mathbb{k}}$-graded algebras $\mathbb{E}\left(X^{(r)}, Y^{(s)}\right)$, for all classical exponential functors $X, Y$. Duality yields an isomorphism of exponential functors $\mathbb{E}\left(X^{(r)}, Y^{(s)}\right) \simeq \mathbb{E}\left(Y^{\sharp(s)}, X^{\sharp(r)}\right)$, so we can restrict to the case $r \geq s$. Hence we write $r=t+s$, with $t \geq 0$. We recall the notations and conventions needed to read the statements.

(1) In the $\mathcal{P}_{\mathbb{k}}$-graded algebra:

$$
\mathbb{E}\left(X^{(t+s)}, Y^{(s)}\right)=\bigoplus_{i \geq 0, d \geq 0} \mathbb{E}^{i}\left(X^{d(s+t)}, Y^{p^{t} d(s)}\right)
$$

$\mathbb{E}^{i}\left(X^{d(s+t)}, Y^{p^{t} d(s)}\right)$ has cohomological degree $i$ and weight $d p^{s+t}$. The product is given by convolution, cf. definition 4.8 .

(2) The functor $I^{(r)}\langle h\rangle$ denotes a copy of the $r$-th Frobenius twist, placed in cohomological degree $h$ (and weight $p^{r}$ ). If $X=S, \Lambda$ or $\Gamma$, and $F$ is a graded functor, then $X(F)$ denotes the composite $X \circ F$, with the usual gradings. The tensor product $\otimes$ is the usual tensor product of graded algebras (i.e. weights do not bring any Koszul sign).

(3) In particular, in the theorems below, the functors $I^{(x+s+t)}\langle h\rangle$ appearing on the right hand side of the isomorphisms are direct summands of the functors $\mathbb{E}^{h}\left(X^{p^{x}(s+t)}, Y^{p^{x+t}(s)}\right)$.

We order the classical exponential functors as follows: $\Gamma<\Lambda<S$.

Theorem 15.1 (Pairs $(X, Y)$ with $X \leq Y)$. Let $\mathbb{k}$ be a field of positive characteristic $p$, and let $s, t$ be positive integers, and let $X$ be an arbitrary exponential functor. There are isomorphisms of $\mathcal{P}_{\mathbb{k}}$-graded algebras: 


$$
\begin{aligned}
& \mathbb{E}\left(X^{(t+s)}, S^{(s)}\right) \simeq X^{\sharp}\left(\bigoplus_{0 \leq i<p^{s}} I^{(t+s)}\left\langle 2 i p^{t}\right\rangle\right), \\
& \mathbb{E}\left(\Gamma^{(t+s)}, \Lambda^{(s)}\right) \simeq \Lambda\left(\bigoplus_{0 \leq i<p^{s}} I^{(t+s)}\left\langle(2 i+1) p^{t}-1\right\rangle\right), \\
& \mathbb{E}\left(\Lambda^{(t+s)}, \Lambda^{(s)}\right) \simeq \Gamma\left(\bigoplus_{0 \leq i<p^{s}} I^{(t+s)}\left\langle(2 i+1) p^{t}-1\right\rangle\right), \\
& \mathbb{E}\left(\Gamma^{(t+s)}, \Gamma^{(s)}\right) \simeq \Gamma\left(\bigoplus_{0 \leq i<p^{s}} I^{(t+s)}\left\langle(2 i+2) p^{t}-2\right\rangle\right) .
\end{aligned}
$$

Now we turn to the pairs $(X, Y)$, with $X>Y$. These algebras were not computed in [FFSS], where the authors suspected that there are 'no easy answer' for such pairs. Our approach explains why Ext-groups for these pairs are much more difficult to compute. Indeed, for all pairs $(X, Y)$, the $\mathcal{P}_{\mathbb{k}}$-algebra $\mathbb{E}\left(X^{(t+s)}, Y^{(t)}\right)$ can be deduced from $\mathbb{E}(X, Y)$. The latter are very easy to compute if $X \leq Y$ (they reduce to Hom-groups), but far from being trivial if $X>Y$ (they correspond to the homology of some Eilenberg Mac Lane spaces). The results of theorems 15.2, 15.3 and 15.4 do not coincide with the results of $[\mathrm{C} 2$, we compare them in section 15.3 .

Theorem 15.2 (Pairs $(X, Y)$ with $X>Y$ for $p=2$ ). Let $\mathbb{k}$ be a field of characteristic $p=2$, and let $s, t$ be positive integers. There are isomorphisms of $\mathcal{P}_{\mathbb{k}}$-graded algebras:

$$
\begin{aligned}
& \mathbb{E}\left(S^{(t+s)}, \Lambda^{(s)}\right) \simeq \Gamma\left(\bigoplus_{0 \leq i<p^{s}, 0 \leq k} I^{(k+t+s)}\left\langle(2 i+1) p^{k+t}-1\right\rangle\right), \\
& \mathbb{E}\left(\Lambda^{(t+s)}, \Gamma^{(s)}\right) \simeq \Gamma\left(\bigoplus_{0 \leq i<p^{s}, 0 \leq k} I^{(k+t+s)}\left\langle(2 i+2) p^{k+t}-p^{k}-1\right\rangle\right), \\
& \mathbb{E}\left(S^{(t+s)}, \Gamma^{(s)}\right) \simeq \Gamma\left(\bigoplus_{0 \leq i<p^{s}, 0 \leq k, 0 \leq \ell} I^{(k+\ell+t+s)}\left\langle(2 i+2) p^{k+\ell+t}-p^{k}-1\right\rangle\right) .
\end{aligned}
$$

The computation of $\mathbb{E}\left(S^{(t+s)}, \Lambda^{(s)}\right)$ and $\mathbb{E}\left(\Lambda^{(t+s)}, \Gamma^{(s)}\right)$ in odd characteristic brings special signs. Recall from section 4.3 that $A \otimes^{1} B$ denotes the 'signed tensor product' of two $\mathcal{P}_{\mathbb{k}^{k}}$-graded algebras, and ${ }^{\mathrm{t}} A$ denotes the weight twisted algebra associated to $A$ (defined in section $[6$ ). 
Theorem 15.3. Let $\mathbb{k}$ be a field of odd characteristic $p$, and let $s, t$ be positive integers. The $\mathcal{P}_{\mathbb{k}}$-graded algebra $\mathbb{E}\left(S^{(t+s)}, \Lambda^{(s)}\right)$ is isomorphic to:

$$
\begin{aligned}
& \Lambda\left(\bigoplus_{0 \leq i<p^{s}, 0 \leq k} I^{(k+t+s)}\left\langle(2 i+1) p^{k+t}-1\right\rangle\right) \\
& \quad \otimes^{1}{ }^{\mathrm{t}} \Gamma\left(\bigoplus_{0 \leq i<p^{s}, 0 \leq k} I^{(k+1+t+s)}\left\langle(2 i+1) p^{k+1+t}-2\right\rangle\right) .
\end{aligned}
$$

The $\mathcal{P}_{\mathbb{k}}$-graded algebra $\mathbb{E}\left(\Lambda^{(t+s)}, \Gamma^{(s)}\right)$ is isomorphic to:

$$
\begin{aligned}
& \Lambda\left(\bigoplus_{0 \leq i<p^{s}, 0 \leq k} V^{(k+t+s)}\left\langle(2 i+2) p^{k+t}-p^{k}-1\right\rangle\right) \\
& \quad \otimes^{1}{ }^{\mathrm{t}} \Gamma\left(\bigoplus_{0 \leq i<p^{s}, 0 \leq k} V^{(k+1+t+s)}\left\langle(2 i+2) p^{k+1+t}-p^{k+1}-2\right\rangle\right) .
\end{aligned}
$$

Theorem 15.4. Let $\mathbb{k}$ be a field of odd characteristic $p$, and let $s, t$ be positive integers. The $\mathcal{P}_{\mathbb{k}}$-graded algebra $\mathbb{E}\left(S^{(t+s)}, \Gamma^{(s)}\right)$ is isomorphic to the tensor product:

$$
\begin{aligned}
& \Gamma\left(\bigoplus_{0 \leq i<p^{s}, 0 \leq k} V^{(k+t+s)}\left\langle(2 i+2) p^{k+t}-2\right\rangle\right) \\
& \otimes \Lambda\left(\bigoplus_{0 \leq i<p^{s}, 0 \leq k, 0 \leq \ell} V^{(k+\ell+1+t+s)}\left\langle(2 i+2) p^{k+\ell+1+t}-2 p^{k}-1\right\rangle\right) \\
& \otimes \Gamma\left(\bigoplus_{0 \leq i<p^{s}, 0 \leq k, 0 \leq \ell} V^{(k+\ell+2+t+s)}\left\langle(2 i+2) p^{k+\ell+2+t}-2 p^{k+1}-2\right\rangle\right)
\end{aligned}
$$

15.2. Proof of theorems 15.1, 15.4. We proceed in several steps.

Step 1: Computation of $\mathbb{E}(X, Y)$. There are two cases. If $X \leq Y$, then $\mathbb{E}(X, Y)$ reduces to $\mathbb{H}(X, Y)$, which is easy to compute, cf. lemma 4.6. If $X>Y$, then $\mathbb{E}(X, Y)$ is rather complicated, and computed in theorems 10.16 and 10.17. In all cases, $\mathbb{E}(X, Y)$ is a symmetric, an exterior or a divided power algebra (or a tensor product of these) on some generators $I^{(b)}\langle a\rangle$.

Step 2: Computation of $\mathbb{E}\left(X^{(t)}, Y\right)$. By corollary 12.2, $\mathbb{E}\left(X^{(t)}, Y\right)$ is isomorphic to $\left(\mathcal{R}_{\alpha(Y)} \mathbb{E}(X, Y)\right)^{(t)}$. Under this isomorphism, each generator $I^{(b)}\langle a\rangle$ of $\mathbb{E}(X, Y)$ corresponds to a generator $I^{(b+t)}\left\langle a+p^{b} \alpha(Y)\right\rangle$ of $\mathbb{E}\left(X^{(t)}, Y\right)$, where $\alpha(S)=0, \alpha(\Lambda)=p^{t}-1$ and $\alpha(\Gamma)=2\left(p^{t}-1\right)$. Observe that in odd characteristic, $\alpha(Y)$ is even, so that no additional sign is introduced by the regrading functor $\mathcal{R}_{\alpha(Y)}$.

Step 3: Computation of $\mathbb{E}\left(X^{(t+s)}, Y^{(s)}\right)$, up to filtration. By theorem 13.4, $\mathbb{E}\left(X^{(t+s)}, Y^{(s)}\right)$ is, up to a filtration, an algebra of the same kind as $\mathbb{E}\left(X^{(t)}, Y\right)$, with more generators. To be more specific, each generator 
$I^{(b+t)}\left\langle a+p^{b} \alpha(Y)\right\rangle$ of $\mathbb{E}\left(X^{(t)}, Y\right)$ gives birth to a family of generators (indexed by an integer $i$, with $0 \leq i<p^{s}$ ):

$$
I^{(b+t+s)}\left\langle a+p^{b} \alpha\left(Y^{*}\right)+2 i p^{b+t}\right\rangle=I^{(b+t+s)}\left\langle\left(2 i p^{t}+\alpha(Y)\right) p^{b}+a\right\rangle .
$$

Step 4: Filtrations split. Finally, we have to prove that the filtrations involved are trivial. This follows directly from the splitting results of section 14. except in the cases of $\mathbb{E}\left(\Lambda^{(t+s)}, S^{(s)}\right)$ and $\mathbb{E}\left(\Gamma^{(t+s)}, \Lambda^{(s)}\right)$ when $\mathbb{k}$ has characteristic 2 . So let us assume that all the results but these two cases are proved. To prove the triviality of the filtrations of $\mathbb{E}\left(\Lambda^{(t+s)}, S^{(s)}\right)$ and $\mathbb{E}\left(\Gamma^{(t+s)}, \Lambda^{(s)}\right)$, we want to apply proposition 14.8. For this we need to check strict anticommutativity. So the following lemma finishes the proof.

Lemma 15.5. Let $\mathbb{k}$ be a field of characteristic 2. For all nonnegative integers $s, t$, the $\mathcal{P}_{\mathbb{k}}$-graded algebras $\mathbb{E}\left(\Lambda^{(t+s)}, S^{(s)}\right)$ and $\mathbb{E}\left(\Gamma^{(t+s)}, \Lambda^{(s)}\right)$ are strictly anticommutative (that is, for all $x, x \cdot x=0$ in these algebras).

Proof. Let us prove that $\mathbb{E}\left(\Gamma^{(t+s)}, \Lambda^{(s)}\right)$ is strictly anticommutative (the proof for $\mathbb{E}\left(\Lambda^{(t+s)}, S^{(s)}\right)$ is similar). Since $\mathbb{k}$ has characteristic 2 , we have an injective morphism of algebras $\alpha: \Lambda \hookrightarrow \Gamma$. It induces a morphism:

$$
\beta: \mathbb{E}\left(\Gamma^{(t+s)}, \Lambda^{(s)}\right) \rightarrow \mathbb{E}\left(\Lambda^{(t+s)}, \Lambda^{(s)}\right) .
$$

To prove that $\mathbb{E}\left(\Gamma^{(t+s)}, \Lambda^{(s)}\right)$ is strictly anticommutative, it suffices to prove that $\beta$ is injective. Indeed, we already know that $\mathbb{E}\left(\Lambda^{(t+s)}, \Lambda^{(s)}\right)$ is strictly anticommutative since it is a divided power algebra.

For all $d \geq 0$, we have a commutative square, where the vertical arrows are injections induced by the canonical map $\Lambda^{d} \hookrightarrow \otimes^{d}$ :

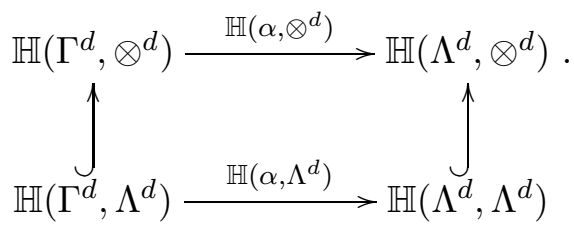

Now the map $\mathbb{H}\left(\alpha, \otimes^{d}\right)$ is an isomorphism (by lemma 5.5), so $\mathbb{H}\left(\alpha, \Lambda^{d}\right)$ is injective. Using proposition 12.1, we get an injection $\mathbb{H}\left(\Gamma^{d(t)}, \Lambda^{d p^{t}}\right) \hookrightarrow$ $\mathbb{H}\left(\Lambda^{d(t)}, \Lambda^{d p^{t}}\right)$. By theorem 13.4, $\operatorname{Gr}(\beta)$ equals the evaluation of this map on $E_{s} \otimes V^{(s)}$, hence it is injective. We deduce the injectivity of $\beta$.

\subsection{Comparison with [FFSS, C2].}

15.3.1. The case $X \leq Y$. In this case, the algebras

$$
\operatorname{Ext}_{\mathcal{P}_{\mathbb{k}}}^{*}\left(X^{*(s+t)}, Y^{*(s)}\right)=\mathbb{E}\left(X^{(s+t)}, Y^{(s)}\right)(\mathbb{k})
$$

were first computed in FFSS. Theorem 15.1 agrees with [FFSS, Thm 5.8]. For example, we assert that $\mathbb{E}(\Lambda, \Lambda)(\mathbb{k})$ is a divided power algebra on generators $g_{i} \in \operatorname{Ext}_{\mathcal{P}_{\mathrm{k}}}^{(2 i+1) p^{t}-1}\left(I^{(t+s)}, \Lambda^{p^{t}(s)}\right)$, for $0 \leq i<p^{s}$. This is exactly [FFSS, Thm 5.8(6)]. 
15.3.2. The case $X>Y$. The algebras with $X>Y$ were first computed in [C2]. Our computations differ from the results of [C2].

First, theorems 15.2 and 15.3 do not agree with [C2, Thm 3.2]. It is already explained in remark 4.7 why [C2, Thm 3.2] is false in characteristic 2. In odd characteristic, the two results agree as graded functors, but the descriptions of the products only agree up to signs. Unlike our result, C2, Thm 3.2] does not yield a $(1,1)$-commutative algebra. Indeed, in an algebra of the form $\Lambda(A) \otimes \Gamma(B)$, where $A$ is concentrated in even degrees and odd weights, and $B$ is concentrated in odd degrees and odd weights, if there are two non proportional elements $a_{1}, a_{2} \in A$, we can choose $b \in B$ and form the products $x=a_{1} \otimes b$ and $y=a_{2} \otimes \gamma_{2}(b)$, where $\gamma_{2}(b)=b \otimes b \in \Gamma^{2}(B)$. Then one has

$$
x \cdot y=\left(a_{1} \wedge a_{2}\right) \otimes b \gamma_{2}(b)=-\left(a_{2} \wedge a_{1}\right) \otimes \gamma_{2}(b) b=-y \cdot x .
$$

Since $x \cdot y \neq 0$, this contradicts $(1,1)$-commutativity.

Finally, theorem 15.4 does not agree with [C2, Cor 4.15]. To be more specific, the two results look the same, but in our computation, the resulting algebra has more generators. For example, theorem 15.4 asserts that $\mathbb{E}\left(S^{p}, \Gamma^{p}\right)$ is equal to $\Gamma^{p}\langle 0\rangle \oplus I^{(1)}\langle 2 p-2\rangle \oplus I^{(1)}\langle 2 p-3\rangle$, so the total dimension of $\mathbb{E}\left(S^{p}, \Gamma^{p}\right)(\mathbb{k})$ is 3 . On the other hand, [C2, Cor 4.15] predicts that this total dimension is 2 . So the following independent elementary computation argues in favor of our result.

Lemma 15.6. If $\mathbb{k}$ has characteristic $p$, then $\operatorname{Ext}_{\mathcal{P}_{\mathbb{k}}}^{i}\left(S^{p}, \Gamma^{p}\right)$ equals $\mathbb{k}$ if $i=$ $0,2 p-2$ or $2 p-3$, and is zero otherwise.

Proof. We need the following elementary facts. For $0 \leq k<p$, the functor $\Lambda^{k} \otimes S^{p-k}$ is injective as a direct summand of $\otimes^{k} \otimes S^{p-k}$, and $\Gamma^{k}$ is injective since it is isomorphic to $S^{k}$. Moreover:

$$
\begin{array}{ll}
\operatorname{Hom}_{\mathcal{P}_{\mathrm{k}}}\left(S^{p}, S^{p}\right)=\mathbb{k} & \text { with basis the identity map, } \\
\operatorname{Hom}_{\mathcal{P}_{\mathrm{k}}}\left(S^{p}, S^{1} \otimes S^{p-1}\right)=\mathbb{k} & \text { with basis the comultiplication, } \\
\operatorname{Hom}_{\mathcal{P}_{\mathrm{k}}}\left(S^{p}, \Lambda^{k} \otimes S^{p-k}\right)=0 & \text { for } k \geq 2 .
\end{array}
$$

To prove lemma 15.6, we cut the exact Koszul complexes:

$$
\begin{aligned}
& \Lambda^{p} \hookrightarrow \Lambda^{p-1} \otimes S^{1} \rightarrow \cdots \rightarrow \Lambda^{1} \otimes S^{p-1} \rightarrow S^{p} \\
& \Gamma^{p} \hookrightarrow \Gamma^{p-1} \otimes \Lambda^{1} \rightarrow \cdots \rightarrow \Gamma^{1} \otimes \Lambda^{p-1} \rightarrow \Lambda^{p}
\end{aligned}
$$

into short exact sequences and analyze the long $\operatorname{Ext}_{\mathcal{P}_{\mathfrak{k}}}^{*}\left(S^{p},-\right)$-exact sequence associated to them. We begin on the right with the first complex. Let $K$ be the kernel of the multiplication $S^{1} \otimes S^{p-1} \rightarrow S^{p}$. Since the composite of the comultiplication $S^{p} \rightarrow S^{1} \otimes S^{p-1}$ and the multiplication $S^{1} \otimes S^{p-1} \rightarrow S^{p}$ equals $p$ times the identity map (hence zero), we obtain that $\operatorname{Ext}^{i}\left(S^{p}, K\right) \simeq \mathbb{k}$ if $i=0,1$ and 0 otherwise. For the other short exact sequences except the one involving $\Gamma^{p}$, the terms $\operatorname{Ext}_{\mathcal{P}_{k}}^{*}\left(S^{p}, \Lambda^{k} \otimes S^{p-k}\right)$ and $\operatorname{Ext}_{\mathcal{P}_{k}}^{*}\left(S^{p}, \Lambda^{k} \otimes \Gamma^{p-k}\right)$ are trivial so the long exact sequence induces a shifting. Finally the last short exact sequence $\Gamma^{p} \hookrightarrow \Gamma^{1} \otimes \Gamma^{p-1} \rightarrow C$ induces a shifting and also creates a nonzero element in $\operatorname{Hom}_{\mathcal{P}_{\mathfrak{k}}}\left(S^{p}, \Gamma^{p}\right)$. 


\section{REFERENCES}

[A] K. Akin, Extensions of symmetric tensors by alternating tensors. J. Algebra 121 (1989), no. 2, 358-363.

[BMT] L. Breen, R. Mikhailov, A. Touzé, work in progress.

[Br] K.S. Brown, Cohomology of groups. Graduate Texts in Mathematics, 87. SpringerVerlag, New York-Berlin, 1982. $\mathrm{x}+306 \mathrm{pp}$.

[Car] H. Cartan, Séminaire Henri Cartan de l'École Normale supérieure, 1954/1955. Algèbres d'Eilenberg Mac Lane et homotopie, Secrétariat mathématique, 11 rue Pierre Curie, Paris, 1955 (French). Available online on www.numdam.org

[C1] M. Chałupnik, Extensions of strict polynomial functors. Ann. Sci. École Norm. Sup. (4) 38 (2005), no. 5, 773-792.

[C2] M. Chałupnik, Koszul duality and extensions of exponential functors. Adv. Math. 218 (2008), no. 3, 969-982.

[CPSvdK] E. Cline, B. Parshall, L. Scott, W. van der Kallen, Rational and generic cohomology. Invent. Math. 39 (1977), no. 2, 143-163.

[DP] A. Dold, D. Puppe, Homologie nicht-additiver Funktoren. Anwendungen. (German) Ann. Inst. Fourier Grenoble 11 (1961) 201-312.

[EML] S. Eilenberg, S. Mac Lane, On the groups of $H(\Pi, n)$. I. Ann. of Math. (2) 58, (1953). 55-106.

[Eis] D. Eisenbud, Commutative algebra. With a view toward algebraic geometry. Graduate Texts in Mathematics, 150. Springer-Verlag, New York, 1995. xvi+785 pp.

[FFSS] V. Franjou, E. Friedlander, A. Scorichenko, A. Suslin, General linear and functor cohomology over finite fields, Ann. of Math. (2) 150 (1999), no. 2, 663-728.

[FS] E. Friedlander, A. Suslin, Cohomology of finite group schemes over a field, Invent. Math. 127 (1997), 209-270.

[K] H. Krause, Koszul, Ringel, and Serre duality for strict polynomial functors, Compos. Math. 149 (2013), 996-1018.

[LV] J.L. Loday, B. Valette, Algebraic operads, Grundlehren der mathematischen Wissenschaften, Volume 346, Springer-Verlag (2012).

[ML] S. Mac Lane, Homology. Reprint of the 1975 edition. Classics in Mathematics. Springer-Verlag, Berlin, 1995. x+422 pp.

[P] V. Franjou, E. Friedlander, T. Pirashvili, L. Schwartz, Rational representations, the Steenrod algebra and functor homology. Panoramas et Synthèses [Panoramas and Syntheses], 16. Société Mathématique de France, Paris, 2003.

[S] J.-P. Serre, Cohomologie modulo 2 des complexes d'Eilenberg-MacLane. (French) Comment. Math. Helv. 27, (1953). 198-232.

[SFB] A. Suslin, E. Friedlander, C. Bendel, Infinitesimal 1-parameter subgroups and cohomology. J. Amer. Math. Soc. 10 (1997), no. 3, 693-728.

[T1] A. Touzé, Cohomology of classical algebraic groups from the functorial viewpoint, Adv. Math. 225 (2010), no. 1, 33-68.

[T2] A. Touzé, Troesch complexes and the twisting spectral sequence, Ann. Sci. École Norm. Sup. 45 (2012), no. 1, 53-99.

[W] C . Weibel, An introduction to homological algebra. Cambridge Studies in Advanced Mathematics, 38. Cambridge University Press, Cambridge, 1994. xiv+450 pp. 